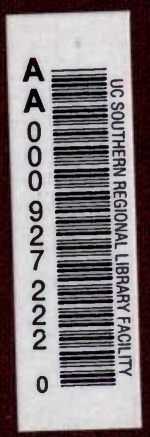




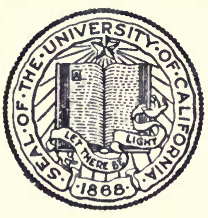

THE LIBRARY

OF

THE UNIVERSITY

OF CALIFORNIA

LOS ANGELES 
This book is DUE on the last date stamped below sem

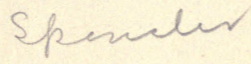

SOUTHERN BRANCH, UNIVERSITY OF CALIFORNIA, LIBRARY,

HOS ANGELES, CMEM 



\section{TROPICAL POLYPORES}

BY

WILliAM ALPHONSO MURRILL, A.M., PH.D.

ASSISTANT DIRECTOR OF THE NEW YORK BOTANICAL GARDEN

ASSOCIATE EDITOR OF NORTH AMERICAN FLORA

EDITOR OF MYCOLOGIA

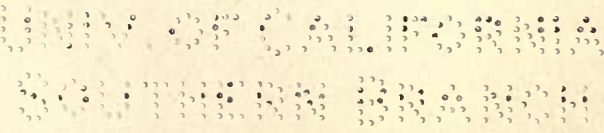

NEW YORK

PUBLISHED BY THE AUTHOR

1915

58587 


\section{Copyright, I9I5}

By William AlphoNSO MURRILl

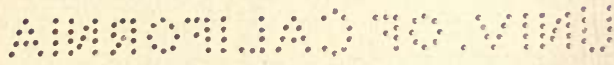

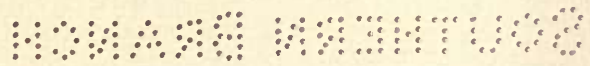




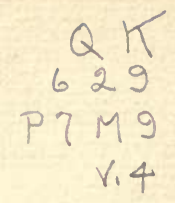

\section{PREFACE}

Polypores are tough or woody fungi found chiefly on wood in the form of brackets of various shapes and sizes, the fruiting surface being composed of tubes or furrows. Sometimes the walls of these tubes split with age and the hymenium appears spiny, resembling the hydnums; sometimes the furrows change with age to appear like gills. When the fruit-body is perennial, the tubes are often arranged in layers. The family may be

$\lambda$ divided into five groups, the resupinates, the annual poroid species, the volvate species, the perennial poroid species, and the agaric-like species. The resupinate species cannot be satisfactorily studied without the advantages of a large herbarium and are therefore omitted here, but some of the larger species of the other groups are comparatively easy.

Polypores as a class are very destructive to trees and timber. On the other hand, one species possesses medicinal properties, some of the encrusted species supply tinder, and several of the more juicy ones are excellent for food if collected when young. The only species recognized as poisonous is the medicinal one, Fomes Laricis, and it is so tough and bitter that no one would think of eating it.

Polypores are very easily collected and preserved and they largely retain their characters when dried, which makes them excellent objects for class study during the winter months. Many of them, also, remain in situ during the winter in perfect condition for collecting. As a group, they lend themselves remarkably well to studies in gross and minute anatomy, variation, adaptation, and injurious effects on trees and structural timbers.

North America may be conveniently divided into five regions: (I) eastern Canada and the northern United States southward to the southern boundaries of Virginia, Kentucky, Missouri, and Kansas, and westward to the western boundaries of Kansas, Nebraska, and the Dakotas; (2) the southern United States, iii 
including North Carolina, South Carolina, Tennessee, Arkansas, Oklahoma, Texas, Louisiana, Mississippi, Alabama, Georgia, and the northern portion of Florida; (3) the Rocky Mountain region, including the remainder of the western United States and Canada with the exception of states bordering on the Pacific Ocean; (4) the far West, including California, Oregon, Washington, British Columbia, and Alaska; and (5) tropical North America, including Mexico, Central America, southern Florida, the Bermudas, the West Indies, and all other islands between North America and South America with the exception of Trinidad.

In all these regions, there is an abundance of work still to be done before our knowledge of the polypores is complete, and it is believed that the publication of a series of books treating the species of each region separately will stimulate effort in this direction.

The terms here used to express the abundance of a species are "rare" or "occasional," "rather frequent," "frequent," "rather common," "common," "very common," and "extremely common." For the sake of brevity, certain liberties have been taken with the term "brown," especially in the keys, where it is often used as a general term for some shade of yellowish-brown or brown. In the same way, allowances must be made for the term "throughout" when used to indicate occurrence, which does not imply the actual presence of a given species on every mountain top or every desert within the region.

Our knowledge of the polypores of tropical North America has been obtained chiefly through the large number of exploring expeditions sent into this interesting region during the past fifteen years by the New York Botanical Garden.

W. A. MUrRILL.

New York Botanical Garden,

March I5, I9I5. 


\section{TROPICAL POLYPORES}

Including the pileate species occurring in Mexico, Central America, southern Florida, the Bermudas, the West Indies, and all other islands between North America and South America with the exception of Trinidad, Tobago, and Curaçao, whose flora is essentially South American.

\section{POLYPORACEAE}

Hymenophore annual or perennial; context fleshy-tough, corky, or woody; hymenium poroid or lamelloid, fleshy to woody, never gelatinous.

Hymenium porose.

Hymenophore annual. 1

Volva wanting.

Tribe r. Polyporeae.

Volva present.

Hymenophore perennial. ?

Hymenium furrowed.

Tribe 2. Volvatae.

Tribe 3. FomiteaE.

Tribe 4. Daedaleak.

Tribe r. Polyporear. Hymenophore variable in size and shape, fleshy-tough to corky, annual, sometimes reviving; surface encrusted or anoderm, glabrous or hairy, zonate or azonate; context fibrous, rarely punky, variously colored; tubes cylindric, sometimes splitting into teeth, usually thin-walled; spores rounded or oblong, brown or hyaline; cystidia frequently present; surface of pileus never conidia-bearing; stipe often present, variously attached.

Context white.

Hymenophore sessile.

Tubes hexagonal, arranged in radiating rows; context thin.

13. Hexagona.

Tubes alveolar; context thin, dry; surface zonate.

Tubes mostly shallow, marginal and obsolete; hy-

27. Favolus. menium hydnoid or irpiciform at a very early stage.

I. IRPICIPORUS.

1 Apparent or real exceptions occur at times in Earliella, Coriolopsis, and Hapalopilus.

2 Exceptions occur in Fomitella, Ganoderma, and Amauroderma. Porodaedalea is closely allied to the Daedaleae.

- Cerrena shows an irpiciform hymenium at maturity, much resembling species of Coriolus. Daedalea and Gloeophyllum sometimes show poroid forms that are very confusing. 
Tubes normally poroid, sometimes irpiciform from the rupture of the dissepiments at maturity.

Pileus very soft, spongy, and elastic throughout.

Hymenophore small; tubes large, not fragile.

Hymenophore of immense size; tubes small, fragile when dry.

Pileus more or less firm, flexible or rigid.

Context duplex, spongy above, firm below; surface sodden and bibulous.

4. SPONGIPORUS.

5. TOMOphagus.

7. SPONGIPELLIS.

Context not duplex as above.

Pileus fleshy-tough to woody and rigid.

Surface anoderm, rarely zonate.

Hymenium more or less smokecolored at maturity.

8. BJERKANDERA.

Hymenium white or pallid.

Context fleshy to fleshy-tough, friable when dry.

Context punky to corky, not friable when dry.

Surface pelliculose, often zonate.

Plants large, more than $5 \mathrm{~cm}$. in diameter; hymenium fleshcolored.

Plants small, $5 \mathrm{~cm}$. or less in diameter; hymenium white or pallid.

Pileus thin, leathery, and more or less

flexible; surface usually zonate.

Hymenophore normally pileate; tubes small and nearly always regular.

Hymenophore semiresupinate; tubes large and irregular.

Hymenophore stipitate.

Stipe compound.

Stipe simple.

Tubes large, hexagonal and radially elongate from the first.

Tubes not as above.

Pileus inverted, erumpent from lenticels.

Pileus erect or lateral, not erumpent.

Context duplex, spongy above, woody below.

6. TYROMYCES.

9. Trametes.

10. EARLIELLA.

II. RIGIDOPORUS.

2. Coriolus.

3. Coriolellus.

17. Grifol.A.

13. Hexagona.

I2. Porodisculus.

16. Abortiporus.

Context homogeneous, firm.

Surface zonate.

14. Microporellus.

Surface azonate.

15. POLYPORUS.

Context bright-colored, yellow or red.

Hymenophore sessile or subsessile.

Tubes red.

Tubes yellow.

Context thin; plants small.

I8. PYCNOPORUS.

19. Flaviporellus. 
Context thick; plants very large.

Hymenophore distinctly stipitate; context yellow.

Context brown.

Hymenophore sessile, rarely substipitate.

Spores hyaline.

Context light-brown.

Surface glabrous or nearly so.

Hymenium alveolate.

Hymenium normally poroid.

Surface distinctly hairy.

Tubes small and regular.

Tubes large and irregular.

Context dark-brown.

Context duplex, mostly of intricately woven black hairs.

Context simple.

Context friable.

Context tough.

Tubes yellow.

Tubes brown, rarely greenish.

Tubes entire.

Surface heavily bearded.

Surface not bearded.

Tubes soon splitting into teeth.

Tubes black.

Spores brown.

Hymenophore thin, dry, multizonate.

Hymenophore not as above.

Hymenophore stipitate.

Spores hyaline.

Spores brown.

Pileus inverted, pendant.

Pileus erect; stipe central.
20. LAETIPORUS.

21. Phafolopsis.
27. Favolus.

26. Hapalopilus.

23. CORIOLOPSIS.

24. Funalia.

25. TRIChaptUM.

33. Phaeolus.

28. Flaviporus.

29. Pogonomyces.

23. Coriolopsis.

22. Cerrenella.

30. NIGROPORUS.

31. Cycloporellus.

32. INONOTUS.

33. Phaeolus.

34. Coltriciella.

35. Coltricia.

Tribe 2. VolvataE. Hymenophore corky to woody, annual; surface smooth, encrusted; context corky; tubes cylindric, concealed at first by a volva, which is perforated at one or more points at maturity; spores hyaline.

Pileus sessile, subglobose, white or slightly reddish-brown. 36. CRYPTOPORUs.

Tribe 3. Fomiteas. Hymenophore large, woody, perennial, rarely small or annual; surface anoderm or encrusted, usually sulcate, sometimes varnished; context punky or woody, variously colored; tubes cylindric, usually thickwalled; spores rounded, smooth or verrucose, hyaline or brown; cystidia frequently present; surface of pileus conidia-bearing in a few species; stipe rarely present, the hymenophore usually being sufficiently elevated by its host. Annual forms and species in a few genera connect this group with the Polyporeae; while the tendency at times to produce a daedaleoid hymenium, shown especially in Porodaedalea, connects it with the Daedaleae.

Surface of hymenophore covered with reddish-brown varnish; context punky to corky.

46. Ganoderma. 
Surface of hymenophore not as above.

Hymenophore distinctly stipitate.

Hymenophore sessile or subsessile.

Context white, flesh-colored, or wood-colored.

45. Amauroderma.

Context olivaceous.

37. Fomes.

Context brown, latericeous, dark-purple, or black.

38. Fomitella.

Surface not encrusted; or, if so, context woody.

Context brown or latericeous.

Hymenium porose.

Spores hyaline.

Spores brown.

Hymenium porose-daedaleoid.

Context dark-purple or black.

Surface encrusted; context punky.

Spores hyaline or subhyaline.

Spores decidedly brown.

39. Pyropolyporus.

40. Fulvifomes.

4I. Porodaedalea.

42. Nigrofomes.

43. Elfvingiella.

44. Elfvingia.

Tribe 4. Daedaleae. Hymenium annual, very rarely perennial, coriaceous to woody, variable in size; surface anoderm, hairy or glabrous, variously marked; context white or brown, fibrous, woody, or punky; hymenium exceedingly variable, normally labyrinthiform or lamelloid, but often poroid or even irpiciform, never stratified; spores smooth, brown or hyaline. Poroid and irpiciform plants of this group are difficult to separate from certain species of Polyporeae, forms of Daedalea confragosa in particular being troublesome to the beginner. On the other hand, there is little to cause confusion between this group and the Fomiteae, if we except the single distinctly perennial species of Daedalea and the daedaleoid forms of Porodaedalea.

Context white or wood-colored.

Hymenium labyrinthiform, often becoming lamellate or irpiciform.

Hymenium very soon becoming irpiciform.

Hymenium rarely becoming irpiciform and then not until maturity.

Hymenium lamellate from the first, not becoming irpiciform.

Context brown.

47. Cerrena.

48. Daedalea.

49. LeNzites.

50. Gloeophyllum.

\section{IRPICIPORUS Murrill}

Hymenophore annual, epixylous, sessile, effused-reflexed, white or pallid throughout; surface anoderm, glabrous or velvety, not distinctly zonate, margin acute; context white, coriaceous or corky; hymenium hydnoid or irpiciform, with traces of shallow, obsolete tubes near the margin; spores smooth, hyaline.

Surface villose; margin not cirrhose.

I. I. lacteus.

Surface glabrous; margin cirrhose.

2. I. cubensis. 


\section{IRPICIPORUS LACTEUS (Fries) Murrill}

Pileus extensively effused, shortly reflexed, imbricate, dimidiate, laterally connate, $0-1.5 \times I-4 \times 0 . I-0.2 \mathrm{~cm}$.; surface white, subzonate, concentrically furrowed in large specimens, villose; margin very thin, deflexed, undulate to lobed; context membranous, less than I mm. thick; tubes short, irregular, white to isabelline, $\mathrm{I}-3 \mathrm{~mm}$. long, mouths angular, about 2 to a mm., edges uneven, soon splitting into teeth, which are compressed, pointed, fimbriate, dentate to incised; spores cylindric, slightly curved, smooth, 6-7 $\times 2-3 \mu$.

Frequent on dead wood, especially in the mountains, in Cuba, Jamaica, Porto Rico, and eastern Mexico.

\section{IRPICIPORUS CUBENSIS (Berk. \& Curt.) Murrill}

Pileus reniform, sessile, $0.5-0.7 \times 1.5 \times 0.2 \mathrm{~cm}$.; surface glabrous, radiate-lineate, white to isabelline, azonate, smooth; margin deflexed, cirrhose, concolorous; context white, fragile, less than I mm. thick; tubes I-1.5 mm. long, white to discolored, 3-4 to a mm., very soon splitting into teeth, which are compressed, pointed and dentate.

Found once on dead wood in Cuba and once on a dead tree trunk at Cinchona, Jamaica. Specimens from Guadeloupe referred to this species by Duss are considerably larger, measuring $2 \mathrm{~cm}$. long and $3 \mathrm{~cm}$. broad.

\section{CORIOLUS Quél.}

Hymenophore annual, epixylous, sessile, usually zonate, anoderm, hairy or glabrous; context thin, white, flexible, fibrous, leathery; tubes thin-walled, white, at length splitting into irpiciform teeth in several species, mouths polygonal or irregular; spores smooth, hyaline.

Tubes more or less entire, at least until the hymenophore is quite old.

Surface of pileus wholly or partly glabrous when mature or clothed only with inconspicuous hairs.

Pileus not entirely glabrous at maturity.

Pileus marked at maturity with glabrous zones of a different color from the rest of the surface.

Glabrous zones large, numerous, conspicuously and variously colored.

I. C. versicolor.

Glabrous zones small and comparatively inconspicuous. 


\section{Tropical Polypores}

Hymenium white or yellowish.

Hymenium fuscous.

Pileus not marked with glabrous zones, but nearly uniform in color and rarely shining.

Hymenophore semiresupinate, narrowly reflexed.

Reflexed portion of pileus o- $5 \mathrm{~mm}$.'broad. Reflexed portion of pileus $\mathrm{I}-2 \mathrm{~cm}$. broad.

Hymenophore normally pileate, sometimes decurrent.

Surface entirely devoid of zones or concentric furrows.

Pileus 2-3 cm. broad.

Pileus 7-Io cm. broad.

Surface somewhat zonate or concentrically furrowed.

Tubes I mm. or less long.

Tubes 3-4 $\mathrm{mm}$. long.

2. C. subpavonius.

3. C. sector.

4. C. depauperatus.

5. C. tepeitensis.

6. C. pallidofulvellus.

7. C. xuchilensis.

8. C. delectans.

9. C. concavus.

Pileus entirely glabrous at maturity.

Surface marked with numerous brown and tawny zones.

Surface white to isabelline or pale-avellaneous, sometimes with darker spots or lines.

Hymenium lilac-colored, often faded in herbarium specimens.

Hymenium white or yellowish, sometimes discolored.

Margin of pileus entire or lobed, not becoming fimbriate or lacerate at maturity.

Pileus extremely thin, less than I mm., very flexible.

12. C. haedinus.

Pileus not as above.

Surface and tubes milk-white throughout, even in dried specimens.

10. C. armenicolor.

II. C. brachypus.

Surface and tubes not as above.

Surface white to pale-cinereous; tubes 7-8 to a $\mathrm{mm}$.

14. C. hondurensis.

Surface stramineous to ochroleucous.

Pileus I-2 mm. thick; tubes less than $\mathrm{I} \mathrm{mm}$. long.

Pileus 2-5 mm. thick; tubes more than $\mathrm{I} \mathrm{mm}$. long.

13. C. substipitatus.

15. C. pertenuis.

I6. C. ochrotinctellus. 


\section{Tropical Polypores}

Surface ochroleucous or ochraceous with pale-avellaneous zones.

17. C. Hollickii.

Surface isabelline-fulvous; tubes becoming fulvous.

Margin of pileus very thin, becoming fimbriate or lacerate at maturity.

18. C. leiodermus.

19. C. membranaceus.

Surface of pileus clothed entirely with a conspicuous hairy covering.

Pileus $5 \mathrm{~mm}$. or more thick; surface roughly hirsute.

20. C. nigromarginatus.

Pileus much thinner.

Hymenium becoming wholly or partly fuscous; tubes broad and very shallow.

Surface latericeous-fulvous, except near the margin.

Surface white to cinereous or isabelline.

Tubes regular in shape and size.

Tubes irregular both in size and shape.

Hymenium not becoming fuscous.

Surface more or less purple, marked with a few concentric lines.

21. C. fulvo-umbrinus.

22. C. pinsitus.

23. C. sericeohirsutus.

24. C. scutatus.

Surface white to yellowish or grayish.

Pileus effused-reflexed, projecting 3-7 $\mathrm{mm}$.

25. C. effusus.

Pileus dimidiate, projecting $2-5 \mathrm{~cm}$.

Tubes small, $4-6$ to a $\mathrm{mm}$.

Tubes large, $\mathrm{I}-2$ to a $\mathrm{mm}$.

26. C. pavonius.

27. C. orizabensis.

Tubes soon breaking up into long, irpiciform teeth.

Plants large, 10-20 cm. wide and 5-10 $\mathrm{mm}$. thick.

Plants much smaller and always very thin.

Pileus I cm. or less broad.

Pileus flabelliform, glabrous.

Pileus subcampanulate, pendulous, erumpent, finely tomentose.

28. C. maximus.

29. C. sobrius.

3o. C. cyphelloides.

Pileus more than I $\mathrm{cm}$. broad.

Surface white, minutely fibrose-lacerate.

Surface ashy-white, villose.

31. C. irpiciformis.

Surface wood-colored, tomentose.

32. C. abietinus.

33. C. prolificans.

\section{Coriolus versicolor (L.) Quél.}

Pileus densely imbricate, very thin, dimidiate, conchate, 2-4 $\times 3-7 \times 0.1-0.2 \mathrm{~cm}$.; surface smooth, velvety, shining, marked with conspicuous, glabrous zones of various colors, mostly latericeous, bay, or black; margin thin, sterile, entire; context thin, membranous; tubes punctiform, less than I $\mathrm{mm}$. long, white to isabelline within, mouths circular to angular, regular, even, 4-5 to a mm., edges thick and entire, becoming 
thin and dentate, white, glistening, at length opaque-isabelline or slightly umbrinous; spores allantoid, $4-6 \times 1-2 \mu$.

Occasional to frequent on dead wood in the mountains of Mexico and the West Indies.

\section{Coriolus subpavonius Murrill}

Pileus rather thin, coriaceous, slightly flexible when young, becoming rigid, especially on reviving the second season, dimidiate or flabelliform, subimbricate, applanate or slightly conchate, 2-3 $32-3 \times 0.1-0.3 \mathrm{~cm}$.; surface broadly zonate, finely tomentose to subglabrous in zones, pallid or cremeous, becoming bay or olive with age in zones or behind, margin thin, undulate or lobed, pallid; context white, fibrous, firm, I mm. thick; tubes punctiform, very short at first, later becoming I $\mathrm{mm}$. or more long, white to stramineous within and without, mouths glistening, very regular, circular, minute, 8-1o to a mm., edges rather thick, firm, entire.

Collected once on dead wood in a moist virgin forest near Jalapa, Mexico.

3. Coriolus sector (Ehrenb.) Pat.

Pileus rather thin, flexible, flabelliform, often spuriously short-stipitate, imbricate, laterally connate, $2-4 \times 3^{-6} \times 0.1-$ $0.2 \mathrm{~cm}$.; surface multizonate, finely radiate-striate, of ten plicate, silky, usually shining, more or less tomentose near the base, avellaneous to isabelline; margin entire to fimbriate or deeply dissected, sometimes palmate; context thin, coriaceous, avellaneous, about $0.5 \mathrm{~mm}$. thick; tubes $0.5-1 \mathrm{~mm}$. long, avellaneous within, mouths angular, irregular, very variable in size, 2-6 to a $\mathrm{mm}$., edges thin, dentate to lanceolate, avellaneous to fuliginous; spores globose, $3 \cdot 5-5 \mu$.

Common on dead wood throughout, and easily known by its avellaneous to fuliginous surface and tubes.

\section{Coriolus depauperatus (Pat.) Murrill}

Pileus widely effused, narrowly reflexed, very thin, soft, spongy, flexible, the reflexed portion $0-5 \mathrm{~mm}$. broad, I $\mathrm{mm}$. or less thick; surface white, spongy-tomentose, azonate; margin very thin, concolorous, broadly sterile at times; context white, membranous; tubes punctiform, very short, white within, mouths circular to slightly angular, 5 to a mm., edges thin, entire to dentate, white, becoming very pale yellowish on drying. 
Found a few times on dead trunks in Cuba, as well as in Venezuela.

\section{Coriolus tepeitensis Murrill}

Pileus mostly resupinate, subcircular to irregular, the margin detached from the substratum and slightly elevated at times, the reflexed portion, which is usually present, narrow, conchate, thin, flexible, reaching $6 \mathrm{~mm}$. in length and $\mathrm{I}-2 \mathrm{~cm}$. in width; surface irregularly and narrowly sulcate-zonate, subglabrous, uneven, varying from pallid to ochraceous or pale-avellaneous; context thin, whitish, punky; tubes white within, I-2 mm. long, mouths very regular, 5-6 to a mm., stramineous, subcircular, corky, glistening, edges entire, at first thick, becoming thinner with age.

Collected three times on dead fallen branches in the Tepeite Valley, near Cuernavaca, Mexico.

\section{Coriolus pallidofulvellus Murrill}

Pileus imbricate, decurrent, dimidiate, conchate, thin, flexible, I-I. $5 \times 2-3 \times 0 . I-0.2 \mathrm{~cm}$.; surface isabelline to pale-fulvous, slightly virgate with striate fibers, finely tomentose, azonate; margin thin, subentire, incurved when dry; context punkyfibrous, tough, pallid, less than I mm. thick; tubes very short, less than I mm., mouths angular, irregular, flaccid, 2-4 to a $\mathrm{mm}$., edges thin, dentate to lacerate, white to fulvous.

Occasional on dead wood in Cuba.

\section{Coriolus xuchilensis Murrill}

Pileus semiresupinate, imbricate, inconspicuous, the reflexed portion conchate, rather thin, 3-5 $\times$ 7-I0 $\times 0.1-0.2 \mathrm{~mm}$.; surface azonate, silky or pubescent, very pale fulvous fading to pallid on drying, margin undulate, acute, inflexed and slightly discolored on drying; context soft-fibrous, thick behind, very thin in front, pallid; tubes about I mm. long, dull-whitish within and without, mouths angular, somewhat irregular, $3-5$ to a mm., edges thin, entire, glistening.

Collected once on a dead trunk in a coffee plantation at Xuchiles, near Cordoba, Mexico.

\section{Coriolus delectans Murrill}

Pileus confluent-effused, subimbricate, broadly reflexed and expanded, dimidiate to reniform, applanate or convex above, concave below, very thin, soft, tough, very flexible, $2-4 \times 3^{-}$ 
$6 \times 0.1 \mathrm{~cm}$.; surface villose-tomentose, uniformly milk-white, marked with a few, indistinct, concentric furrows of variable width and depth; margin very thin, flaccid, entire to undulate, rarely lobed; context white, soft, tough, less than I mm. thick; tubes punctiform, white, less than I $\mathrm{mm}$. long, mouths angular, irregular, 4-5 to a $\mathrm{mm}$., soon lacerate, but not becoming irpiciform, edges very thin, flaccid, lacerate-dentate, white, stramineous in dried specimens.

Collected once on a dead hardwood trunk in the forest near Alto Cedro, Cuba.

\section{Coriolus concavus Murrill}

Pileus deeply concave, scutate-conchate, narrowly or rather broadly attached behind or by the vertex, rather thick, subrigid when dry but of corky texture, subimbricate, $2 \times 3-5 \times 0.2-0.8$ $\mathrm{cm}$.; surface finely tomentose, white, irregular, slightly once or twice sulcate, margin rather thick, entire or lobed, concolorous, not inflexed on drying; context punky, white; tubes rather long for the genus, reaching 3-4 $\mathrm{mm}$., at first white, changing to cremeous, mouths irregular, angular, large, 2 to a mm., edges thin, uneven to slightly dentate.

Collected once on dead wood in a moist forest near Orizaba, Mexico.

io. Coriolus armenicolor (Berk. \& Curt.) Pat.

Pileus very thin, flexible, flabelliform, attenuate behind, 2-3 $\times$ I.5-3.5 $\times 0.1 \mathrm{~cm}$., surface multizonate, minutely longitudinally striate, shining, glabrous, radiately plicate, at least when dry, isabelline with fulvous or pale-bay zones; margin very thin, lobed or incised; context membranous, coarsely fibrous, white, less than I mm. thick; tubes less than $0.5 \mathrm{~mm}$. long, white to pallid within, mouths angular, minute, $7-8$ to a mm., edges very thin, dentate, white when young, glistening, fulvous in dried specimens.

Occasional on dead wood in Cuba, Porto Rico, Jamaica, and Honduras.

\section{CoRiolus brachypus (Lév.) Murrill}

Pileus thin, coriaceous, firm, flexible, imbricate, dimidiate to flabelliform, sessile or attached by a short tubercle, $4^{-6} \times 5^{-12}$ $\times 0.2-0.4 \mathrm{~cm}$.; surface glabrous or nearly so, slightly radiately furrowed, multizonate, pallid to isabelline, usually rose-tinted; 
margin thin, narrowly sterile, undulate or slightly lobed; context punky-fibrous, pallid, I-3 mm. thick; tubes pale-roseous within, 2-3 $\mathrm{mm}$. long, very even and regular, mouths slightly angular, 5-6 to a mm., edges thin, slightly dentate, tough, firm, white or rose-tinted when young, becoming avellaneous-roseous at maturity, fading in herbarium specimens.

Common throughout on dead wood, and readily recognized by its beautiful rosy tints.

\section{i2. Coriolus haedinus (Berk.) Pat.}

Pileus very thin and very flexible, almost as thin as paper at times, dimidiate to circular, according to its relation to the substratum, applanate or conchate, laterally connate, decurrent, I-2 $\times 2-3 \times 0.02-0.05 \mathrm{~cm}$.; surface white to slightly yellowish, densely concentrically furrowed, slightly spongy between the furrows, glabrous; margin thin, undulate to lobed, fertile; context white, membranous; tubes short, less than I mm. long, mouths angular, nearly regular, 4-5 to a mm., with a tendency to appear much elongated radially by the partial separation of the transverse walls, edges very thin, fimbriate-dentate.

Occasional on dead wood in Cuba and Porto Rico, as well as in Brazil.

\section{I3. CoRiolus substipitatus Murrill}

Pileus flabelliform, attached by a short tubercle, applanate, rather thin, flexible, of very light weight, $4 \times 5-7 \times 0.2-0.3 \mathrm{~cm}$.; surface milk-white, opaque, glabrous, somewhat uneven, especially behind, faintly zonate in front; margin acute, concolorous, entire to slightly lobed; context thin, soft, punky, milk-white, I mm. thick; tubes I-I.5 mm. long, white within, firm and elastic, glistening, mouths circular to angular, very regular, 6-7 to a $\mathrm{mm}$., edges thin, entire, white, becoming denticulate with age, slightly yellowish in dried specimens; spores globose, $3.5 \mu$.

Found once near Port Antonio, Jamaica, on a log in woods.

\section{Coriolus hondurensis Murrill}

Pileus very thin, slightly flexible, conchate-reniform, sessile, $7 \times 9 \times 0.2-0.3 \mathrm{~cm}$.; surface radiate-rugose, glabrous, subshining, white to pale-cinereous, with a few very narrow, slightly darker, concentric lines; margin thin, white, lobed, inflexed on drying; context very thin, white, fibrous, less than I mm. thick; tubes 2-3 $\mathrm{mm}$. long, slender, white to pale-isabelline within, mouths minute, angular, regular, glistening, $7-8$ to a $\mathrm{mm}$., 
edges thin, dentate, white to dark-isabelline, fulvous in dried specimens.

Found once on dead wood near Punta Gorda, British Honduras.

\section{Coriolus PERTEnUis Murrill}

Pileus very thin, coriaceous, flexible, dimidiate, nearly plane, sessile by a narrow base, $2.5 \times 3 \times 0 . I-0.2 \mathrm{~cm}$.; surface slightly concentrically furrowed, glabrous, stramineous; margin thin, entire, sterile, cremeous; context white, fibrous, membranous; hymenium ochraceous when viewed at an angle, nearly fulvous when looked at perpendicularly; tubes punctiform, less than I $\mathrm{mm}$. long, mouths very regular, angular, about 5 to a mm., edges thin, entire.

Collected once at Chester Vale, Jamaica, on dead wood.

\section{I6. CoRIOLUS OCHROTINCTELlus Murrill}

Pileus subimbricate, dimidiate or flabelliform, applanate or conchate, sessile to substipitate, rigid, more or less brittle, 2-6 $\times 3-9 \times 0.2-0.5 \mathrm{~cm}$.; surface glabrous, smooth, subshining, ochroleucous with ochraceous zones; margin papery-thin, fragile, of ten incurved, entire or undulate, rarely lobed; context milkwhite, fleshy-tough, somewhat friable, I-3 mm. thick; tubes I-3 mm. long, slender, white to ochroleucous within, mouths very regular, angular, $4-5$ to a $\mathrm{mm}$., edges thin, entire, white to ochraceous or isabelline; spores globose, $3 \mu$.

Occasional on dead wood in southern Florida, Cuba, and Porto Rico.

\section{CoRIOlus Hollickir Murrill}

Pileus imbricate, substipitate, attached by a short, scutellate disk or tubercle, flabelliform, flexible to nearly rigid, rather thick for the genus, $4-6 \times 5^{-7} \times 0.5 \mathrm{~cm}$.; surface slightly radiaterugose, multizonate, shallowly concentrically sulcate, opaque, glabrous, ochroleucous or ochraceous to pallid with pale-avellaneous zones; margin subacute, entire, fertile, concolorous; context milk-white, soft-corky, homogeneous, about $2 \mathrm{~mm}$. thick; tubes stramineous within and without, becoming isabelline on drying, 2-3 mm. long, corky, rigid, mouths regular, nearly circular, glistening, 4-5 to a mm., edges thin, entire.

Collected once at Union Hill, near Moneague, Jamaica, on a $\log$ in the woods; once in Porto Rico; and four times at Jalapa, Mexico. 


\section{I8. Coriolus leiodermus (Mont.) Murrill}

Pileus thin, tough, leathery, flexible, dimidiate, of ten decurrent, conchate, $3-4 \times 4-6 \times 0.1 \div 0.3 \mathrm{~cm}$.; surface multizonate, slightly silky, becoming glabrous, minutely furrowed radiately, isabellinefulvous; margin thin, entire, pallid; context white to pallid, punky-fibrous, $0.5-1.5 \mathrm{~mm}$. thick; tubes $\mathbf{I}-\mathbf{I} .5 \mathrm{~mm}$. long, pallid within, mouths circular to angular, very regular, variable in size, $3^{-6}$ to a mm., edges thin, firm, entire to dentate, pallid to umbrinous-fulvous, glistening; spores globose, 3-4 $\mu$.

Occasional on dead wood in Nicaragua and Guiana.

\section{Coriolus membranaceus (Sw.) Pat.}

Pileus very thin, densely imbricate, dimidiate or flabelliform, conchate, $2-4 \times 3-6 \times 0.1-0.2 \mathrm{~cm}$.; surface multizonate, finely radiate-furrowed, short-tomentose to glabrous and subshining, white or pallid with slightly darker zones; margin very thin, undulate or lobed, usually splitting with age; context white, fibrous, $0.5^{-1.5} \mathrm{~mm}$. thick; tubes very short, less than I mm., white to discolored within, mouths angular, 4-6 to a mm., edges very thin, denticulate, fimbriate with age, white to isabelline or nearly fulvous in dried specimens; spores globose, 4-5 $\mu$.

Extremely common throughout on various forms of dead wood.

\section{Coriolus nigromarginatus (Schw.) Murrill}

Pileus confluent-effused, more or less imbricate, dimidiate, applanate, corky-leathery, rather thick, flexible or rigid, 3-5 $\times 5^{-8} \times 0.3-0.8 \mathrm{~cm}$.; surface conspicuously hirsute, isabelline to cinereous, concentrically furrowed and zoned; margin at length thin, often fuliginous, sterile, finely strigose-tomentose, entire or undulate; context white, thin, fibrous, spongy above, I-4 mm. thick; tubes white, I-2 mm. long, mouths circular to angular, 4 to a mm., quite regular, edges thin, firm, tough, entire, white to yellowish or umbrinous; spores cylindric, slightly curved, $2.5-3 \mu$.

Common throughout on various forms of dead wood.

\section{I. CoRIolus fUlvo-Umbrinus Murrill}

Pileus laterally connate, semicampanulate, umbonate-attached, thin, slightly flexible, $2 \times 2-3 \times 0.1 \mathrm{~cm}$., broader by confluence; surface strigose-tomentose, multizonate, somewhat radiaterugose, uneven, latericeous-fulvous, the zones very slightly darker; margin and new growth white to avellaneous, hirsute, zonate, slightly lobed, sometimes proliferous; context thin, mem- 
branous, pallid; tubes punctiform, white within, mouths large, hexagonal, irregular, $2-3$ to a mm., edges entire to laceratedentate, the divisions distinctly spinose, white to umbrinous.

Found once on old timber near the beach at Havana, Cuba.

\section{Coriolus pinsitus (Fries) Pat.}

Pileus thin, flexible, confluent-effused, dimidiate or flabelliform, of ten umbonate-affixed, $2-4 \times 3-7 \times 0.1 \mathrm{~cm}$.; surface isabelline to pale-cinereous, glistening, multizonate, velvety-hirsute; margin thin, undulate to lobed, sterile, inflexed on drying; context white, membranous; tubes short, white to discolored within, less than I $\mathrm{mm}$. long, mouths hexagonal, very regular, $2-3$ to a $\mathrm{mm}$., edges thin, denticulate, white to discolored, of ten becoming fuliginous; spores oblong, $6-7 \times 3 \mu$.

Extremely common on dead wood throughout. The variation in the color of the tubes may be very confusing unless one happens to find white and blackish tubes on the same hymenophore. It was first described from Jamaica as Boletus villosus Sw.; not $B$. villosus Huds.

\section{Coriolus SERICEOHIRSUTUS (Klotzsch) Murrill}

Pileus very thin, flexible, effused-confluent, sometimes wholly resupinate, conchate-reflexed, $0-3 \times 2-6 \times 0.05-0.1 \mathrm{~cm}$.; surface conspicuously silky-villose to strigose-hirsute, multizonate, palebrown to hoary, with slightly darker zones; margin thin, entire or undulate, dentate or eroded with age; context thin, white, membranous; tubes shallow, I-I.5 $\mathrm{mm}$. deep, white to slightly discolored within, mouths hexagonal, irregular, very variable in size, $0.3^{-1} \mathrm{~mm}$. in diameter, edges thin, denticulate to dentate, white to discolored, sometimes becoming umbrinous; spores $6 \times 4 \mu$.

Common on dead trunks and branches of red cedar in southern Florida and the Bermudas, and occasional in the Bahamas and Cuba.

\section{Coriolus scutatus Murrill}

Pileus scutate-conchate, fixed at the center, thin, slightly flexible, coriaceous, $5-7 \mathrm{~cm}$. broad, $\mathrm{I}-2 \mathrm{~mm}$. thick; surface villosetomentose, isabelline to dark-purple or black, marked with a few narrow, concentric lines; margin thin, sterile, pallid, entire or undulate, inflexed when dry; context thin, soft-fibrous, less than I $\mathrm{mm}$. thick; tubes short, white, less than I mm. long, mouths 
circular, 5 to a mm., edges rather thick, firm, entire, white to discolored, purplish-spotted when bruised; spores ovoid, $4 \times 3 \mu$.

Found once on dead wood in Mexico.

\section{Coriolus effusus Murrill}

Pileus very thin, flexible, broadly confluent, effused, shortly reflexed, the reflexed portion convex, laterally elongate, projecting 3-7 mm.; surface finely tomentose, white or pale-yellowish, slightly sulcate, especially near the margin, which is broadly sterile, milk-white, undulate; context thin, white, membranous; hymenium very irregular, varying according to the position of the tubes, white or slightly yellowish; tubes very short, mouths circular or much elongated radially, edges thin, becoming lacerate-dentate or somewhat irpiciform.

Frequent on dead wood in Jamaica.

\section{Coriolus pavonius (Hook.) Murrill}

Pileus thin, coriaceous, flexible, dimidiate, imbricate, conchate, 3-5 $\times$ 4-7 $\times 0.1-0.2 \mathrm{~cm}$.; surface multizonate, strigose-velvety, pallid, becoming pale-cinereous with age; margin thin, white or yellowish, sterile, entire or undulate; context white, fibrous, about I mm. thick; tubes punctiform, less than I mm. long, white to slightly yellowish within, mouths very regular in normally developed specimens, circular to slightly angular, $4^{-6}$ to a mm., edges white to ochraceous, very thin, denticulate; spores globose, $4 \mu$.

Common throughout on dead hardwood logs and sticks.

\section{Coriolus ORIZABENSIs Murrill}

Pileus many times imbricate, very slightly decurrent at times, sessile, dimidiate or fan-shaped, conchate, thin, flexible, $2-3 \times$ $3-5 \times 0.2-0.3 \mathrm{~cm}$.; surface white to slightly yellowish-discolored, uneven, rugose, two or three times slightly sulcate, obscurely zonate, appressed-fibrillose, margin undulate, somewhat abruptly thickened, finely tomentose, conspicuously appressed-fibrillose, subconcolorous; context white, fibrous, I mm. thick; tubes 2 $\mathrm{mm}$. long, pallid within, mouths large, irregular, glistening, white to discolored, 2 to a $\mathrm{mm}$. near the margin, I to a $\mathrm{mm}$. behind, edges thin, lacerate-dentate.

Collected once on dead wood in moist woods near Orizaba, Mexico. 


\section{Coriolus maximus (Mont.) Murrill}

Pileus very large, flexible or rigid, leathery, dimidiate, imbricate, $5^{-12} \times 10-20 \times 0.2-0.5 \mathrm{~cm}$.; surface conspicuously villose-tomentose to partially glabrous, concentrically furrowed, white or cremeous, becoming hoary; margin thin, entire to lobed; context soft and spongy above, tough and fibrous below, white, $\mathrm{I}-3 \mathrm{~mm}$. thick; tubes $\mathrm{I}-2 \mathrm{~mm}$. long, white to cremeous within, soon becoming irpiciform, mouths circular when very young, 3-4 to a mm., edges white to ochraceous, firm, fimbriatedentate, soon splitting into sharp teeth.

Very common and conspicuous on dead trunks throughout the region.

\section{Coriolus sobrius (Berk. \& Curt.) Murrill}

Pileus very thin, small, flabelliform, imbricate, $\mathrm{I} .5 \times \mathrm{I} \times$ 0.I cm.; surface very smooth, glabrous, subzonate, isabelline to pale-chestnut, variegated, margin very thin, entire; context membranous, white, less than $0.5 \mathrm{~mm}$. thick; tubes nearly I $\mathrm{mm}$. in length, shorter near the margin, soon becoming irpiciform, mouths rather large for the size of the pileus, irregular, radially elongate, angular, 2-4 to a mm., edges white to discolored, entire to lacerate.

Found once on dead wood in Cuba.

\section{CoRIOLUS CYPHELlOIDES (Fries) Murrill}

Pileus minute, erumpent through the cortex, thin, coriaceous, pendulous, subcampanulate to unguliform, attenuate behind, $0.6 \times$ I $\times 0.2-0.3 \mathrm{~cm}$.; surface finely tomentose to nearly glabrous, silky-shining, finely zonate near the margin, which is thin, fertile, concolorous; context white, fibrous, less than 0.5 $\mathrm{mm}$. thick; tubes rather long, $1.5-2 \mathrm{~mm}$., white within, large and irregular, soon becoming irpiciform, mouths 2-3 to a mm., edges white or pallid, thin, dentate to lacerate.

Found once on dead wood in Mexico.

\section{I. CORIOLUS IRPICIFORMIS Murrill}

Pileus white throughout or slightly greenish behind, probably owing to algae, gregarious, elongate-spatulate, narrowly or broadly attached, $4-7 \times 2.5-4 \times 0.2 \mathrm{~cm}$.; surface slightly zonate, minutely fibrose-lacerate, margin lacerate, fertile; context very thin, fibrous; tubes about $2 \mathrm{~mm}$. long, mouths large, angular, soon becoming irpiciform, edges acute, separating into irregular, toothed plates. 
Collected once on dead chips in moist woods near Orizaba, Mexico.

\section{Coriolus abietinus (Dicks.) Quél.}

Pileus effused-reflexed, the reflexed portion thin, tough, flexible to nearly rigid, $0.5-1.5 \times 1-3 \times 0.05-0.1 \mathrm{~cm}$.; surface obsoletely zonate, grayish-white, villose; margin thin, undulate to lobed, fimbriate with age, incurved on drying; context very thin, white, membranous; tubes uneven, irregular, soon becoming irpiciform, mouths variable in size, $2-3$ to a $\mathrm{mm}$., edges thin, laceratedentate, unequal, pallid or violet, fading with age, somewhat flesh-tinted in dried specimens; spores globose, 4.5-5.5 $\mu$.

Frequent on dead pine trunks in southern Florida and in the mountains of Cuba and Mexico.

\section{CoRIOLUS PROLIFICANS (Fries) Murrill}

Pileus exceedingly variable, sessile or affixed by a short tubercle, dimidiate to flabelliform, broadly or narrowly attached, 2-5 $\times 2-6 \times 0.1-0.3 \mathrm{~cm}$.; surface finely villose-tomentose, smooth, white or slightly yellowish, marked with a few narrow, indistinct, latericeous or bay zones; margin thin, sterile, entire to lobed; context very thin, white, fibrous; tubes $\mathrm{I}-3 \mathrm{~mm}$. long, white to discolored within, mouths angular, somewhat irregular, 3-4 to a mm., usually becoming irpiciform at an early stage, edges acute, dentate, becoming lacerate, white to yellowish or umbrinous.

Frequent to common on dead wood throughout most of the region, sometimes assuming peculiar branched forms.

\section{CORIOLELLUS Murrill}

Hymenophore small, dry, annual, epixylous, semiresupinate; surface anoderm, usually azonate; context white, thin, fibrous to corky; hymenium concolorous; tubes thin-walled, usually rather large and irregular, dentate, but not irpiciform; spores smooth, hyaline.

\section{Coriolellus Sepium (Berk.) Murrill}

Pileus small, dimidiate, laterally connate, narrowly attached when young, becoming decurrent and of ten effused, $0.5-1 \times 1-3.5$ $\times 0.2-0.5 \mathrm{~cm}$.; surface white or pale-wood-colored, finely tomentose to glabrous, subzonate, smooth or broadly radiately furrowed; margin thin or tumid, entire to undulate; context white, I-2 mm. thick, soft-corky; tubes white, $2-3 \mathrm{~mm}$. long, mouths angular, uneven, irregular, sometimes slightly sinuous, $\mathbf{I}-2$ to a 
mm., edges thin, undulate to dentate, white; spores oblong, $12 \times 5 \mu$.

Frequent on structural timber and other dead wood in the mountains of Cuba, Jamaica, Montserrat, Mexico, and Costa Rica. An isabelline, rather irregular form, varying to entirely resupinate, is often difficult to associate with the typical form of this species.

\section{SPONGIPORUS Murrill}

Hymenophore small, annual, epixylous, sessile, dimidiate, pulvinate; surface white, anoderm to subpelliculose, azonate, soft and elastic; context white, extremely soft and spongy throughout; hymenium rigid, somewhat discolored; tubes large, irregular, thin-walled, lacerate; spores smooth, hyaline.

\section{Spongiporus altocedronensis Murrill}

Pileus very soft and spongy, broadly attached, decurrent, subimbricate, $0.5^{-1} \times \mathrm{I}^{-5}-3 \times 0.5^{-1} \mathrm{~cm}$.; surface white, azonate, smooth, villose; margin thick, broadly sterile; context white, very soft and spongy, radiate-fibrous, a few fibers being darker and firmer, 3-8 mm. thick; tubes shallow, ample, white to palereddish-brown, about $\mathrm{I} \mathrm{mm}$. in length, $\mathrm{I}-2$ to a mm., mouths quite irregular, angular, edges thin, uneven, dentate; spores globose, $4-4.5 \mu$.

Collected once on rotten wood at Alto Cedro, Cuba.

\section{TOMOPHAGUS Murrill}

Hymenophore very large, but of light weight, annual, epixylous, sessile, dimidiate, thick and pulvinate; surface pelliculose, glabrous, azonate, margin very obtuse; context very thick, soft and spongy throughout; tubes small, dark-colored, thin-walled, fragile; spores smooth, hyaline.

\section{Tomophagus Colossus (Fries) Murrill}

Pileus of immense size but of very light weight, dimidiate to reniform, sessile, convex above, 10-20 $\times 15-30 \times 7-10 \mathrm{~cm}$.; surface azonate, glabrous, smooth, pale-yellowish, at length covered with a very thin cuticle, which becomes cracked, wrinkled, and pallid with age; margin very obtuse, rounded, concolorous; context very soft and spongy, homogeneous, concentrically zonate, several centimeters thick; tubes minute, soft, white to discolored within, $2-5 \mathrm{~mm}$. long, mouths angular, 3-4 
to a mm., edges thin, dentate, white to discolored, umbrinous or fuliginous in herbarium specimens; spores obovoid, smooth, hyaline tinged with cream-olive, $15-18 \times 8-12 \mu$.

Found on stumps of Cedrela odorata once in Costa Rica and once in Yucatan.

\section{TYROMYCES P. Karst.}

Hymenophore annual, epixylous, sessile, anoderm, azonate, glabrous or nearly so; context white, fibrous, fleshy to fleshytough, rigid and friable when dry; tubes thin-walled, white or yellowish, mouths polygonal; spores smooth, hyaline.

Pileus large, $8 \mathrm{~cm}$. or more in diameter.

Tubes less than $5 \mathrm{~mm}$. long.

Pileus over $1 \mathrm{~cm}$. thick.

Pileus 3-5 mm. thick.

I. T. palustris.

2. T. Palmarum.

Tubes more than $5 \mathrm{~mm}$. long.

Surface of pileus very smooth.

Pileus white or slightly yellowish, unchanging; tubes small, entire.

Pileus becoming dark-sordid-bay throughout on drying; tubes 3 to a mm., lacerate.

Surface of pileus not very smooth; tubes minute; spores subglobose, $3 \mu$.

Pileus small, rarely exceeding $5 \mathrm{~cm}$. in diameter.

Pileus resinous or cartilaginous in appearance.

Pileus neither resinous nor cartilaginous in appearance.

Hymenium more or less bluish or bluish-spotted. Pileus effused-reflexed, white or pale-isabelline.

Pileus not effused-reflexed, more or less bluish.

Hymenium not tinged with blue.

Pileus white or nearly so, sometimes becoming slightly darker with age.

Surface entirely glabrous throughout. Pileus effused-reflexed, $1.5 \mathrm{~cm}$. broad. Pileus flabelliform, narrowly attached, $2.5 \mathrm{~cm}$. broad.

3. T. nivosellus.

4. T. Smallii.

5. T. graminicola.

6. T. versicutis.

7. T. semipileatus.

8. $T$. caesius.

9. T. semisupiniformis.

ro. $T$. cinchonensis.

Surface tomentose or pubescent, sometimes becoming nearly glabrous.

Tubes 4-5 to a mm.; species confined to high elevations.

Tubes 6-7 to a mm.; species confined to low elevations.

II. T. lacteus.

I2. T. leucomallus.

Pileus gilvous or some shade of fulvous.

Tubes 3-4 mm. long, much longer than the thickness of the very thin context.

13. T. albogilvus. 
Tubes short, about equal to the thickness of the context.

Hymenium subfulvous; spores ovoid, 3-4 $\mu$.

Hymenium white to pallid; spores cylindric, $4 \times I \mu$.

14. T. fulvitinctus.

15. T. duracinus.

\section{Tyromyces palustris (Berk. \& Curt.) Murrill}

Pileus dimidiate, convex above, plane or concave below, much thicker behind, subimbricate, fleshy-tough to rigid and somewhat friable when dry, $4-6 \times 8-10 \times \mathrm{I}-3 \mathrm{~cm}$.; surface smooth, glabrous, white to slightly yellowish, sometimes rough and tubercular behind; margin thin or thick, entire or undulate, white, becoming slightly discolored; context I-2 cm. thick, white, fleshy-fibrous when fresh, becoming firm and somewhat friable when dry; tubes $2-5 \mathrm{~mm}$. long, white to slightly yellowish within, about 4 to a mm., edges thin, white to yellowish, entire to dentate.

Occasional on pine trunks in southern Florida, the Bahamas, and Cuba.

\section{Tyromyces Palmarum Murrill}

Pileus very broad, thin, applanate, dimidiate, concave below, incurved on drying, fleshy-tough to rigid, 6-1o $\times$ 10-20 $\times 0.5^{-}$ $0.8 \mathrm{~cm}$.; surface glabrous, nearly smooth, slightly rugose or tubercular, white to cremeous; margin thin, undulate to lobed, fertile, concolorous; context white, fleshy-fibrous to rigid and somewhat fragile on drying, 3-5 mm. thick; tubes 2-4 $\mathrm{mm}$. long, white to yellowish within, mouths glistening, angular, regular, 3 to a mm., white to slightly yellowish and finally avellaneous, edges thin, entire; spores globose, $3 \mu$.

Found a few times on dead palm logs in Cuba and Jamaica.

\section{Tyromyces nivosellus Murrill}

Pileus simple, imbricate, dimidiate, semicampanulate to ungulate, very thick behind, convex above, concave below, 3-5 $\times$ 6-9 $\times 2-4 \mathrm{~cm}$.; surface smooth, glabrous, azonate, white or very slightly yellowish; margin thick, usually obtuse, entire or undulate, concolorous, darker when dry; context fleshy-tough, rigid, but slightly friable when dry, milk-white, $0.5^{-2.5} \mathrm{~cm}$. thick; tubes long and slender, about I cm., white to dull-yellowish within, mouths regular, angular, 3 to a mm., white to isabelline or umbrinous, edges firm, rather thin, entire; spores ellipsoid, $3.5 \times 6 \mu$.

Found a few times on palm trunks in Cuba and Porto Rico. 


\section{Tyromyces SmalliI Murrill}

Pileus compressed-ungulate, with a large umbo, broadly sessile, dimidiate, fleshy-tough, difficult to dry, 5-7 $\times 6-10$ $\times 2-5 \mathrm{~cm}$.; surface very smooth, glabrous, azonate, white to cremeous, changing to sordid-bay or blackish on drying; context fleshy-tough, zonate, watery, moist and flexible even in dried specimens, dull-white to sordid-avellaneous, tinged with flesh-color, $0.5^{-2.5} \mathrm{~cm}$. thick; tubes $5^{-8} \mathrm{~mm}$. long, 3 to a mm., rather large and irregular at times from the splitting of the dissepiments, partially collapsed, somewhat fragile, dark-bay throughout in dried specimens, edges thin, lacerate, fimbriate.

Found a few times on pine trunks in Florida.

\section{Tyromyces graminicola Murrill, sp. nov.}

Hymenophore forming a large oblong cluster about $30 \mathrm{~cm}$. long, $15 \mathrm{~cm}$. broad, and $10 \mathrm{~cm}$. high, very irregular and lobed by reason of its habitat, the lobes dimidiate, rather thin, uniting behind into a thick substipitiform base; surface fleshy, soft, uneven, glabrous, white, partly changing to yellowish or brownish on drying; context thin, white, becoming spongy-fragile and rose-tinted on drying; tubes fleshy, slender, angular, minute, white, becoming brownish and collapsing on drying, the edges very thin, becoming somewhat lacerate; spores subglobose, smooth, hyaline, about $3 \mu$.

Type collected in a clump of grass (Sporobolus?) in open loamy soil, near Harrington Sound, Bermuda, August 27September 21, 1912, Stewardson Brown \& N. L. Britton 834 (herb. N. Y. Bot. Gard.). The fungus was evidently living on the decaying grass roots, since no dead wood was present, either above or beneath the ground. No other specimens of this species have been collected.

\section{Tyromyces versicutis (Berk. \& Curt.) Murrill}

Pileus cespitose-imbricate, fleshy-tough, rigid when dry, dimidiate, decurrent behind, $0.5^{-1} .5 \times 3-4 \times 0.2-0.4 \mathrm{~cm}$.; surface rugose or tuberculose, finely tomentose, resinous-guttate, ochraceous to latericeous when dry; margin somewhat obtuse, sterile; context fleshy to rigid, pallid, $2 \mathrm{~mm}$. thick; tubes $\mathrm{I}-2 \mathrm{~mm}$. long, pallid to umbrinous, mouths circular to slightly angular, minute, 6 to a mm., edges thin, subentire, pallid to umbrinous.

Found a few times on dead wood in Cuba. 


\section{Tyromyces semipileatus (Peck) Murrill}

Pileus effused, largely resupinate, suborbicular or laterally elongate, very narrowly reflexed, the reflexed portion $0-1 \times 2-5$ $\times 0.3-0.5 \mathrm{~cm}$.; surface white or pale-isabelline, subvillose or scabrous, azonate; margin thin, undulate, sometimes inflexed; context white, fleshy-tough to fragile, 2-4 $\mathrm{mm}$. thick; tubes short, slender, white to yellowish within, mouths minute, circular to slightly angular, scarcely conspicuous, 7 to a mm., edges thin, very even, entire, white to pallid, often bluish-discolored in spots or blotches; spores subglobose, $6-8 \mu$.

Found on dead wood in the Tepeite Valley, Mexico, at an elevation of $2300 \mathrm{~m}$.

\section{Tyromyces caesius (Schrad.) Murrill}

Pileus dimidiate, imbricate, often narrowly attached, with a prominent umbo, variable in habit and size, soft, spongy when fresh, fragile when dry, $\mathrm{I}-2 \times 3-6 \times 0.5-1.5 \mathrm{~cm}$.; surface sodden, tomentose or villose-tomentose, azonate, murinous or griseous when fresh, becoming caesious or fading to nearly purewhite on drying, often nearly glabrous with age; context white, soft, friable, 5-8 mm. thick; tubes long and slender, 5-1o $\mathrm{mm}$. long, caesious within, collapsing, friable, mouths angular, 3-4 to a $\mathrm{mm}$., edges white or bluish-gray, very thin, dentate to long and sharply lacerate; spores elongate, $5-5.5 \times 1.5 \mu$.

Abundant on dead branches and trunks of Juniperus at Cinchona and New Haven Gap, Jamaica, and found also at Castleton, Jamaica, and in the Tepeite Valley, Mexico.

\section{Tyromyces SEMISUPINIFORMIS Murrill}

Pilei very abundant, imbricate, semiresupinate, the reflexed portion dimidiate or laterally extended, sometimes cuneate, convex above, slightly concave below, thin, very tough, I-I.5 cm. long, about $\mathrm{I} .5 \mathrm{~cm}$. broad, larger by confluence, $\mathrm{I}-3 \mathrm{~mm}$. thick; surface slightly sulcate-zonate, dull-ochroleucous behind, stramineous in front, glabrous, somewhat uneven, margin thin, sterile, undulate or lobed; context very thin, white, rigid when dry, only slightly friable; tubes ochroleucous, cremeous near the margin; mouths very minute, invisible to the unaided eye, circular to angular, edges entire, obtuse, becoming thin.

Collected once on the side of a large log in a moist virgin forest near Jalapa, Mexico. 


\section{IO. Tyromyces cinchonensis Murrill}

Pileus imbricate, flabelliform, attached by a narrow base, convex above, concave below, slightly flexible, becoming rigid and fragile when dry, $2.5 \times 2.5 \times 0.2-0.3 \mathrm{~cm}$.; surface milk-white, becoming suffused with avellaneous or very pale ardesiacous markings, perfectly glabrous, radiate-rugose; margin thin, undulate or eroded, deflexed on drying, concolorous; context white, thin, fibrous, rather tough for the genus; tubes white, much longer than the thickness of the context, averaging about $2 \mathrm{~mm}$., mouths angular, regular, white with an ashy tint, glistening, 5-6 to a mm., edges thin, becoming slightly lacerate-dentate; spores cylindric, curved at times, $4 \times 1.5^{-2.5} \mu$.

Collected twice at Cinchona, Jamaica, on dead coniferous wood.

\section{Tyromyces Lacteus (Fries) Murrill}

Pileus dimidiate, sessile, decurrent, convex, very soft, fleshy, becoming rigid when dry, $2-4 \times 5-8 \times 0.5^{-1.5} \mathrm{~cm}$.; surface milk-white, sometimes slightly discolored, azonate, finely tomentose or pubescent to nearly glabrous, more or less silky-striate; margin abruptly thin, inflexed, undulate, concolorous; context spongy-fibrous, very fragile when dry, 5-ro mm. thick, milkwhite, zonate at times; tubes quite long, slender, equaling the thickness of the context, 5-10 mm., milk-white within, mouths regular, angular, 4-5 to a mm., glistening, becoming lacerate and somewhat uneven, edges thin, dentate to sharply toothed, fragile, white to slightly yellowish; spores allantoid, $4-5 \times$ I-I.5 $\mu$.

Found on dead wood in the Tepeite Valley, near Cuernavaca, Mexico, at an elevation of $2300 \mathrm{~m}$.

\section{Tyromyces leucomallus (Berk. \& Curt.) Murrill}

Pileus soft, fleshy, very fragile when dry, dimidiate, sessile, convex, $3^{-5} \times 5^{-8} \times 0.5^{-1.5} \mathrm{~cm}$.; surface white to slightly cinereous, tomentose, appressed-fibrose when young; margin acute to slightly obtuse, sometimes inflexed, brown or black when bruised; context very soft and friable when dry, milkwhite, $0.5^{-1} \mathrm{~cm}$. thick; tubes white, 3-10 $\mathrm{mm}$. long, mouths minute, $6-7$ to a $\mathrm{mm}$., subcircular, edges white to pallid, subglistening, very thin and dentate to slightly lacerate.

Occasional on dead wood in Cuba, western Jamaica, and eastern Mexico. 
13. Tyromyces albogilvus (Berk. \& Curt.) Murrill

Pileus dimidiate to flabelliform, sometimes attached by a very narrow base, fleshy, rigid when dry, convex above, plane below, $2.5 \times 2.5-3 \times 0.4-0.7 \mathrm{~cm}$.; surface finely tomentose to resinousglabrous, gilvous when fresh, azonate, somewhat radiate-rugose; margin rather thick, of ten obtuse, entire, concolorous; context thin, white, fleshy to fragile, I mm. thick; tubes long, slender, 4-6 mm., white or pale-yellowish, mouths minute, angular, 6 to a mm., glistening, white or pale-yellowish, usually abruptly radially elongate near the margin, presenting the appearance of lamellae, edges thin, entire or slightly dentate.

Occasional on dead trunks in woods in Cuba, Porto Rico, Jamaica, and British Honduras.

\section{Tyromyces fulvitinctus (Berk. \& Curt.) Murrill}

Pileus fleshy-tough, rigid and fragile when dry, sessile, dimidiate, applanate, decurrent, $3-4 \mathrm{~cm}$. broad, 3-5 mm. thick; surface tomentose, alutaceous-fulvous, becoming glabrous behind, azonate, subsulcate, margin rather thick, fertile, entire or undulate; context homogeneous, corky-fragile, whitish to isabelline, I-2 $\mathrm{mm}$. thick; tubes short, $2-3 \mathrm{~mm}$. long, white to isabelline or avellaneous, mouths small, circular to angular, pale-umbrinous in dried specimens, edges thin, obtuse, entire; spores subglobose to ovoid, $3-4 \mu$.

Found a few times on dead wood in Cuba and once in Grenada.

\section{Tyromyces duracinus (Pat.) Murrill}

Pileus convex, rigid, hard, I.5-2.5 $\mathrm{cm}$. broad, 2-4 mm. thick; surface isabelline to pale-fulvous, darker near the margin, glabrous, marked with I-2 concentric furrows; margin inflexed, usually acute; context hard, fragile, white, I-2 mm. thick; tubes I $\mathrm{mm}$. long, white to discolored, mouths small, circular, 5 to a $\mathrm{mm}$., edges white to avellaneous, obtuse, entire; spores cylindric, $4 \times$ I $\mu$.

Found once in Guadeloupe on dead branches of Cecropia peltata.

\section{SPONGIPELLIS Pat.}

Hymenophore annual, epixylous, sessile, dimidiate, simple or imbricate, rather large; surface white, anoderm, sodden and bibulous; context white, duplex, spongy above, firm below; hymenium concolorous, tubes thin-walled; spores smooth, hyaline. 
Pileus I-2 cm. thick; tubes I-2 to a mm.

I. S. luridescens.

Pileus less than $\mathrm{I} \mathrm{cm}$. thick.

Tubes small, 6 to a $\mathrm{mm}$.

2. S. hydrophilus.

Tubes large, i-3 to a mm.

3. S. substuppeus.

\section{Spongipellis LuRIDESCENS Murrill}

Pileus compressed-ungulate, subimbricate, dimidiate, plane below, $3 \times 5 \times \mathrm{I}-2 \mathrm{~cm}$.; surface smooth, spongy-tomentose, azonate, isabelline to fulvous or fuliginous; margin rather thick, either acute or rounded, entire, sterile, concolorous; context soft, punky, white to discolored, 3-5 $\mathrm{mm}$. thick, 5-8 $\mathrm{mm}$. long, white to isabelline within, darker near the mouths, which are circular to angular, sinuous and slightly irpiciform behind, I-2 to a $\mathrm{mm}$., edges thin, firm, corky, entire to lacerate, isabelline to fuliginous with age; spores ellipsoid, 3-4 $\times$ I-2 $\mu$.

Found once at Hall's Delight, Jamaica, on old stumps.

\section{SpONGIPEllis hydrophilus (Berk. \& Curt.) Murrill}

Pileus thin, somewhat cespitose, flabelliform, attached by a tubercle, fleshy and very watery when fresh, becoming rigid and contorted when dry, 2-3 $\times 3-5 \times 0.2-0.4 \mathrm{~cm}$.; surface isabelline, finely hispid-tomentose, zonate and concentrically furrowed near the margin, which is very thin, incurved and bay to black in dried specimens; context fleshy to rigid and hard, white to slightly yellowish, I-2.5 mm. thick; tubes short, I-I.5 mm. long, white to isabelline within, mouths minute, circular to angular, 6 to a mm., collapsing, edges thin, dentate, white to isabelline, glistening.

Found a few times on logs in Cuba and British Honduras.

\section{Spongipellis substuppeus (Berk. \& Cooke) Murrill}

Pileus simple or imbricate, dimidiate, umbonate-sessile or broadly attached, decurrent, 3-4 $\times 4-7 \times 0.3-0.8 \mathrm{~cm}$.; surface floccose, substuppeous, spongy, azonate, pale-ochraceous to discolored; margin thin, entire, easily discolored; context soft, fibrous-spongy, white to discolored, $\mathrm{I}-3 \mathrm{~mm}$. thick; tubes $2-4$ $\mathrm{mm}$. long, white to pale-latericeous within, mouths large, irregular, angular, radially elongate at times, I-3 to a $\mathrm{mm}$., edges thin, dentate, white to umbrinous; spores globose, 5-7 $\mu$.

Found a few times on dead wood in Cuba, as well as in Brazil.

\section{BJERKANDERA P. Karst.}

Hymenophore annual, epixylous, sessile, anoderm, glabrous, azonate, corky; context white, tough or woody, not friable when 
dry; tubes thin-walled, more or less smoke-colored, mouths polygonal; spores smooth, hyaline.

Hymenium smoke-colored when young, soon becoming black.

Tubes black within.

I. B. adusta.

Tubes white within, the mouths black.

2. B. albostygia.

Hymenium pallid when very young, becoming more or less blackish with age.

Pileus $5 \mathrm{~cm}$. or less broad.

3. B. terebrans.

Pileus ro-20 $\mathrm{cm}$. broad.

4. B. subsimulans.

\section{Bjerkandera adusta (Willd.) P. Karst.}

Pileus cespitose-imbricate, decurrent, sometimes effused, conchate, fleshy-tough or corky, somewhat flexible when dry, 2-4 $\times$ 4-8 $\times 0.2-0.4 \mathrm{~cm}$.; surface undulate, indistinctly zonate, especially near the margin, finely tomentose or villose, isabelline with slightly darker markings; margin thin, undulate, sterile, pallid, usually becoming black as though scorched; context fibrous-corky, white, I-3.5 mm. thick; tubes short, I mm. or less long, smoky-white to blackish within, mouths regular, angular, 5-6 to a mm., smoke-colored and pruinose when young, soon becoming grayish-black, edges thin, entire; spores ellipsoidallantoid, 3-5 $\times 1.5-2.5 \mu$.

Common throughout on dead wood.

\section{Bjerkandera albostygia (Berk. \& Curt.) Murrill}

Pileus effused, resupinate to shortly reflexed, $5 \mathrm{~mm}$. thick; surface pallid, finely tomentose, smooth; margin obtuse, baybrown in dried specimens, entire, fertile, finely tuberculose; context thin, less than I mm., white to slightly pallid, homogeneous, somewhat fragile; tubes rather long, slender, stuffed, white to yellowish within, darker near the mouths, $2-3 \mathrm{~mm}$. long, mouths minute, somewhat angular, regular, even, 8 to a $\mathrm{mm}$., edges black, rather thick, obtuse, entire.

Found once on dead wood in Cuba.

\section{Bjerkandera terebrans (Berk. \& Curt.) Murrill}

Pileus subfleshy, thick, flabelliform, convex, $4 \times 4-5 \times \mathrm{I} \mathrm{cm}$., attached by a thick, laterally-compressed, concolorous, pubescent elongation resembling a stipe; surface isabelline or luteous, pubescent-scabrous, azonate, smooth; margin obtuse, entire; context white to isabelline, homogeneous, soft-corky, nearly $\mathbf{I} \mathrm{cm}$. thick; tubes whitish when young, fuliginous in dried specimens, less than I mm., mouths 4 to a mm., edges obtuse, entire.

Found once on dead trees in Cuba. 


\section{BJerkandera subsimulans Murrill}

Pileus explanate, fleshy-tough, sessile, dimidiate or fan-shaped, often attached by a narrow base, 5-10 $\times$ 10-15 $\times 0.3-0.7 \mathrm{~cm}$.; surface smooth, partly glabrous and partly clothed with scanty, flexible hairs; margin thin, acute, broadly sterile, lobed, with a zone of appressed hairs and blackish as though scorched for 5-10 mm.; context fibrous, hard and corky when dry, white to isabelline; tubes $2-5 \mathrm{~mm}$. long, white to fuliginous, mouths angular, irregular, $\mathrm{I}-3$ to a mm., edges thin.

Found once on dead trunks in Cuba.

\section{TRAMETES Fries}

Hymenophore annual, epixylous, sessile; surface anoderm, white, azonate; context white, homogeneous, coriaceous to softcorky; hymenium concolorous, rigid; tubes thin-walled, mouths circular to irregular; spores smooth, hyaline.

Context punky, soft.

Surface entirely white, sometimes discolored with age; pileus less than $5 \mathrm{~cm}$. broad.

Pileus snow-white, dimidiate.

Pileus whitish, opaque, effused-reflexed.

I. T. nivosa.

2. T. jamaicensis.

Surface partly brown or red, especially behind; pileus I0-20 cm. broad.

3. T. cubensis.

Context corky, rather firm.

Pileus effused-reflexed; surface more or less murinous.

Pileus normally expanded; surface not murinous.

Surface white or yellowish.

Context about $5 \mathrm{~mm}$. thick.

Context 2-3 cm. thick.

Surface fulvous tinged with chestnut.

Surface avellaneous to blackish-avellaneous.

Pileus vertically attached, I cm. broad.

4. T. submurina.

5. T. havannensis.

6. T. lignea.

7. T. rutilantiformis.

8. T. jalapensis.

9. T. subscutellatus.

\section{Trametes nivosa (Berk.) Murrill}

Pileus dimidiate, conchate or applanate, 2-3 3 3-5 $\times$ I cm.; surface smooth, azonate, glabrous, snow-white, pale-isabelline in dried specimens; margin thin, concolorous, entire; context soft, punky-corky, white, $5 \mathrm{~mm}$. thick; tubes 2-4 $\mathrm{mm}$. long, slender, white to pallid within, mouths circular, 5 to a mm., edges thin, firm, entire, glistening, white to slightly discolored.

Frequent on dead wood in the West Indies, as well as in South America. 


\section{Trametes Jamaicensis Murrill}

Pileus laterally connate, effused-reflexed, the reflexed portion triangular in section, convex above, concave below, corky, rigid, I $-1.5 \times 2-5 \times 0.5-0.8 \mathrm{~cm}$.; surface slightly uneven, finely tomentose, becoming nearly glabrous, whitish to discolored, opaque, anoderm, azonate; margin subobtuse, entire, fertile, concolorous; context soft-corky, white, 2-3 mm. thick; tubes 2-5 mm. long, rather slender, white within, mouths about 4 to a $\mathrm{mm}$., slightly angular, glistening, edges thin, entire, rigid, white to slightly discolored.

Collected once near Blue Hole, Jamaica, on dead wood.

\section{Trametes cubensis (Mont.) Sacc.}

Plleus large, applanate, dimidiate, corky, rigid or subrigid, comparatively thin, $5^{-10} \times 10-20 \times 0.5^{-1.5} \mathrm{~cm}$.; surface pelliculose, finely tomentose to glabrous, slightly concentrically zoned or furrowed, white to isabelline, latericeous or bay behind or in blutches, or rarely over the whole surface; margin thin, obtuse, sterile, white, entire or undulate; context soft, punky, white to slightly yellowish, 3-10 $\mathrm{mm}$. thick; tubes 2-5 $\mathrm{mm}$. long, slender, firm, corky, mouths very even, regular, circular, 4 to a mm., edges thick, entire, white to ochraceous.

Common throughout on dead logs.

\section{Trametes submurina Murrill}

Pileus broadly effused, reflexed, imbricate, dimidiate, laterally connate, I-2.5 $\times 3-5 \times 0.5^{-1.5} \mathrm{~cm}$.; surface rough, finely tomentose, murinous, sometimes almost white; margin rather thick, undulate, pallid; context white, zonate, fibrous-corky, firm, 5-8 mm. thick; tubes I-2 $\mathrm{mm}$. long, white to slightly yellowish within, mouths circular, quite regular, 4-5 to a mm., edges rather thick, entire, becoming thinner and slightly dentate, white to discolored.

Frequent on old logs in Jamaica and Cuba and occasional in St. John and western Mexico.

\section{Trametes havannensis (Berk. \& Curt.) Murrill}

Pileus simple or subimbricate, dimidiate, convex above, plane or concave below, sessile or umbonate-sessile, $2-3 \times 4^{-5} \times 0.5^{-1}$ cm.; surface zonate, smooth, sometimes sulcate, finely pubescent to glabrous, ochraceous to subfulvous; margin sterile, pubescent, pallid, acute but rather thick; context firm, corky, white, 3-8 
$\mathrm{mm}$. thick; tubes slender, 3-5 $\mathrm{mm}$. long, white within, mouths circular, 4-5 to a mm., edges thick, white, entire, becoming thinner, glistening and subfulvous.

Occasional on dead wood in the West Indies.

\section{Trametes lignea Murrill}

Pileus ungulate, dimidiate, sessile, somewhat laterally connate, slightly decurrent, $3 \times 6 \times 3 \mathrm{~cm}$.; surface smooth, azonate, glabrous, white to isabelline; margin thick, obtuse, entire, concolorous; context woody, white, $2.5 \mathrm{~cm}$. thick; tubes $5 \mathrm{~mm}$. long, white within, mouths angular, irregular, averaging 3 to a mm., edges rather thin, entire, white to slightly discolored.

Found once on dead timber in Nicaragua.

\section{Trametes RUtilantiformis Murrill}

Pileus thick, subtriangular in section, convex above and below, firm but of very light weight, dimidiate, rather broadly attached, $3 \times 4 \times$ I cm.; surface glabrous, slightly sulcate, uneven, with thin cuticle, fulvous tinged with chestnut, margin acute, entire, glabrous, ochraceous; context soft-corky, 2-3 mm. thick, ochraceous; tubes long, slender, ochraceous within, 3-10 mm. long, mouths subcircular, $4-5$ to a mm., edges somewhat glistening, white or whitish, thin, entire, corky; spores oblong-ellipsoid, 4-5 $\times 1.5^{-2 \mu}$.

Collected once on dead wood in British Honduras.

\section{Trametes Jalapensis Murrill}

Pileus of medium size, dimidiate to flabelliform, sessile, corky, scarcely flexible, imbricate, $2-4 \times 3-7 \times 0.5^{-1} \mathrm{~cm}$.; surface pelliculose, glabrous, azonate, smooth, avellaneous to blackish-avellaneous, margin acute or slightly obtuse, sterile, entire, concolorous; context punky, homogeneous, white with a fa:nt yellowish or rosy tint, $3-7 \mathrm{~mm}$. thick; tubes $2 \mathrm{~mm}$. long, rigid, corky to woody, discolored-whitish within, mouths circular, 2-3 to a mm., edges rather thick, entire or slightly dentate, pale-umbrinous.

Collected once on a railway tie near Jalapa, Mexico.

\section{Trametes subscutellatus Murrill}

Pileus tough to rigid, concave below, convex above, vertically attached, circular in outline, I cm. broad, $2 \mathrm{~mm}$. thick; surface nearly smooth, with a thin pellicle, ochraceous except at the vertex, where it is latericeous to castaneous; margin subobtuse or 
acute, deflexed, white, entire; context white, firm, fibrous, homogeneous; tubes I $\mathrm{mm}$. long, stramineous within and without, mouths regular, $4^{-5}$ to a $\mathrm{mm}$., circular to slightly angular, glistening, edges obtuse, entire.

Collected once between Moneague and Union Hill, Jamaica, on dead wood.

\section{Io. EARLIELLA Murrill}

Hymenophore medium to large, annual, epixylous, semiresupinate, thin and dry but rigid; surface pelliculose, glabrous, zonate, more or less reddish-brown in color; context white, coriaceous, zonate; hymenium flesh-colored, tubes medium, irregular, becoming thin-walled; spores smooth, hyaline.

\section{EARLIElla CoRrugata (Pers.) Murrill}

Pileus annual, often reviving, semiresupinate, laterally extended, conchate, imbricate, $3^{-6} \times 5^{-1} 5 \times 0.2-0.5 \mathrm{~cm}$.; surface thinly encrusted, glabrous, rugose, dark-reddish-brown behind, or with a white marginal band $3^{-12} \mathrm{~mm}$. in width; margin tumid, at length thin, undulate or lobed, fertile; context concentrically zonate; tubes $2-3 \mathrm{~mm}$. long, 2-4 to a mm., white within, the mouths reddish-flesh-colored fading to white, dissepiments at first thick, at length becoming thin and irregular with wavy edges; spores ellipsoid, 3-4 $\times 5^{-6} \mu$.

Common throughout on dead hardwood trunks.

\section{RIGIDOPORUS Murrill}

Hymenophore annual, at times reviving, epixylous, sessile, dimidiate to circular, simple or imbricate; surface pelliculose, multizonate, margin thin, incurved when dry; context thin, white, fleshy-corky, very rigid when dry; tubes minute, regular, light-colored, mouths usually pruinose when young; spores smooth, hyaline.

Pileus effused, very narrowly reflexed.

I. R. microstomus.

Pileus circular, fixed at the center.

2. $R$. evolutus.

Pileus normally expanded, dimidiate or fan-shaped, sometimes decurrent.

Tubes very short, $0.5-1.5 \mathrm{~mm}$. long.

Pileus latericeous to bay with isabelline lines; tubes 6-7 to a $\mathrm{mm}$.

Pileus bay to blackish; tubes ro to a $\mathrm{mm}$.
ubes rather slender, 2-4 mm. long.

3. $R$. substereinus.

Edges very obtuse.

4. R. Liebmanni.

Edges thin.

5. $R$. contrarius.

6. R. surinamensis. 


\section{Rigidoporus microstomus (Berk. \& Curt.) Murrill}

Pileus effused, laterally connate, very shortly reflexed, $\mathrm{I}-4 \times$ I-I.5 $\times 0.2-0.4 \mathrm{~cm}$., the reflexed portion very narrow, latericeous, finely pubescent to glabrous, faintly zonate, with a thin, undulate or slightly lobed, yellowish-white margin; context very thin, white, fibrous; tubes long and slender, 2-3 mm., white within, mouths minute, 7 to a mm., ochraceous or flesh-colored, edges obtuse, entire.

Found once on dead wood in Cuba.

2. Rigidoporus evolutus (Berk. \& Curt.) Murrill

Pileus fleshy-tough, circular to flabelliform, I-2.5 cm. broad, I-2 mm. thick; surface radiate-rugose, tomentose to resinousglabrous, latericeous with ochraceous blotches, black behind; margin thin, acute, undulate to slightly lobed, ochraceous; context very thin, pallid, fibrous; tubes pallid, $\mathbf{I}-1.5 \mathrm{~mm}$. long, mouths minute, 7 to a mm., subcircular, edges white to yellowishdiscolored, thin, dentate.

Found once on dead trees in Cuba, and once on dead wood in the mountains of Porto Rico.

\section{RIGIDOPORUS SUBSTEREINUS Murrill}

Pileus thin, coriaceous, inflexed when dry, dimidiate, attached by a narrow base, applanate, $\mathrm{I}-3 \times \mathrm{I} .5-5 \times 0 . \mathrm{I}-0.2 \mathrm{~cm}$.; surface smooth, shining, glabrous, conspicuously zonate, radiate-rugose, latericeous to bay with isabelline lines, much resembling a Stereum; margin very thin, smooth, cremeous, broadly sterile below, sharply inflexed on drying; context exceedingly thin, white, fibrous; tubes minute, $0.5-1.5 \mathrm{~mm}$. long, white to pallid within, mouths very even, regular, angular, inconspicuous, 6-7 to a mm., edges thin, entire, whitish-pruinose to ochraceous or pale-flesh-colored.

Occasional on dead sticks in woods in Cuba, British Honduras, and eastern Mexico.

\section{Rigidoporus Liebmanni (Fries) Murrill}

Pileus fleshy to woody, thin, flabelliform or obovate, convex, I.5-2 $\times 2-3 \times 0.2-0.4 \mathrm{~cm}$.; surface glabrous, bay or blackish, often rugose behind; margin thin, entire, pallid, sterile, inflexed when dry; context very thin, white, fibrous, brown and very fragile in type specimens; tubes slender, I-I.5 $\mathrm{mm}$. long, white to pallid within, mouths whitish-pruinose to yellowish-brown, 
subcircular, inconspicuous, even, regular, to to a mm., edges thin, entire; spores ovoid.

Frequent to common on dead wood in Mexico, Cuba, and Porto Rico.

\section{Rigidoporus contrarius (Cooke) Murrill}

Pileus attached by the vertex, subcircular to dimidiate, applanate, fleshy-corky, $3-6 \times 5-1$ I $\times 0.3-0.5 \mathrm{~cm}$.; surface ochraceous, soon becoming brown, zonate, tomentose, at length glabrous, slightly concentrically sulcate; margin thin, entire or undulate, sterile, ochraceous, easily bruised; context thin, fibrous-corky, pallid, I-3 mm. thick; tubes pallid, 2-4 mm. long, mouths circular, regular, white to discolored, 5 to a mm., edges very obtuse, entire, glistening.

Found a few times on dead trunks in Cuba.

\section{Rigidoporus surinamensis (Miq.) Murrill}

Pileus imbricate-multiplex, laterally connate, dimidiate or reniform, fleshy-corky, convex or applanate, $2-3 \times 2.5-5 \times 0.3^{-}$ $0.6 \mathrm{~cm}$.; surface smooth or rugulose, pruinose to glabrous, isabelline to latericeous; margin acute, thin, inflexed, entire or undulate, often obtuse with age; context very thin, white, fibrous, I-2 mm. thick; tubes white, 2-4 mm. long, slender, mouths minute, circular to angular, regular, even, 6 to a mm., edges thin, entire, white to pallid, becoming discolored with age; spores globose, 3-4.5 $\mu$.

Common throughout on water-soaked hardwood trunks.

\section{I2. PORODISCULUS Murrill}

Hymenophore small, annual, tough, epixylous, erumpent from the lenticels of dead branches; stipe attached to the vertex of the pileus, usually curved at maturity; context white, fibrous; tubes cylindric, short, one-layered, mouths constricted; spores globose, smooth, hyaline.

\section{Porodisculus Pendulus (Schw.) Murrill}

Pileus very small, turbinate-cup-shaped, attached at the vertex, soon decurved and pendant, gregarious, erumpent from the lenticels of dead branches, $\mathrm{I}-2 \mathrm{~mm}$. broad, 3-5 mm. long; surface anoderm, azonate, smooth, umbrinous, uniformly covered with a brown powder, often ashy-white with age; margin inflexed, concolorous, sterile; context white, fibrous, very thin; tubes very 
short, annual, white within, mouths circular, constricted, white, pruinose, becoming concolorous, $6-7$ to a mm., edges entire; spores globose, $4 \mu$; stipe $2 \mathrm{~mm}$. or less in length, vertically attached, gradually expanding into the pileus, which it resembles in surface and context.

Found in Nicaragua.

\section{HEXAGONA Pollini}

Hymenophore small, annual, epixylous, flabelliform to reniform, rarely circular, stipitate, the stipe sometimes much reduced; surface smooth or tessellate, margin thin; context thin, white, fibrous, fleshy to tough, usually fragile when dry; hymenium of radiating rows of large, thin-walled, hexagonal tubes, usually radially elongate; spores smooth, hyaline.

Tubes unequally hexagonal, the radial walls longer.

Pileus white or nearly so.

Surface of pileus not distinctly tomentose.

Stipe 5-10 $\mathrm{mm}$. in diameter.

Tubes $\mathrm{I} \mathrm{mm}$. in length.

Tubes 3-6 mm. in length.

I. H. princeps.

2. $H$. pseudoprinceps.

Stipe $\mathrm{I}-3 \mathrm{~mm}$. in diameter.

Pileus less than $3 \mathrm{~cm}$. broad.

Tubes flesh-colored, $2 \mathrm{~mm}$. wide.

Tubes pallid, I mm. wide.

Pileus more than $3 \mathrm{~cm}$. broad.

Margin entire, often pellucid.

Tubes I-2.5 mm. wide.

Tubes much smaller, only $0.5 \mathrm{~mm}$. wide.

Margin ciliate or denticulate.

Surface minutely checkered.

Surface not checkered.

3. H. Maxoni.

4. H. floridana.

5. H. daedalea.

6. H. daedaleiformis.

7. H. tessellatula.

8. H. fragilis.

Surface of pileus distinctly tomentose.

Context thin, translucent.

9. H. reniformis.

Context thick, opaque.

Surface tessellate; stipe distinct.

Surface not tessellate; stipe a mere tubercle.

Pileus sulfur-colored throughout.

Pileus pale-avellaneous-isabelline, becoming bay on drying.

Io. H. subcaperata.

II. $H$. caperata.

I2. H. sulfurea.

13. H. motzorongensis.

Pileus purple or brown.

Pileus purple or purplish-brown; stipe lateral or eccentric.

Pileus subcircular; stipe eccentric; tubes 2.5 $\mathrm{mm}$. long.

14. H. subpurpurascens.

Pileus spatulate; stipe lateral; tubes $1.5 \mathrm{~mm}$. long.

15. H. purpurascens. 
Pileus brown; stipe distinctly central.

Margin entire.

Margin ciliate.

Tubes equally hexagonal.

Pileus purple.

Pileus yellow or brown.

Tubes merulioid.

Tubes of normal length.
I6. $H$. portoricensis.

17. H. hondurensis.

18. H. indurata.

I9. H. brunneola.

20. H. cucullatc.

I. Hexagona PRinceps (Berk. \& Curt.) Murrill

Pileus reniform, convex, $9 \times 6 \times 0.4-0.6 \mathrm{~cm}$.; surface fulvous, finely tomentose, smooth; margin thin, entire or undulate, inflexed when dry; context fleshy-tough, white, opaque; tubes decurrent, fuscous when dry, I $\mathrm{mm}$. long, 4-6-angled, mouths I $\times$ I.5 mm., edges thin, dentate; spores ellipsoid; stipe lateral, tapering downward, fulvous, velvety, $3 \mathrm{~cm}$. long, I-5 cm. thick.

Found once on dead wood in Cuba and once in Costa Rica.

\section{HeXagona PSEUdoprinceps Murrill}

Pileus reniform, convex, depressed behind, $3.5 \times 4.5 \times 0.5^{-I}$ $\mathrm{cm}$; surface glabrous, delicately radiate-striate, discolored on drying; margin thick, entire, strongly inflexed when dry; context fleshy-tough, white, opaque; tubes decurrent, white, discolored on drying, 3-6 mm. long, 4-6-angled, mouths $\mathrm{I} \times 2 \mathrm{~mm}$., sometimes confluent in age, edges thin, entire or slightly toothed; spores ellipsoid; stipe eccentric to lateral, concolorous, glabrous or slightly hispid, $5 \mathrm{~mm}$. long, $7 \mathrm{~mm}$. thick.

Found once on dead wood at Carmelita, Porto Rico.

\section{Hexagona Maxoni Murrill}

Pileus irregularly reniform, applanate, $1-2 \times 1.5-3 \times 0.1 \mathrm{~cm}$.; surface glabrous, tessellate, straw-colored on drying; margin thin, denticulate, undulate to lobed; context fleshy-tough, membranous, white, opaque; tubes slightly decurrent, darker than the pileus, with a tinge of flesh-color, $0.5-1.5 \mathrm{~mm}$. long, 4-6-angled, mouths $1.5 \times 2-3 \mathrm{~mm}$., smaller near the margin, edges thin, entire or undulate; spores ellipsoid; stipe lateral, tapering, concolorous, glabrous, $2-3 \mathrm{~mm}$. long, $\mathrm{I}-2 \mathrm{~mm}$. thick, not umbilicate above.

Found once on dead wood in Guatemala.

\section{Hexagona floridana Murrill}

Pileus flabelliform, applanate, depressed behind, $2 \times 2.5-3 \times$ $0.2 \mathrm{~cm}$.; surface finely hispid, pure-white, becoming straw-colored 
on drying, faintly radiate-striate; margin slightly undulate, tessellate at times, fringed with numerous slender cilia; context very thin, white, membranous, wholly translucent; tubes decurrent, white, becoming ochraceous, I.5-2 mm. long, normally hexagonal, mouths $0.5^{-1} \times 2-3 \mathrm{~mm}$., not radially confluent, edges thin, fimbriate; spores ellipsoid, $9 \times 4 \mu$; stipe lateral, subequal, scutate at the base, concolorous, hispid, 2-5 mm. long, I-2 mm. thick, always umbilicate above.

Found on old logs in rich woods at Miami, Florida.

\section{Hexagona daedalea (Link) Murrill}

Pileus spatulate-obovate to reniform, applanate, usually attenuate behind, $4-8 \times 2-6 \times 0.1-0.3 \mathrm{~cm}$.; surface white when fresh, radiate-striate, finely tomentose to glabrous, hispid behind, tessellate near the margin, which is thin, of ten pellucid, undulate, at times fimbriate, lobed or fissured with age; context fleshy, fragile when dry, white, often partially translucent; tubes decurrent, concolorous, I-2 $\mathrm{mm}$. long, 4-6-angled, mouths very variable in size, $1-2.5 \times 3-7 \mathrm{~mm}$., smaller near the margin, edges thin, but apparently firm, at length splitting into irregular teeth; spores ellipsoid, 9-12 $\times 4^{-5} \mu$; stipe lateral, concolorous, hispid-tomentose, $0.5^{-1} \mathrm{~cm}$. long, 2-5 mm. thick, usually dilated at the base.

Common on dead wood throughout.

\section{Hexagona daedaleiformis Murrill}

Pileus fan-shaped to reniform, sometimes slightly attenuate behind, subapplanate, plane or concave below, long-stipitate, $2-3$ $\times 3-4 \times 0.1-0.2 \mathrm{~cm}$.; surface pallid, pale-fulvous when dry, radiate-striate and finely areolate over most of the surface, minutely hispid; margin entire or undulate, thin, concolorous, inflexed on drying; context thin, white, fibrous; tubes pallid within, scarcely over I mm. deep, decurrent; mouths regular, pallid to isabelline, somewhat glistening, small, $0.5 \mathrm{~mm}$. wide, $1 \mathrm{~mm}$. or more long; edges very thin, soon becoming fimbriate; stipe lateral, concolorous or paler, glabrous, $1.5^{-2} \mathrm{~cm}$. long, $2-3 \mathrm{~mm}$. thick at the base, increasing to 5 or $6 \mathrm{~mm}$. above, furrowed on the back, subcylindric below.

Collected once on dead wood in Mexico.

\section{Hexagona tessellatula Murrill}

Pileus flabelliform, convex, depressed behind, $\mathrm{I}-3 \times 2-4 \times 0.2$ $\mathrm{cm}$.; surface delicately and closely tessellate, finely hispid, becom- 
ing glabrous, white, yellowish when dry; margin thin, denticulate, slightly incurved when dry, frequently brown and hygrophanous; context white, membranous, translucent; tubes decurrent, white, $2 \mathrm{~mm}$. long, 4-6-angled, mouths $\mathrm{I} \times 3 \mathrm{~mm}$., at length radially confluent, edges thin, lacerate; spores ellipsoid, 2-guttulate, $3 \times$ ro $\mu$; stipe lateral, short, slightly enlarged below, concolorous, hispid, 3-5 mm. long, 2-3 mm. thick, usually umbilicate above.

Found a few times on dead wood in Cuba, Santo Domingo, and Jamaica.

\section{HeXagona Fragilis Murrill}

Pileus flabelliform, convex, depressed behind, $2-3 \times 2-4 \times$ $0.1-0.3 \mathrm{~cm}$.; surface densely hispid, especially behind, faintly radiate-striate, pure-white, becoming straw-colored on drying; margin quite thick for the genus, nearly regular in outline, usually inflexed when dry, partly hygrophanous at times, beset with short, fimbriate, fugacious hairs; context thin, white, partially translucent, quite fragile when dry; tubes decurrent, white, 2-3 mm. long, 4-6-angled, mouths I $\times 4 \mathrm{~mm}$., becoming radially confluent and gill-like, edges thin, lacerate, the divisions fimbriate; spores ellipsoid, 4-5 $\times$ ro $\mu$; stipe lateral, subequal, concolorous, hispid, short, $5 \mathrm{~mm}$. long, $2.5 \mathrm{~mm}$. thick, rarely umbilicate above.

Frequent on dead wood in Cuba, Jamaica, and Grenada.

\section{HeXagona RENIFORMis Murrill}

Pileus reniform, slightly convex, $2-4 \times 2-4.5 \times 0.2-0.4 \mathrm{~cm}$.; surface densely tomentose, tessellate near the margin, pallid, yellowish-brown on drying; margin undulate, rather thick, entire; context fleshy-tough, membranous, white, partially translucent; tubes not decurrent, white, becoming yellowish-brown on drying, I-2 mm. long, 4-6-angled, mouths $1.5 \times 2-2.5 \mathrm{~mm}$., edges entire; spores ellipsoid; stipe lateral, equal, concolorous or lighter, tomentose, $0.5-1 \mathrm{~cm}$. long, $2.5-5 \mathrm{~mm}$. thick, not umbilicate above.

Collected once on decayed deciduous wood in hammocks near the Homestead Trail, Florida, and four times at Motzorongo, Mexico.

\section{io. Hexagona subcaperata Murrill}

Pileus flabelliform, convex, somewhat attenuate and depressed behind, 4-6 $\times 55^{-8} \times 0.3-0.5 \mathrm{~cm}$.; surface distinctly tomentose, pale-ochraceous, rather coarsely checkered; margin abruptly acute, 
entire, often brownish and much deflexed when dry; context white, thin, opaque; tubes decurrent, white when fresh, discolored on drying, 3-4 $\mathrm{mm}$. long, mouths large and angular, I-I.5 $\times 2-2.5 \mathrm{~mm}$., often radially confluent with age, much contorted on drying, edges thin, lacerate, the divisions fimbriate; spores ellipsoid; stipe lateral, slightly tapering downward, lighter than the pileus in dried specimens, minutely tomentose, $\mathbf{I} \mathbf{~ c m}$. long, $5 \mathrm{~mm}$. thick, neither umbilicate above nor scutate below.

Found a few times on dead logs in Jamaica and western Cuba.

\section{Hexagona caperata (Pat.) Murrill}

Pileus convex, attenuate behind; surface striate, pallid to reddish-brown, with a conspicuous covering of short, grayish hairs, which partly disappear with age; margin more or less incised or lobed, deflexed, neither ciliate nor tessellate; context fleshy, white; tubes deep, white, angular, with large, soft, fleshy, fimbriate dissepiments; spores ovoid-cylindric, 10-12 $\times 5 \mu$; stipe wanting, or a mere rounded lateral tubercle.

Found once in Guadeloupe on a dead trunk of Byrsonima.

\section{I2. Hexagona sulfurea Murrill}

Pileus sulfureous throughout, sessile or attached by a much reduced stipe, very irregular, usually fan-shaped, conchate, $I \times$ I $X$ o.I cm.; surface smooth, glabrous, margin lobed and usually splitting deeply with age, much inflexed on drying; context thin, white, fibrous, fragile when dry; tubes shallow, angular, elongate, about $0.5^{-1} \mathrm{~mm}$. broad, I-2 $\mathrm{mm}$. long, edges rather thick, entire or dentate.

Collected once on dead wood in a moist virgin forest at Motzorongo, near Cordoba, Mexico.

\section{I3. HeXagona motzorongensis Murrill}

Pileus minute, thin, dimidiate, depressed above, convex below, attenuate behind, $2 \mathrm{~cm}$. broad; surface pale-avellaneous-isabelline, becoming bay on drying, tessellate, minutely hispidfibrillose; context thin, white, fragile; tubes white, of medium size for the genus, I-2 mm. long, angular; edges thin, fimbriate, lacerate with age; stipe reduced, furrowed above, hispidtomentose, pallid, I-2 mm. long, I mm. thick.

Collected twice on dead wood in a moist virgin forest at Motzorongo, near Cordoba, Mexico. 


\section{Hexagona subPuRPurascens Murrill}

Pileus subcircular, convex, depressed at the center, I-I.5 $\times$ I-I.5 $\times 0.1-0.2 \mathrm{~cm}$.; surface reddish-brown with purple areoles, subpruinose to glabrous; margin very thin, entire, strongly inflexed on drying; context fleshy-tough, membranous, white, opaque; tubes decurrent, white, I-I.5 mm. long, 4-6-angled, mouths I $\times 1.5 \mathrm{~mm}$., edges thin, entire; spores ellipsoid; stipe eccentric, slightly tapering, white, $0.5^{-1} \mathrm{~cm}$. long, I. $5 \mathrm{~mm}$. thick.

Found once on dead sticks in woods at Moore Town, Jamaica.

\section{Hexagona purpurascens (Berk. \& Curt.) Murrill}

Pileus spatulate to flabelliform, I.5-2.5 $\times$ I-4 $\times 0 . \mathrm{I}-0.3 \mathrm{~cm}$.; surface lurid-purple, glabrous; margin thin, entire, incurved when dry; context fleshy-tough, white, opaque; tubes decurrent to the base of the stipe, lighter than the pileus, $2 \mathrm{~mm}$. long, 4-6-angled, mouths I-I.5 $\times 2-2.5 \mathrm{~mm}$., edges thin, becoming lacerate; spores ellipsoid; stipe lateral, equal, more or less hispid, $4 \mathrm{~mm}$. long, I-2 mm. thick.

Found a few times on tree trunks in Cuba.

\section{i6. HeXagona PORTORICENSIS Murrill}

Pileus centrally stipitate, circular, deeply umbilicate, $4 \times 0.3$ $\mathrm{cm}$.; surface subglabrous, umbrinous, the center concolorous; margin entire, not very thin, much inflexed when dry; context white, I mm. thick, opaque; tubes somewhat decurrent, white, $2 \mathrm{~mm}$. long, 4-6-angled, mouths I $\times 3 \mathrm{~mm}$., smaller and more regular near the margin, edges thin, fimbriatulate; spores ellipsoid, 2-guttulate, 8-Io $\times 3-5 \mu$; stipe central, compressed, slightly tapering downward, subconcolorous, minutely tomentose, $2 \mathrm{~cm}$. long, $4 \mathrm{~mm}$. thick.

Found once on dead wood in Porto Rico.

\section{I7. Hexagona hondurensis Murrill}

Pileus centrally stipitate, circular, slightly umbilicate, $2-4 \times$ $0.05 \mathrm{~cm}$.; surface faintly radiate-striate, subglabrous, fulvous, the center fuliginous; margin thin, regular, tessellate, inflexed when dry, fringed with numerous short, fugacious cilia; context white, fibrous, $0.25 \mathrm{~mm}$. thick, translucent near the margin; tubes adnate, white, $0.3 \mathrm{~mm}$. long, hexagonal, radially elongate, mouths I $\times 2 \mathrm{~mm}$., much smaller near the margin, edges thin, subentire; spores ellipsoid, $9 \times 3.5 \mu$; stipe central, equal, concolorous, pruinose to glabrous, $2-3 \mathrm{~cm}$. long, $2 \mathrm{~mm}$. thick.

Found once on dead logs near the coast in Honduras. 


\section{I8. Hexagona indurata (Berk.) Murrill}

Pileus reniform, slightly convex, attached by the vertex, $2.5 \times 3 \times 0.2-0.3 \mathrm{~cm}$.; surface liver-colored, glabrous, usually tessellate; margin rather thick, entire; context fleshy-tough, white, opaque; tubes lighter than the pileus, subhexagonal, $\mathbf{I}-\mathbf{I} .5 \mathrm{~mm}$. long, mouths I $\mathrm{mm}$. or less in diameter, edges entire to toothed; spores ellipsoid.

Found once on dead wood in Santo Domingo.

\section{Hexagona brunneola (Berk. \& Curt.) Murrill}

Pileus flabelliform, wedge-shaped behind, $2.5 \times 2.5 \times 0 . \mathrm{I}-0.2$ $\mathrm{cm}$.; surface brown, glabrous, rugulose when dry; margin thin, undulate or lobed; context membranous, white, opaque; tubes discolored, very short, much less than a mm., subhexagonal, mouths I mm. in diameter, edges entire; spores ellipsoid; stipe spurious or a mere lateral disk.

Found once on dead wood in Cuba.

\section{Hexagona cucullata (Mont.) Murrill}

Pileus reniform, convex, umbonate-sessile, $3-6 \times 6-8 \times 0.2$ $\mathrm{cm}$.; surface glabrous, often radiate-striate, cream-colored to ochraceous, plane or marked with two or three broad undulations from center to margin, which is thin, entire, irregularly undulate or lobed and deflexed when dry; context fleshy-tough, white, $0.5 \mathrm{~mm}$. thick; tubes ochraceous to dark-fulvous, hexagonal, not radially elongate, very variable in size, $1.5-3.5 \mathrm{~mm}$. long, I-3 mm. wide, edges thin, finely denticulate; spores ellipsoid, I $4 \times 7 \mu$; stipe a mere scutate disk nearly a centimeter in breadth.

Frequent on dead wood throughout most of the region.

\section{I4. MICROPORELLUS Murrill}

Hymenophore thin, annual, epixylous, usually flabelliform, stipitate, the stipe variously attached and sometimes much reduced; surface anoderm, multizonate; context thin, white, fibrous, rigid and fragile when dry; tubes very minute, regular, thin-walled, fragile when dry; spores smooth, hyaline.

Pileus white or pale-brown, unchanging.

Tubes entire.

Tubes lacerate.

Pileus ochraceous or sordid, becoming bay or black with age or on drying.

Pileus ochraceous, becoming bay.

Pileus sordid, becoming black.
I. M. dealbatus.

2. M. unguicularis.

3. M. porphyritis.

4. M. holotephrus. 


\section{Microporellus dealbatus (Berk. \& Curt.) Murrill}

Pileus thin, coriaceous, slightly flexible but easily broken, flabelliform or spatulate, conchate, $2-6 \times 3-7 \times 0.1-0.2 \mathrm{~cm}$.; surface finely tomentose to glabrous, hirtose behind, radiatestriate, dealbate, the zones often light-fulvous; margin very thin, sterile, sericeous, undulate to lobed or cleft, inflexed and often splitting when dry; tubes white to isabelline within, scarcely a mm. in length, mouths minute, angular, 8-10 to a $\mathrm{mm}$., edges thin, entire, glistening, whitish when young, becoming discolored; stipe variable, often wanting, $0-7 \mathrm{~cm}$. long, 2-7 mm. thick, scutate at the base, expanding into the pileus, laterally attached, rarely eccentric, usually compressed, with surface and substance resembling that of the pileus.

Common throughout on dead wood. The form originally described as $P$. mutabilis is by far the most common.

\section{Microporellus unguicularis (Fries) Murrill}

Pileus thin, coriaceous, reniform or flabelliform, attached by an attenuate base, $2-3 \mathrm{~cm}$. broad, I-3 mm. thick; surface uniformly ochroleucous, very smooth, concentrically striate, radiate-lineate; margin acute, incurved when dry; context very thin, less than I mm., fibrous, somewhat fragile, watery-white; tubes $0.5^{-1} \mathrm{~mm}$. long, pallid to yellowish, mouths irregular, angular, $2-4$ to a mm., edges white to pallid, thin, fimbriatedentate, at length lacerate.

Found once on dead trunks in Mexico.

\section{Microporellus PORPHYRITIS (Berk.) Murrill}

Pileus thin, coriaceous, flabelliform, 4-5 $\times 5^{-6} 60.1-0.3 \mathrm{~cm}$.; surface pelliculose, ochraceous to light-bay, zonate, glabrous; margin thin, ochraceous, undulate; context fibrous-corky, I-I.5 $\mathrm{mm}$. thick, pallid; tubes short, less than I mm., mouths minute, 5-6 to a mm., pallid to discolored, very regular, subangular, edges thin, entire, at first obtuse, becoming acute; stipe variable, short, slightly lighter than the pileus, 5-10 mm. long, 5-8 mm. thick, resembling the pileus in surface and substance.

Occasional on dead wood in Cuba and western Jamaica, as well as in Brazil.

\section{Microporellus holotephrus (Berk. \& Curt.) Murrill}

Pileus thin, coriaceous, fan-shaped, attenuate behind, laterally attached, $2-5 \times 2-6 \times 0.1 \mathrm{~cm}$.; surface conspicuously scabrous, 
tomentose, zonate, radiate-lineate, brown, tinged with winecolor, becoming very blackish and opaque in type specimens; margin extremely thin, even or undulate; context tough, fibrous, very thin, discolored in type specimens; tubes extremely short, angular, 8 to a mm., edges thin, entire, white to discolored, nearly black in type specimens; stipe laterally attached, compressed, sometimes wanting, rarely over $2 \mathrm{~cm}$. in length or $5 \mathrm{~mm}$. in breadth, expanding into the pileus, which it resembles in surface and substance.

Occasional on dead wood in Cuba; also reported from Guiana.

\section{POLYPORUS (Micheli) Paulet}

Hymenophore annual, epixylous, small and simple, very rarely large and compound; stipe central, eccentric or lateral, much reduced at times in a few species, often partly or wholly brown or black; surface usually smooth, the margin at times ciliate; context white or yellowish, fibrous, tough to corky; hymenium porose, at times alveolate; spores smooth, hyaline.

Stipe pallid or light-brown, centrally attached, not darker than the pileus.

Margin of pileus devoid of cilia.

Pileus beset, especially near the margin, with hydnoid processes.

I. P. hydniceps.

Pileus plainly villose, tomentose or scabrous, often becoming glabrous with age.

Pileus scabrous, irregular, umbrinous, margin involute; stipe scabrous; tubes small, 4 to a mm., dissepiments dentate.

Pileus villose or tomentose, becoming virgate from the rupture of the cuticle.

Pileus minutely tomentose or glabrous from the first.

Hymenophore goblet-shaped; pileus less than 3 $\mathrm{cm}$. broad, shallowly depressed at the center; stipe long, striate, expanding into the pileus.

Hymenophore trumpet-shaped; pileus $5-\mathrm{ro} \mathrm{cm}$. broad, deeply infundibuliform; stipe $3 \mathrm{~cm}$. long, pallid, puberulent.

Hymenophore not as above.

Pileus minute, $2 \mathrm{~mm}$. in diameter, umbilicate, margin involute; pores alveolar.

Pileus large, ro $\mathrm{cm}$. or more in diameter.

Pileus of medium size, $2-3 \mathrm{~cm}$. in diameter.

2. P. scabriceps.

3. P. virgatus.

4. P. Tuba.

5. P. cyathiformis.

6. P. Acicula.

7. P. discoideus.

8. P. obolus.

Margin of pileus ornamented with cilia, which often disappear with age.

Tubes alveolar.

Margin of pileus finely hispid, broadly sterile below.

9. P. aemulans. 
Margin of pileus strigose, fertile below.

Tubes not alveolar.

Tubes very irregular; stipe usually thick and polished; pileus tough, umbilicate, yellowishwhite with brown marginal band; cilia short, fugacious.

II. P. variiporus.

Tubes fairly regular; stipe slender, not polished; plants rather delicate; cilia variable in form and persistence.

Pileus opaque, not translucent, $\mathrm{I}-4 \mathrm{~cm}$. in diameter; cilia long, of uncertain duration; plants mostly cespitose.

Io. $P$. arcularius.

Pileus very thin, more or less translucent, I-2 $\mathrm{cm}$. in diameter; cilia short, slender, fugacious; plants not cespitose.

Stipe wholly or partly black or fuliginous, variously attached, usually darker than the pileus.

Pileus finely tomentose, drab-colored, with reddishbrown spots, small, circular; tubes rounded, minute. Pileus not as above.

Tubes reaching $0.5^{-1} \mathrm{~mm}$. or more in diameter.

Stipe short, less than I cm. long; pileus normal.

Stipe usually very long, $\mathrm{I}-\mathrm{r} 5 \mathrm{~cm}$.; pileus sometimes aborted, resembling a $X$ ylaria.

Tubes much smaller, 4-ro to a mm.

Surface light-colored, isabelline to pale-umbrinous.

Stipe central, or excentric; species confined to temperate regions.

Stipe lateral; species confined to tropical regions.

Surface dark-colored, bright-bay to almost black.

Stipe central; pileus proliferous at times, surface glabrous; species confinèd to tropical and South Ámerica.

Stipe horizontal, usually lateral or excentric, rarely central in temperate species.

Stipe $2-6 \mathrm{~cm}$. long; pileus glabrous, pale-fuliginous.

Stipe $0.3^{-1} \mathrm{~cm}$. long; pileus bay or chestnut to blackish.

Surface glabrous.

Surface marked with rows of serrated black squamules. Stipe obsolete; pileus latericeous, spotted.

12. P. Tricholoma.

13. P. Cowellii.

I4. P. maculosus.

15. P. Wrightii.

16. P. marasmioides.

17. P. elegans.

18. P. subelegans.

19. P. diabolicus.

-

20. P. Marbleae.

21. P. Blanchetianus.

2x. $P$. scabellus.

23. $P$. praeguttulatus.

\section{Polyporus hydniceps Berk. \& Curt.}

Pileus cup-shaped or irregularly broken up into flabelliform lobes, 3-4 $\times 0.1-0.2 \mathrm{~cm}$.; surface light-brown to bay, adorned with very prominent cylindric or subpyramidal processes; margin thin, irregular, undulate or lobed, inflexed; context pallid, fleshy, rigid and fragile when dry, about I mm. thick; tubes 
decurrent, pallid to ochraceous, short, mouths subelliptic or circular, becoming angular, 4-6 to a mm., edges entire to dentate; spores smooth, hyaline; stipe central or eccentric, short, thick, usually reticulate, brown to blackish, fleshy, $2 \mathrm{~cm}$. long, 5-15 mm. thick.

Found a few times on dead wood in Cuba.

\section{Polyporus sCABriceps Berk. \& Curt.}

Pileus irregularly circular in outline, slightly convex, 3-5 $\times 0.3$ $\mathrm{cm}$.; surface umbrinous, minutely and evenly scabrous, appearing spuriously porose; margin irregular, involute, 3-4 $\mathrm{mm}$. of it being rolled inward; context soft-corky, pale-yellowish-brown; tubes decurrent, pallid, dark-yellowish-brown when dry, mouths irregular, 4 to a mm., edges thin, dentate; stipe central, short, even, lighter than the pileus, scabrous, tough, $2 \mathrm{~cm}$. long, 5-6 mm. thick.

Found once on dead wood in Cuba.

\section{Polyporus virgatus Berk. \& Curt.}

Pileus circular, depressed at the center, $3.5-6 \times 0.2-0.3 \mathrm{~cm}$.; surface rufous when dry, tomentose to glabrous, at length virgate from the rupture of the cuticle; margin thin, acute, inflexed when dry; context firm, fibrous, pale-brown, I-2 $\mathrm{mm}$. thick; tubes decurrent, fragile, dark-yellowish-brown when dry, I-I.5 mm. long, mouths angular, somewhat irregular, 2-3 to a mm., slightly concentrically confluent, edges thin, conspicuously dentate; stipe central, nearly equal, concolorous or darker at the base, smooth like the pileus, firm and tough, $1.5-2.5 \mathrm{~cm}$. long, 5-10 $\mathrm{mm}$. thick.

Found a few times on dead wood in Cuba, once in Costa Rica, and once at Hogtown, Florida.

\section{Polyporus Tuba Berk. \& Curt.}

Pileus cup-shaped, like a Peziza, with a very long stipe, and very thick for its size, I-I.5 $\times 0.5^{-I} \mathrm{~cm}$.; surface white, yellowish-brown when dry, glabrous, smooth or radiate-rugose; margin abruptly acute, entire, slightly revolute when dry; context pallid, firm, not elastic, $5 \mathrm{~mm}$. or more thick; tubes decurrent, short, yellowish, mouths small, 4 to a mm., edges thin, at length elongate; stipe slender, central, dilated above, concolorous, rough, striate, tough, $3-4 \mathrm{~cm}$. long, 2-4 mm. thick.

Found once on dead wood in Cuba. 


\section{Polyporus cyathiformis Lév.}

Pileus deeply infundibuliform, $5-8 \times 0.1-0.3 \mathrm{~cm}$.; surface glabrous, rufous, very rugose when dry; margin thin, pellucid, entire, fertile, much deflexed in herbarium specimens; context fleshy, fragile when dry, white to pallid, I-2 mm. thick; tubes decurrent almost to the base of the long stipe, I mm. long, 4-5 to a mm., white to yellowish-brown, mouths angular, quite regular, edges thin, fragile, subentire to elongate, more or less collapsing on drying; stipe central, solid, somewhat curved, concolorous with the hymenium, pulverulent, $2.5-4 \mathrm{~cm}$. long, 4-6 mm. thick.

Occasional on dead logs in Cuba and Santo Domingo. $P$. craterellus is not distinct.

\section{Polyporus Acicula Berk. \& Curt.}

Pileus circular, convex, umbilicate, resembling a minute Omphalia in shape, $2 \times 0.5 \mathrm{~mm}$.; surface glabrous, minutely concentrically rugose when dry, yellowish-brown in the single type specimen; margin acute, involute; context membranous, white; tubes small, alveolar; stipe central, filiform, reddishbrown when dry, glabrous above, slightly villose at the base, $6 \mathrm{~mm}$. long, $0.25 \mathrm{~mm}$. thick.

Found once on dead wood in Cuba.

\section{Polyporus discoideus Berk. \& Curt.}

Pileus circular, plane, IO- $12 \mathrm{~cm}$. in diameter, $\mathrm{I}-2 \mathrm{~cm}$. thick at the center, tapering to a few millimeters near the periphery; surface smooth, glabrous, pale-ochraceous to umbrinous; margin darker, thin, straight, entire; context white to yellowish, very soft-corky, elastic, $0.5^{-1} \mathrm{~cm}$. thick; tubes pallid to dull-brown, mouths subangular, becoming sinuose, 2 to a mm., edges thin, elongate-dentate, collapsed when dry; stipe central, thick, even, hispid, concolorous above, darker and radicate below, $6 \mathrm{~cm}$. long, $\mathrm{I} \cdot 5^{-2} \mathrm{~cm}$. thick.

Found once on dead wood in Cuba.

\section{Polyporus obolus Ellis \& Macbr.}

Pileus circular, plane, $\mathrm{I}-2.5 \times 0.03-0.08 \mathrm{~cm}$.; surface minutely tomentose, radiate-rugose, isabelline, fulvous at the center; margin straight or repand, even, glabrous, entirely devoid of teeth or cilia; context $0.2-0.7 \mathrm{~mm}$. thick, tough, white, translucent, especially near the margin; tubes $0.1 \mathrm{~mm}$. long, 8 to a mm., adnate, white, cylindric, regular, edges thick, entire; spores 
ovoid, $4.5^{-5} \times 3.5^{-4} \mu$; stipe central, tough, elastic, slender, equal, chestnut-colored, glabrous, smooth, much compressed in drying, I-4 cm. long, I-2 mm. thick.

Occasional on dead sticks in moist situations in Nicaragua, British Honduras, Porto Rico, and Guadeloupe. Leucoporus dictyoporus Pat., described from Guadeloupe in 1908 , is not distinct.

\section{Polyporus aemulans Berk. \& Curt.}

Pileus thin, circular, umbilicate, $3.5-4 \times 0.05-0.1 \mathrm{~cm}$.; surface radiate-striate, ochraceous, dark-yellowish-brown when dry, glabrous at maturity; margin thin, straight, subcinereous, finely hispid, irregularly incised or lobed, sterile for I-2 mm.; context tough, membranous, pallid to pale-brown; tubes decurrent, yellowish, less than $\mathrm{I} \mathrm{mm}$. long, gradually disappearing near the margin, mouths alveolar, shallow, 2 to a mm., usually radially elongate, edges acute, becoming somewhat elongate; stipe central, equal, coffee-colored, pulverulent, expanding into the pileus, $2 \mathrm{~cm}$. long, 3-4 $\mathrm{mm}$. thick.

Found once on dead wood in Cuba.

\section{io. Polyporus arcularius (Batsch) Fries}

Pileus circular, convex, umbilicate, $\mathrm{I}-2.5 \times 0 . \mathrm{I}-0.2 \mathrm{~cm}$.; surface azonate, concentrically rugose when dry, fuscoussquamulose to fulvous and nearly glabrous; margin acute, ciliatedentate, straight, inflexed on drying; context white, membranous; tubes slightly decurrent, rather firm, white to brownish, I-2 $\mathrm{mm}$. long, mouths large, oblong-rhomboid, I-2 to a mm., edges thin, elongate, denticulate; spores cylindric, pointed at the ends, 2-guttulate, copious, $7-8 \times 1.5^{-2.5} \mu$; stipe central, slender, even, fuscous-gray to fulvous, subsquamulose to glabrous above, hispid-tomentose at the base, 2-4 cm. long, 2-3 mm. thick.

Occasional on dead wood in Mexico, Costa Rica, Jamaica, and Cuba; frequent in southern Florida.

\section{Polyporus VARIIPORUS Murrill}

Pileus orbicular, convex to depressed, $\mathrm{I}-2.5 \times 0 . \mathrm{I}-0.2 \mathrm{~cm}$.; surface glabrous, more or less radiately striate, somewhat concentrically rugose, straw-colored to isabelline, fulvous to chestnutcolored around the margin, which is thin, inflexed, undulate, finely ciliate, the cilia being fugacious; context $\mathrm{I}-1.5 \mathrm{~mm}$. thick, tough, white; tubes $0.5 \mathrm{~mm}$. in length, $2-4 \mathrm{~mm}$. in diameter, very irregular, much elongate radially near the stipe or in the 
marginal folds, not noticeably decurrent, yellowish, polygonal to lamelloid, edges firm, entire, becoming denticulate or fimbriate with age; spores ellipsoid, $7 \times 4 \mu$; stipe central, hollow, increasing above, lighter than the pileus, subglabrous, with silky luster, $2 \mathrm{~cm}$. long, 2-6 mm. thick, the buried base enlarged, tomentose, and frequently black.

Found once on buried sticks in Porto Rico.

\section{Polyporus Tricholoma Mont.}

Pileus circular, convex to infundibuliform, usually cespitose, I.5-4 $\times 0.05-0.2 \mathrm{~cm}$.; surface white or pallid to ochraceous or reddish-brown, azonate, fibrillose to glabrous; margin straight or inflexed, conspicuously ornamented with rigid hairs, or cilia, $2 \mathrm{~mm}$. long and of uncertain duration; context pallid, membranous, tough; tubes slightly decurrent, white to pallid, $0.5^{-1} \mathrm{~mm}$. long, mouths angular, 5 to a $\mathrm{mm}$., edges thin, dentate, elongate; spores ellipsoid, $6-7 \times 2-2.5 \mu$; stipe central, equal, glabrous, concolorous, very slender, $1.5^{-2.5} \mathrm{~cm}$. long, $\mathrm{I}-\mathbf{r} .5 \mathrm{~mm}$. thick.

Very common throughout on dead sticks and logs in woods.

\section{Polyporus Cowellit Murrill}

Pileus orbicular, convex to plane, umbilicate, $\mathbf{I}-2 \times 0.02-0.05$ $\mathrm{cm}$.; surface nearly glabrous, minutely concentrically rugose, straw-colored to isabelline, becoming darker and hygrophanous around the margin or in blotches or even over the entire surface, often faintly radiate-striate about the center with delicate white or brown lines; margin very thin, straight or inflexed, somewhat irregular in outline, finely denticulate, the teeth prolonged into short, fugacious cilia; context extremely thin, membranous, $0.1-0.2 \mathrm{~mm}$. thick, white or pallid, partially or entirely translucent; tubes $0.2-0.4 \mathrm{~mm}$. long, 3-6 to a mm., larger by confluence, adnate, pallid, polygonal, edges very thin, subentire, becoming fimbriate with age; spores ellipsoid, $5 \times 2.5 \mu$; stipe central, solid, very slender, equal, concolorous or slightly darker, pruinose to glabrous, smooth, longitudinally striate, $2-3 \mathrm{~cm}$. long, 0.3-I mm. thick, soft and milk-white at the center.

Found a few times on dead wood in Porto Rico and St. Kitts.

\section{Polyporus maculosus Murrill}

Pileus irregularly orbicular, deeply depressed, $3-3.5 \times 0.1-0.2$ $\mathrm{cm}$.; surface finely and densely tomentose, radiately striate, drab-colored, ornamented with several light-bay or chestnut spots mostly situated about midway between the center and the 
margin, which is very thin, striate, irregular and somewhat fissured but entirely devoid of teeth or cilia; context $0.5-1.5 \mathrm{~mm}$. thick, tough to corky, pallid; tubes $0.2-0.4 \mathrm{~mm}$. long, $6-7$ to a mm., decurrent, pallid or yellowish, subcylindric, edges entire; spores ovoid, $4 \times 3 \mu$, immature in the type specimens; stipe central, solid, woody, tapering upward, chestnut-black at the base, with blotches of light-yellow, uneven, subglabrous, $2 \mathrm{~cm}$. long, 2-4 mm. thick.

Found once on dead wood in Central America.

\section{I5. Polyporus WrightiI Murrill}

Pileus circular, convex-plane, umbilicate, $3-4 \times$ o.I $\mathrm{cm}$.; surface delicately radiate-striate, glabrous, yellowish-brown to isabelline; margin thin, acute, bay, irregularly undulate or lobed, slightly incurved, not ciliate; context membranous, pallid, corky, less than $0.5 \mathrm{~mm}$. thick; tubes decurrent, umbrinous when dry, 0.5-1 mm. long, 2-3 to a mm., mouths irregular, angular, very variable in size, edges thin, entire, slightly toothed with age; stipe short, central, solid, woody, equal or slightly enlarged at the base, pulverulent, black, 5-7 mm. long, 2-3 mm. thick.

Found once or twice on dead wood in Cuba.

\section{i6. Polyporus marasmioides (Pat.) Murrill}

Pileus circular, convex-plane, deeply umbilicate, sometimes entirely aborted, I $-5 \times 0 . I \mathrm{~cm}$.; surface glabrous, ochraceous, obscurely striate near the margin, marked with a shallow, circular, peripheral furrow; margin straight, yellowish, translucent, puberulent, denticulate, sterile; context pallid, corky; tubes large, shallow, radiating, angular, I $\mathrm{mm}$. or more in diameter, edges thick, hard, entire, becoming denticulate or lacerate; spores elongate-ovoid, guttulate, Io $\times 4 \mu$; stipe central, cylindric, brownish-black, white within, tenacious, woody, velvety-hispid, I-I $5 \mathrm{~cm}$. long.

Found a few times on decaying seeds or dead trunks in Guadeloupe and Martinique.

\section{I7. Polyporus elegans (Bull.) Fries}

Pileus flabelliform to subcircular, scarcely depressed behind, convex or nearly plane, $2-6 \times 3-10 \times 0.2-1 \mathrm{~cm}$.; surface distinctly radiate-striate, pruinose when young, becoming glabrous and pale-ochraceous at maturity; margin thin, at first inflexed, often becoming wavy or much lobed and folded with age, not 
ciliate; context white or pallid, corky, $\mathrm{I}-5 \mathrm{~mm}$. thick; tubes paleavellaneous, I-3 mm. long, cylindric, mouths angular to subcircular, entire, at first white, glistening, pale-umbrinous with age, 4-5 to a mm., edges thin, entire; spores oblong, 7-8 $\times 3$-3.5 $\mu$; stipe eccentric or lateral, rarely central, woody, smooth, pallid above, abruptly black and scutate below, $\mathrm{r}-4 \mathrm{~cm}$. long, 2-5 mm. thick.

Occasional on fallen dead branches at Jalapa, Mexico.

\section{I8. PolyPorus Subelegans Murrill}

Pileus flabelliform, convex, of ten attenuate at the base, $\mathrm{I}-2 \times$ I.5-2.5 $\times 0.1 \mathrm{~cm}$.; surface delicately radiate-striate, glabrous, isabelline to pale-umbrinous when dry; margin acute, entire, undulate to lobed, rarely inflexed; context pallid, corky, 0.5 $\mathrm{mm}$. thick; tubes pale-umbrinous, $\mathrm{I} \mathrm{mm}$. long, cylindric, mouths white when young, perfectly circular, exceedingly minute, Io to a mm., edges thick, entire; stipe short, lateral, solid, woody, scutate at the base, black, pruinose, rugose, 2-5 $\mathrm{mm}$. long, 2-3 mm. thick.

Frequent on dead sticks in Mexico, Nicaragua, and Jamaica.

\section{Polyporus diabolicus Berk.}

Pileus circular, umbilicate, convex, 3-10 $\times 0.2-0.4 \mathrm{~cm}$.; surface bright-bay to almost black, usually radiate-striate, pruinose to glabrous, sometimes proliferous; margin rather thin, entire, concolorous to crenate or lobed, at first straight, becoming slightly deflexed; context white or pallid, corky, I-3 mm. thick; tubes white to umbrinous, $\mathbf{I}-2 \mathrm{~mm}$. long, mouths minute, circular, 4-6 to a mm., edges thick, entire; stipe central, solid, woody, equal or slightly tapering upward, concolorous, glabrous, $3-8 \mathrm{~cm}$. long, 3-10 mm. thick.

Occasional on dead trunks in Mexico and Central America, as well as in South America.

\section{Polyporus Marbleae Murrill, sp. nov.}

Pileus very convex above and concave below, laterally attached, $6 \times 8 \times \mathrm{I}-2 \mathrm{~cm}$.; surface smooth, glabrous, shining, fuliginous, margin acute, concolorous, undulate, shortly inflexed on drying; context white, fragile, homogeneous, about $7 \mathrm{~mm}$. thick; hymenium pale-avellaneous when young; tubes equaling the thickness of the context, minute, whitish or yellowish, thinwalled, the edges becoming fimbriate-lacerate; stipe short, lateral, avellaneous or slightly darker, smooth, glabrous, marked 
above with the decurrent tubes, about $2 \mathrm{~cm}$. long and I-I.5 cm. thick.

Type collected on dead wood at Mount Morales near Utuado, Porto Rico, March 19, 1906, Elizabeth G. Britton \&o Delia W. Marble 1216 (herb. N. Y. Bot. Gard.). It is known only from this single collection, which, unfortunately, was attacked by insects while developing and the tubes are consequently abnormal.

\section{Polyporus Blanchetianus Berk. \& Mont.}

Pileus semicircular or reniform, plane or slightly convex, 2-5 $\mathrm{cm}$. broad, $0.5 \mathrm{~cm}$. thick; surface bay to black, very glabrous, often striate or slightly veined; margin acute, entire to wavy or lobed; context corky, pallid to brownish, 2-4 mm. thick; tubes very short, minute, circular or subangular, pallid to umbrinous, I-2 mm. long, 5-6 to a mm., edges thick, entire; spores oblong, inequilateral, $6-7 \times 2-3 \mu$; stipe lateral to eccentric, short, black, opaque, punctulate, 2-Io $\mathrm{mm}$. long, 2-3 mm. thick.

Frequent throughout on dead wood; also in South America.

\section{Polyporus scabellus (Pat.) Murrill}

Pileus semicircular or nearly reniform, plane, slightly elevated behind, 3-8 $\times$ 4-I0 $\times 0.1-0.5 \mathrm{~cm}$.; surface bright-chestnutcolored, finely radiate-striate or marked with rows of serrated, black squamules; margin straight, acute, entire; context white, becoming brownish on drying, membranous; tubes very short, not decurrent, mouths light-brown, more or less tinged with fuliginous near the stipe, round, very small, the dissepiments: thick; stipe eccentric, horizontal, cylindric, very short, black, minutely velvety, marked with punctate depressions, 3-4 $\mathrm{mm}$. long, 3-6 mm. thick, inserted upon a black, orbicular disk.

Occasional on dead hardwood trunks in Guadeloupe.

\section{Polyporus praeguttulatus Murrill}

Pileus subcircular in outline, convex above, nearly plane below, attached by a decurrent base, which causes the hymenophore to appear triangular in section, $4 \times 4.5 \times 0.5^{-2.5} \mathrm{~cm}$.; surface smooth, latericeous, with a coating of fine, grayish tomentum, which disappears in numerous subcircular spots; margin slightly upturned, abruptly acute, concentrically striate, entire, cremeous; context pallid, soft-corky, homogeneous, I cm. thick behind; tubes stramineous, 3-4 mm. long, mouths subcircular to angular, 
larger near the margin, most of them about 3 to a mm., glistening, stramineous, edges thin, entire; spores ovoid, $5-6 \times 2-3 \mu$; stipe obsolete.

Collected once in Troy and Tyre, Jamaica, on a log in woods.

\section{I6. ABORTIPORUS Murrill}

Hymenophore annual, tough, humus-loving; stipe normally central, often obsolete; context yellowish-white, duplex, spongy above, woody below, tubes thin-walled, mouths polygonal; spores smooth, hyaline.

Pileus circular, 6- $13 \mathrm{~cm}$. broad.

I. A. distortus.

Pileus spatulate to reniform, 2-3 cm. broad.

2. A. tropicalis.

\section{Abortiporus distortus (Schw.) Murrill}

Pileus normally thin, plane or depressed, circular and centrally stipitate when properly developed, but often aborted and very irregular, varying to entirely resupinate forms, $6-13 \mathrm{~cm}$. in diameter, $0.3^{-1} \mathrm{~cm}$. thick; surface conspicuously and compactly tomentose, anoderm, azonate, smooth, white to alutaceous; margin thin, undulate to lobed, concolorous; context soft and spongy above, hard and woody below, white or isabelline, 3-5 mm. thick; tubes annual, decurrent, white, I-5 mm. long, mouths irregular, variable, $2-3$ to a mm., edges thin, entire to dentate; spores subglobose, 5-7 $\mu$ long; stipe central, unequal, very variable, often obsolete, resembling the pileus in surface and context.

Found twice in Porto Rico, once in the perfect form and once in the aborted condition. It usually occurs about stumps and buried wood of broad-leaved trees.

\section{ABortiporus tropicalis Murrill}

Pileus distorted, spatulate to reniform, laterally stipitate, tough, flexible, becoming almost rigid when dry, $\mathrm{I}-2 \times 2.5-3 \times$ $0.2-0.4 \mathrm{~cm}$.; surface spongy, finely tomentose, uneven, plane or depressed, rosy-ochraceous, anoderm; margin white, becoming reddish-brown when bruised, undulate to lobed, sterile; context white, duplex, with a firmer layer next to the tubes; tubes short, white within, mouths minute, invisible to the unaided eye, somewhat glistening, white, edges firm, obtuse to subacute, entire; spores globose, smooth, thin-walled, hyaline, copious, 4.5-6 $\mu$; stipe ascending, expanding into the pileus, sometimes branched at the base, very irregular, cremeous, clothed like the pileus, tapering below, about $3 \mathrm{~cm}$. long and 5-10 mm. thick. 
Collected once in Hope Gardens, Jamaica, attached to the base of a small dead stump, and once at Jalapa, Mexico; also in South America.

\section{I7. GRIFOLA (Micheli) S. F. Gray}

Hymenophore large, annual, stipitate, compound, intricately branched or lobed, humus-loving or epixylous, rarely terrestrial, usually found at the base of a tree-trunk; surface smooth, pallid to gray or brown; context white, fleshy or fleshy-tough, rigid and fragile when dry; tubes large, irregular, thin-walled, becoming friable or laciniate with age; spores hyaline, smooth or rarely verrucose.

\section{Grifola lentifrondosa Murrill}

Pileus imbricate-multiplex, $15-20 \times 20-30 \mathrm{~cm}$.; pileoli mostly spatulate, closely appressed and more or less connate; surface isabelline with a rosy tint, subtomentose, becoming fuliginous with age, margin entire or undulate, sterile, inflexed on drying; context white, radiate-fibrous, separating into tough strings like that of bast-tissue; tubes pallid to light-brown, minute, thinwalled, 2-3 mm. long, mouths angular, edges thin, lacerate with age; spores globose, smooth, hyaline, copious, 3-4 $\mu$; stipe tubercular, woody, pallid, expanding into the pileoli, $8 \mathrm{~cm}$. long, $6 \mathrm{~cm}$. thick.

Collected once on the roots of an oak stump on an exposed railway embankment near Jalapa, Mexico.

\section{PYCNOPORUS P. Karst.}

Hymenophore annual, sometimes reviving, epixylous, sessile, dimidiate, simple or imbricate, rarely pseudo-stipitate; surface anoderm, slightly pelliculose at times, zonate or azonate, brightor dull-red; context red, soft-corky to punky; hymenium concolorous, tubes small, firm, thin-walled; spores smooth, hyaline.

\section{Pycnoporus sanguineus (L.) Murrill}

Pileus thin, coriaceous, sessile or spuriously stipitate, dimidiate, conchate or reniform, imbricate, laterally connate at times, 3-5 $\times$ 4-8 $\times 0.4-0.6 \mathrm{~cm}$.; surface zonate, finely tomentose to glabrous, bright-red, often variegated with yellowish-red zones, fading to pure-white in old specimens exposed to the sun; margin acute, finely tomentose, yellowish-red; context floccose, elastic, yellowish-red, I-3 mm. thick; tubes annual, very short, bright- 
reddish-miniatous, scarcely a $\mathrm{mm}$. long, mouths circular to angular, regular, minute, 3-5 to a mm., edges thin, firm, entire, concolorous with the interior; spores oblong, 3-4 $\times 1-2 \mu$.

Extremely common throughout on all forms of dead wood. A few specimens have been seen from Mexico and the Bahamas which greatly resemble $P$. cinnabarinus.

\section{FLAVIPORELLUS Murrill}

Hymenophore small, annual, epixylous, sessile or substipitate, flabelliform, yellow throughout; surface anoderm, margin thin; context very thin and friable; tubes small, thin-walled, fragile; spores smooth, hyaline or yellowish.

\section{Flaviporellus Splitgerberi (Mont.) Murrill}

Pileus thin, dimidiate to spatulate-cuneate, densely imbricate, gibbous behind, $2-4 \times 3-8 \times 0.1-0.2 \mathrm{~cm}$.; surface anoderm, fibrose-radiate, subzonate, smooth, flavous-luteous with reddishfulvous spots or zones, fulvous or umbrinous behind; margin very thin, subfibrous, inflexed or folded when dry, broadly sterile, golden-yellow; context radiate-fibrous, corky to woody, very hard next to the tubes, I $\mathrm{mm}$. thick, shining-flavous; tubes annual, melleous within, scarcely a $\mathrm{mm}$. long, mouths circular to angular, 4-6 to a mm., pale-luteous to melleous, edges very thin, becoming dentate-lacerate with age; spores ovoid or ellipsoid, subhyaline or pale-luteous, 4-5 $\times 3 \mu$.

Occasional on dead wood in Mexico, Panama, and Cuba; also in South America.

\section{LAETIPORUS Murrill}

Hymenophore annual, epixylous, fleshy, anoderm, cespitosemultiplex; context cheesy to fragile, light-colored; tubes thinwalled, fragile, bright-yellow, mouths irregularly polygonal; spores smooth, hyaline.

\section{LAETIPORUS SPECIOSUS (Batt.) Murrill}

Hymenophore cespitose-multiplex, 30-60 cm. broad; pileus cheesy, not becoming rigid, reniform, very broad, more or less stipitate, $5^{-1} 5 \times 7^{-20} \times 0.5^{-1} \mathrm{~cm}$.; surface finely tomentose to glabrous, rugose, anoderm, subzonate at times, varying from lemon-yellow to orange, fading out with age; margin thin, fertile, concolorous, subzonate, finely tomentose, undulate, rarely lobed; context cheesy, very fragile when dry, yellow when fresh, 
usually white in dried specimens, 3-7 mm. thick; tubes annual, 2-3 mm. long, sulfur-yellow within, mouths minute, angular, somewhat irregular, 3-4 to a $\mathrm{mm}$., edges very thin, lacerate, sulfur-yellow, the color fairly permanent in dried specimens; spores ovoid, smooth or finely papillate, $6-8 \times 3-5 \mu$.

Occasional in Cuba and Mexico on living trunks of various trees, causing heart-rot. This species is cosmopolitan, but evidently does not thrive under tropical conditions.

\section{PHAEOLOPSIS Murrill}

Hymenophore annual, epixylous, stipitate; surface azonate, anoderm, yellow or brown; margin acute; context yellow, fleshy to tough and fibrous, not friable; tubes yellow, regular, minute, thin-walled; spores smooth, hyaline; stipe eccentric or lateral, with substance and surface like that of the pileus.

\section{Phaeolopsis Verae-crucis (Berk.) Murrill}

Pileus fleshy to tough, becoming rigid, flabelliform, with stipe lateral or eccentric, depressed behind, 5-6 $\times 7-8 \times 0.2-0.3 \mathrm{~cm}$.; surface glabrous, radiate-striate, bright-fuscous, darker with age; margin acute, undulate to lobed, inflexed when dry; context dark-yellowish-orange in dried specimens, rhubarb-yellow when fresh, tough and fibrous, glistening, $\mathrm{I}-2 \mathrm{~mm}$. thick; tubes annual, about I mm. long, rhubarb-yellow when fresh, becoming fuscous, mouths minute, circular, regular, $6-7$ to a mm., edges thin, equal, entire; stipe attenuate below, rhubarb-yellow when fresh, rough, slightly tomentose, resembling the context within.

Collected once in Vera Cruz, Mexico, growing on the roots of trees, and once on dead wood in British Honduras.

\section{CERRENELLA Murrill}

Hymenophore thin, effused-reflexed, annual, epixylous; surface brown, zonate, anoderm, margin thin; context thin, coriaceous, brown; hymenium at first poroid, very soon becoming irpiciform, the teeth irregular and compressed; spores smooth, hyaline.

Pileus very thin, I mm. or less in thickness.

Hymenium ferruginous, unchanging.

Hymenium olivaceous, becoming cinereous.

Pileus $3-7 \mathrm{~mm}$. thick; hymenium chestnut-colored to almost black.
I. C. Ravenelii.

2. C. farinacea.

3. C. subcoriacea. 


\section{Cerrenella Ravenelit (Berk.) Murrill}

Pileus thin, coriaceous, flexible, effused-reflexed, the reflexed portion dimidiate, imbricate, connate, o-I $\times \mathrm{I}-3 \times 0.1 \mathrm{~cm}$.; surface tomentose to finely hirsute, finely concentrically striate, fulvous-chestnut to avellaneous; margin very thin, undulate to lobed, deflexed in dried specimens; context papery thin, concolorous; tubes short, irregular, less than $\mathrm{I} \mathrm{mm}$. in length, I-2 to a mm., teeth ferruginous, compressed, obtuse, somewhat seriate, irregular in size and shape, pulverulent at first; spores globose, smooth, hyaline, 6-7 $\mu$.

Occasional in southern Florida on decaying branches of oak and other deciduous trees.

\section{Cerrenella farinacea (Fries) Murrill}

Pileus very thin, soft, flexible, coriaceous, entirely resupinate or effused-reflexed, the reflexed portion dimidiate, imbricate, laterally connate, $0-\mathrm{I} \times \mathrm{I}-5 \times 0.1 \mathrm{~cm}$.; surface finely concentrically striate, tomentose, umbrinous-chestnut; margin very thin, undulate to lobed, sterile; context membranous, concolorous, papery-thin; tubes short, I mm. or less, irregular, 2-3 to a mm., edges thin, fimbriate to lacerate, dentate, separated at a very young stage forming an irpiciform hymenium, yellowishgreen to olive and finally cinereous and farinaceous; spores $6-7 \times 2.5 \mu$.

Frequent throughout on decaying branches of hardwood trees.

\section{Cerrenella subcoriacea Murrill}

Pileus coriaceous, rather flexible, effused-reflexed, the reflexed portion dimidiate, imbricate, often laterally connate, $\mathrm{I}-2 \times 2-5$ $\times 0.3-0.7 \mathrm{~cm}$.; surface somewhat zonate, finely tomentose to glabrous, dark-chestnut-colored; margin entire to lobed, bay, tomentose, very thin; context very thin, less than I $\mathrm{mm}$., chestnut-colored, soft, fibrous; tubes often reviving, $2-4 \mathrm{~cm}$. long, umbrinous-chestnut and powdery within, hymenium irregular, daedaleoid to iripiciform, mouths $\mathrm{I}-2$ to a mm., edges thin, firm, glistening, chestnut-colored to very dark fuliginous, soon splitting into flat, more or less pointed teeth.

Found once on dead wood in Nicaragua.

\section{CORIOLOPSIS Murrill}

Hymenophore thin, flexible or rigid, annual, epixylous, sessile, dimidiate, often largely resupinate; surface light-brown to bay- 
black, zonate, anoderm, rarely encrusted with age, hairy; margin thin; context thin, coriaceous to woody, isabelline to purplishumbrinous, rarely almost white; hymenium concolorous; tubes small, regular, thin-walled, entire; spores smooth, hyaline.

Pileus isabelline to fulvous.

Pores inconspicuous; pileus papery-thin, very soft and flexible.

I. C. crocata.

Pores conspicuous.

Hymenium isabelline; pileu usually thick and firm.

Hymenium whitish or grayish, at least until matur-

ity; pileus thin, 1-3 mm., flexible, often narrowly reflexed or entirely resupinate.

Surface and hymenium whitish to isabelline.

Surface isabelline to fulvous or whitish-cinereous; hymenium murinous to umbrinous.

Tubes I-2 mm. long, 3-4 to a mm.

Tubes 3-5 $\mathrm{mm}$. long, 2 to a $\mathrm{mm}$.

Pileus wholly or partly darker than fulvous, usually baybrown or blackish.

Context isabelline to ochraceous-ferruginous.

Surface finely tomentose to scabrous.

Surface densely clothed with long fibers.

Fibers persistent; cystidia absent.

Fibers wearing away with age; cystidia conspicuous.

Context fulvous.

Pileus less than $2 \mathrm{~cm}$. broad.

Pileus $4-6 \mathrm{~cm}$. broad.

2. C. occidentalis.

3. C. rigida.

4. C. fulvocinerea.

5. C. crocatiformis.

Context umbrinous-chestnut.

Pileus effused, narrowly reflexed.

Tubes large, 2 to a mm., cinereous.

Tubes small, 7-8 to a mm., purplish-umbrinous.

Pileus not effused, or, if so, broadly reflexed.

Surface entirely pure-black, or slightly fuliginous

in narrow zones; hymenium grayish-white.

Surface fulvous-umbrinous with bay-black zones, or uniformly dark-bay.

Pileus flexible, tomentose to aculeate.

Pileus rigid, tomentose to glabrous; tubes of ten stratified.

II. C. vittata.

12. C. vibratilis.

7. C. cirrifer.

8. C. sarcitiformis.

9. C. fumosa.

10. C. caperatiformis.

13. C. nigrocinerea.

14. C. caperata.

15. C. subglabrescens.

\section{Coriolopsis crocata (Fries) Murrill}

Pileus coriaceous, membranous, flaccid, flexible, elastic, effusedreflexed, sometimes covering the entire under surface of logs, the reflexed portion dimidiate to reniform, applanate, conchate, $0-5$ $\mathrm{cm}$. long, 4 to many $\mathrm{cm}$. broad, not exceeding 2 or $3 \mathrm{~mm}$. in thickness; surface densely concentrically striate, somewhat zonate, 
villose-tomentose, grayish-isabelline or pale-fulvous; margin very thin, pallid, broadly sterile, entire to slightly undulate; context thin, membranous, isabelline, spongy, $1-2 \mathrm{~mm}$. thick; tubes very short and shallow, less than a $\mathrm{mm}$. in length, mouths punctiform, regular, circular, $4-5$ to a mm., edges thick, entire, white to isabelline or pale-fulvous.

Frequent throughout on dead hardwood logs.

\section{Coriolopsis occidentalis (Klotzsch) Murrill}

Pileus corky or leathery, dimidiate to reniform, applanate, 3-6 $\times$ 5-10 $\times 0.3^{-1} \mathrm{~cm}$.; surface concentrically sulcate, zonate, tomentose, grayish-ochraceous to grayish-isabelline, somewhat fading with age; margin thin, velvety, entire; context ochroleucous, subshining, zonate, corky, I-6 mm. thick; tubes rather short, 2-4 mm. long, isabelline within, mouths circular, slightly irregular at times, $2-4$ to a $\mathrm{mm}$., edges rather thick, becoming thin, entire, firm, milk-white when young and fresh, isabelline to ochraceous-fulvous at maturity; spores allantoid, 5-7 $\times 2-3 \mu$.

Extremely common on various kinds of dead wood throughout.

\section{CoRIOlopsis Rigida (Berk. \& Mont.) Murrill}

Pileus thin, coriaceous, flexible to rigid, effused-reflexed, imbricate, laterally connate, the reflexed portion flabelliform, applanate or conchate, $0.5-3 \times 3-6 \times 0.1-0.2 \mathrm{~cm}$.; surface spuriously zoned, sometimes zonate behind, concentrically furrowed at times, hirsute to hispid, nearly white to isabelline; margin very thin, pallid, undulate to lobed; context pallid to isabelline, membranous; tubes very short, grayish-isabelline within, mouths fairly regular, circular to slightly angular, 3-4 to a $\mathrm{mm}$., edges white to grayish-white and finally isabelline, rather thick at first, becoming thin, entire and slightly uneven.

Common on dead wood throughout.

\section{Coriolopsis fulvocinerea Murrill}

Pileus coriaceous, flexible to rigid, dimidiate, imbricate, laterally connate, often decurrent, usually conchate, $2-4 \times 3^{-8}$ $\times 0.2-0.4 \mathrm{~cm}$.; surface spuriously zonate, concentrically striate or sulcate, uneven, of ten plicate, isabelline to fulvous or cinereous, tomentose, scabrous behind; margin very thin, undulate to lobed, tomentose, isabelline to fulvous; context membranous, fibrous, pale-umbrinous, becoming darker with age, about I-2 $\mathrm{mm}$. thick; tubes short, I-2 $\mathrm{mm}$. long, cinereous to avellaneous within, mouths minute, regular, circular to angular, $3-4$ to a 
mm., elongate and irregular in larger specimens at times, murinous to umbrinous, edges thin, firm, entire.

Frequent on dead wood in Cuba, Jamaica, Santo Domingo, St. Croix, and Barbados.

\section{CORIOLOPSIS CROCATIFORMIS Murrill}

Pileus coriaceous, flaccid, flexible, elastic, effused-reflexed, subimbricate, more or less laterally connate, the reflexed portion dimidiate or laterally elongate, conchate, $\mathbf{I}^{-2}-2 \times 3^{-7} \times 0.2-0.5$ $\mathrm{cm}$.; surface very irregular and uneven, tomentose to spongy, somewhat sulcate, fulvous; context very thin, punky, fulvous; tubes 3-5 $\mathrm{mm}$. long, grayish-fulvous within; mouths large, angular, somewhat irregular, 2 to a mm., glistening, fulvousumbrinous, edges thin, flexible, entire or slightly toothed; spores subglobose, smooth, $3.5 \mu$.

Collected once on dead wood near Oaxaca, Mexico.

\section{CORIOLOPSIS TAYLORI Murrill}

Pileus corky, rigid, subimbricate, dimidiate, conchate, 3-5 $\times$ 4-7 $\times 0.5^{-1.5} \mathrm{~cm}$.; surface zonate, roughly hispid to scabrous, aculeate behind, somewhat sulcate, finely radiate-striate, subshining and chestnut-colored in zones near the margin and opaque and isabelline-fulvous behind, or entirely multizonate and subshining; margin thin, pallid, tomentose, entire or undulate; context isabelline, distinctly zonate, almost woody, $0.3^{-1} \mathrm{~cm}$. thick; tubes slender, 2-4 mm. long, circular, umbrinous within, mouths circular, regular, minute, $5-7$ to a mm., grayish-umbrinous to fuliginous, edges entire, becoming rather thin, but firm and even.

Frequent on dead logs in Panama, Honduras, Cuba, Porto Rico, St. John, and Jamaica.

\section{CorIOLOPSIS CIRRIFER (Berk. \& Curt.) Murrill}

Pileus dimidiate, sessile, subimbricate at times, usually conchate, $4-8 \times 7-10 \times \mathrm{r}-2 \mathrm{~cm}$.; surface zonate, at least when young, isabelline to fulvous or bay, becoming nearly fuliginous when old, conspicuously and very densely covered with long, branched hairs which are rather soft and yielding to the touch and do not disappear with age; margin entire, isabelline, rather thick and of ten obtuse; context isabelline, corky, 5-8 mm. thick; tubes slender, pale-avellaneous within, equaling the thickness of the context, mouths minute, circular, 4-5 to a mm., the edges thick, entire, pallid to umbrinous. 
Frequent on dead logs and stumps in Cuba, Porto Rico, and Central America. This species approaches Pogonomyces very closely, forming a connecting link between this genus and Coriolopsis.

\section{CORIOLOPSIS SARCITIFORMIS Murrill}

- Pileus thin, imbricate, dimidiate or flabelliform, applanate, usually several times lobed, narrowly attached, sessile, of light weight, 4-6 $\times$ 5-8 $\times 0.5-0.7 \mathrm{~cm}$.; surface slightly zonate at times, uneven, conspicuously adorned with dense, fulvous fibers arranged in a radiating manner and appearing as though originating from the tearing of the cuticle, these fibers gradually wearing away with age, leaving the surface still more uneven and from dark-fulvous to chestnut in color; margin usually rather deeply and many times lobed, thin, yellow below when young; context punky, ochraceous-ferruginous, varying in thickness from $I$ to 3 $\mathrm{mm}$.; tubes reaching $4 \mathrm{~mm}$. in length, pale-avellaneous within, corky; mouths minute, circular, $3-5$ to a mm., flavomelleous when young, becoming avellaneous or umbrinous and finally chestnut with age, glistening, edges uneven, rather thick at first, thin and lacerate with age; cystidia fulvous, ventricose at the base, tapering to a point, rather abundant, $20-40 \times 5^{-7} \mu$.

Collected three or four times in abundance on fallen dead trunks in a moist virgin forest in the Tepeite Valley, near Cuernavaca, Mexico. This interesting species has the habit of Hapalopilus gilvus and a surface covering similar to that of Pyropolyporus sarcitus. It is strictly annual and of light weight. No very near relative is known.

\section{CoRIOLOPsis fumosa Murrill}

Pileus small, thin, slightly flexible, somewhat bell-shaped, attached by the vertex to the under side of a dead branch, laterally connate, $0.7 \times 1.3 \times 0.1-0.2 \mathrm{~cm}$.; surface tomentose, narrowly concentrically zonate-sulcate, fulvous to bay, margin thin, undulate, fumosous beneath on drying; context fulvous, soft and spongy above, with a rather firm, fibrous layer next to the tubes; hymenium dark-fumosous-umbrinous; tubes less than I $\mathrm{mm}$. long, murinous within, firm, mouths regular, angular, very minute, 8-10 to a $\mathrm{mm}$., edges very thin, entire.

Collected once on dead wood in a moist virgin forest near Jalapa, Mexico. 


\section{IO. CORIOLOPSIS CAPERATIFORMIS Murrill}

Pileus slightly flexible to rigid, dimidiate, broadly attached, decurrent, applanate or slightly convex, 2.5-3.5 $\times 4-6 \times 0.2-0.7$ $\mathrm{cm}$.; surface shaggy with coarse, fulvous-bay hairs $5 \mathrm{~mm}$. in length, which gradually wear away with age and allow the fulvous or bay, zonate-sulcate surface to appear; margin undulate or lobed, thin, hairy to finely tomentose; context thin, firm, almost woody, fulvous; tubes $1.5^{-2.5} \mathrm{~mm}$. long, avellaneous-umbrinous within, mouths circular or irregular, not angular, 3-5 to a mm., edges rather thick, firm, entire, avellaneous to umbrinous.

Collected three times on dead wood in a moist virgin forest near Motzorongo, Mexico.

\section{I . CoRiolopsis vittata (Ellis \& Macbr.) Murrill}

Pileus very thin, flexible, coriaceous, laterally connate, effusedreflexed, $2-3 \times 6-10 \mathrm{~cm}$., the reflexed portion $0.5-1 \mathrm{~cm}$. long, 2 to many $\mathrm{cm}$. broad, scarcely a $\mathrm{mm}$. thick; surface tomentose, zonate, fulvous to bay-black, the zones nearly glabrous; margin very thin, sterile, isabelline, undulate to lobed, deflexed at times; context extremely thin, less than half a mm., tawny-bay, membranous; tubes short, scarcely a $\mathrm{mm}$. long, whitish-cinereous within, mouths large, regular, subhexagonal, 2 to a mm., edges thin, firm, subentire to fimbriate or slightly lacerate, cinereous or grayish to umbrinous.

Occasional on fallen dead deciduous branches in Mexico, Nicaragua, and Jamaica.

\section{I2. Coriolopsis vibratilis (Berk. \& Curt.) Murrill}

Pileus resupinate to shortly reflexed, thin, corky, I-3 mm. thick; surface finely tomentose, gilvous-umbrinous, azonate, anoderm; margin sterile, rather thick, entire or undulate; context membranous, I $\mathrm{mm}$. in diameter, soft-corky, purplishumbrinous; tubes short, I-2 mm., avellaneous within, mouths minute, regular, hexagonal, glistening, stuffed when young, 7-8 to a $\mathrm{mm}$., edges very thin, entire or slightly toothed, purplishumbrinous.

Found once on dead wood in Cuba.

\section{I3. Coriolopsis Nigrocinerea Murrill}

Pileus attached by a scutate disk or small tubercle, dimidiate to circular, subimbricate, thin, coriaceous, $2-3 \times 3-5 \times 0.2-0.5$ $\mathrm{cm}$.; surface hispid-tomentose, zonate, concentrically striate, 
fuliginous to jet-black; margin pallid, thin, entire to undulate, velvety; context membranous, fibrous, umbrinous to fuliginous, I-2 mm. thick; tubes short, I-2 mm. long, chalk-white within, mouths circular to angular, becoming more or less irregular and uneven, about 3 to a mm., edges avellaneous to cinereous, firm, subentire, rather thin.

Found once on an old pole on the summit of El Yunque, Cuba.

\section{I4. Coriolopsis caperata (Berk.) Murrill}

Pileus thin, coriaceous, flexible, dimidiate to flabelliform, narrowly attached, of ten laterally connate and subimbricate, sometimes decurrent, $4-7 \times 5^{-8} \times 0.2-0.3 \mathrm{~cm}$.; surface conspicuously tomentose, becoming aculeate, zonate, especially with age, uneven, isabelline to umbrinous, with bay or blackish zones; margin thin, undulate to lobed, sterile, almost white; context thin, punky above, corky below, umbrinous-chestnut, 2-4 mm. thick; tubes short, avellaneous within, $\mathrm{I}-2.5 \mathrm{~mm}$. long, mouths circular to subangular, regular, even, 3-4 to a mm., edges rather thick, firm, entire, pale-isabelline or grayish-avellaneous to pale-umbrinous; spores 8-10 $\times 3-4 \mu$.

Common on dead wood throughout.

\section{I5. Coriolopsis SUbGLABRESCENS Murrill}

Pileus slightly flexible to rigid, sometimes reviving for several seasons and presenting the appearance of a very thin Pyropolyporus, dimidiate to reniform, narrowly attached, decurrent, usually applanate, $4^{-6} \times 7^{-12} \times 0.4-1 \mathrm{~cm}$.; surface tomentose, sulcate, umbrinous to bay or blackish, becoming glabrous and bay-black; margin pallid, entire, acute; context thin, corky, purplish-umbrinous, 2-3 $\mathrm{mm}$. thick, becoming indurate and blackish-encrusted above with age; tubes grayish-umbrinous, of ten stratose, especially behind, $2-4 \mathrm{~mm}$. long, mouths circular, minute, regular, 5-6 to a mm., edges thick, entire, firm, avellaneous-isabelline to pale-umbrinous.

Frequent on dead wood in Cuba, Porto Rico, and Jamaica.

\section{FUNALIA Pat.}

Hymenophore annual, epixylous, sessile, dimidiate, of ten semiresupinate; surface anoderm, hairy to aculeate; context lightbrown, more or less duplex, spongy above, coriaceous to woody below; tubes usually large, thin-walled, more or less lacerate; spores smooth, hyaline. 
Context very thin, I-2 mm.

Surface villose, with simple hairs; hymenium pale-rose-tinted when fresh.

I. F. versatilis.

Surface strigose, with branched hairs; hymenium pallid to brown.

2. F. cladotricha.

Context usually quite thick, $3-15 \mathrm{~mm}$.

Surface hispid.

Surface aculeate.

3. F. hispidula.

4. F. aculeifer.

\section{Funalia versatilis (Berk.) Murrill}

Pileus effused-reflexed, imbricate, laterally connate, coriaceous, the reflexed portion $2-3 \times 3-6 \times 0.3-0.8 \mathrm{~cm}$.; surface villose, spuriously and opaquely zoned, grayish-white, becoming umbrinous to ferruginous behind with age; margin thin, acute, concolorous, undulate to lobed; context pallid, very thin, membranous, fibrous, scarcely a mm. thick; tubes slender, 5-7 mm. long, isabelline within, mouths very variable in size and shape, $0.5^{-2} \mathrm{~mm}$. broad, circular to angular or slightly daedaleoid, edges thin, entire to toothed, grayish-isabelline, pale-rose-tinted when fresh; spores oblong-allantoid, $6-8 \times 2-4 \mu$.

Common throughout on dead deciduous and coniferous wood. Funalia villosa (Sw.) Murrill is not distinct. Boletus villosus Sw. is Coriolus pinsitus (Fries) Pat., but Swartz' name cannot be used because $B$. villosus Huds. has priority.

\section{Funalia Cladotricha (Berk. \& Curt.) Murrill}

Pileus dimidiate, sometimes effused-reflexed, $2-5 \times 3^{-8} \times$ $0.5-0.8 \mathrm{~cm}$.; surface brown, conspicuously covered with branched, strigose hairs, which are somewhat concentrically arranged in zones, especially near the margin, which is thin, concolorous, undulate; context thin, soft-corky to spongy, brown, I-2 mm. thick; tubes long, uneven, irregular and variable in size, grayishbrown within, 3-7 $\mathrm{mm}$. long, mouths angular to daedaleoid, averaging I $\mathrm{mm}$. in breadth, edges thin, uneven to laceratetoothed, brown or grayish-umbrinous.

Found once on dead wood in Cuba.

\section{Funalia hispidula (Berk. \& Curt.) Murrill}

Pileus small, undulate, dimidiate, $\mathrm{I}-2 \times 2-4.5 \times 0.5^{-1} \mathrm{~cm}$.; surface ferruginous-umbrinous, hispid, azonate, anoderm; margin thick, at least when young, subtomentose, slightly paler, sterile; context zonate, fibrose-corky, shining-isabelline, 3-7 $\mathrm{mm}$. thick; tubes concolorous with the context, short, mouths circular and 
widely separated by thick dissepiments when young, $0.5^{-1} \mathrm{~mm}$. broad, pale-ferruginous, becoming darker with age.

Found several times on dead wood in Cuba.

\section{Funalia aculeifer (Berk. \& Curt.) Murrill}

Pileus irregularly effused to imbricate and dimidiate, triangular in section, $0.5^{-1} \times \mathrm{I}_{-2} \times 0.5^{-1.5} \mathrm{~cm}$.; surface isabelline, very uneven, broken up into projections, which vary in size and are decorated with rigid cylindric or flattened latericeous aculeae; margin thin, pallid, of ten indefinite or very irregular; context white, spongy, $0.5-1 \mathrm{~mm}$. thick, penetrated by the aculeae; tubes very irregular in size and shape, $2-3 \mathrm{~mm}$. long, white within, mouths polygonal to radially elongate, averaging about I $\mathrm{mm}$. in width, edges thin, uneven to toothed or lacerate.

Collected a few times on dead wood in Cuba and once at Jalapa, Mexico; also in South America.

\section{TRICHAPTUM Murrill}

Hymenophore annual, epixylous, sessile, dimidiate; context brown, firm and leathery below, very loosely fibrous and darker above; tubes short, thin-walled, mouths polygonal, at times becoming labyrinthiform; spores smooth, hyaline.

\section{Trichaptum trichomallum (Berk. \& Mont.) Murrill}

Pileus effused-reflexed, laterally connate, sometimes covering the entire under surface of dead logs, the reflexed portion dimidiate, conchate, $\mathrm{I}-10 \mathrm{~cm}$. long, 5 to many $\mathrm{cm}$. broad, 5-10 $\mathrm{mm}$. thick, thicker by overlapping; surface fuliginous to almost black, sometimes zonate, very conspicuously ornamented with long intricately-branched hairs; margin thin, sterile, concolorous, somewhat inflexed on drying; context light-brown, membranous below, very loosely fibrous and darker above, the entire plant, with the exception of the tubes and the very thin layer to which they are attached, being composed of the loose branched fibers mentioned above; tubes short, $\mathrm{I}-3 \mathrm{~mm}$. long, avellaneous within, mouths angular, of ten irregular and sometimes daedaleoid, about $0.5 \mathrm{~mm}$. broad, edges thin, entire, avellaneous, soon becoming lacerate or irpiciform.

Frequent throughout on dead logs. A very striking species.

\section{HAPALOPILUS P. Karst.}

Hymenophore annual, rarely perennial, epixylous, sessile, dimidiate, simple or imbricate; surface anoderm, rarely pellicu- 
lose, zonate or azonate, usually brown and glabrous; context brown, leathery or corky, tough or rarely friable when dry; hymenium usually differently colored, tubes small, thin-walled; spores small, usually ovoid, hyaline.

Context rigid; pileus azonate or with few and indefinite markings. r. H. gilvus.

Context flexible when hymenophore is expanded; pileus plainly and definitely multizonate.

2. H. licnoides.

\section{Hapalopilus Gilvus (Schw.) Murrill}

Pileus corky, dimidiate, imbricate, applanate or conchate, 3-6 $\times$ 5-ro $\times 0.5-1.5 \mathrm{~cm}$.; surface finely tomentose to glabrous, azonate, isabelline to fulvous, often marked with indistinct purplish-fuscous bands, rugulose to uneven; margin thin, ferruginous, entire to undulate, abruptly sterile; context ferruginous, fibrous-spongy to corky, zonate, 3-7 mm. thick; tubes short, slender, avellaneous to grayish-umbrinous within, 3-5 $\mathrm{mm}$. long, often found stratified, especially in the tropics, mouths small, regular, circular to angular, 6-8 to a mm., edges at first thick, pale-ferruginous, becoming thin, entire, glistening, olivaceousfuscous to purplish-fuscous; spores elongate-ellipsoid, 4-6 $\times 2-4 \mu$; cystidia chestnut-colored, ovate-subulate, $15-20 \times$ 4-5 $\mu$.

Very common throughout on dead wood of broad-leaved trees.

\section{Hapalopilus licnoides (Mont.) Murrill}

Pileus thin, coriaceous, flexible, imbricate, dimidiate, of ten narrowly attached, applanate or conchate, 3-6 $\times 4-8 \times 0.2-0.5$. $\mathrm{cm}$.; surface multizonate, concentrically striate, finely tomentose to partially glabrous, rather smooth, subshining, fulvous with bay zones; margin very thin, entire, ferruginous; context thin, ferruginous to fulvous, fibrous-spongy, I mm. thick; tubes short, I-2 mm., fulvous, glaucous near the mouths, which are very minute, regular, circular, $7-9$ to a $\mathrm{mm}$., edges thick, entire, pale-ferruginous to purplish-ferruginous; spores ellipsoid or subglobose, $3-4 \times 2-3 \mu$; cystidia subulate, chestnut-colored, 15-25 $\times 6 \mu$.

Very common throughout on various kinds of dead wood.

\section{FAVOLUS Beauv.}

Hymenophore small, annual, epixylous, sessile, dimidiate or reniform; surface multizonate, margin thin; context thin, leathery, isabelline or fulvous; tubes alveolar; spores smooth, hyaline. 
Tubes visible to the unaided eye.

Surface marked with white and light-brown zones.

I. F. tenuis.

Surface variegated with dark-brown and purple zones.

2. F. variegatus.

Tubes invisible to the unaided eye.

3. F. tenuiformis.

\section{Favolus tenuis (Hook.) Murrill}

Pileus coriaceous, reniform, conchate or applanate, narrowly attached behind, $3^{-6} \times 5^{-1}$ I $\times 0.1-0.3 \mathrm{~cm}$.; surface glabrous, polished, pale-wood-colored to avellaneous-umbrinous, repeatedly narrowly zonate; margin very thin, usually entire or slightly undulate; context membranous, pale-isabelline, $0.5^{-1} \mathrm{~mm}$. thick; tubes very short, I-2 $\mathrm{mm}$. long, avellaneous within, mouths alveolar, circular to hexagonal, exceedingly variable in size in different collections, averaging $0.5^{-I} \mathrm{~mm}$. in diameter, edges rather thick, firm, entire.

Common throughout on dead hardwood trunks and branches.

\section{Favolus variegatus (Berk.) Murrill}

Pileus dimidiate to reniform, thin, coriaceous, $4^{-6} \times 8-12 \times$ 0.I-0.4 cm.; surface radiate-rugose, multizonate, velvety, becoming glabrous, chestnut-colored, variegated with chocolatebrown or bay-brown tints; margin very thin, slightly paler, blackening when bruised, entire to lobed; context very thin, I $\mathrm{mm}$. or less thick, fibrous, isabelline to umbrinous; tubes very short, pale-umbrinous within, scarcely a $\mathrm{mm}$. long, alveolar, subcircular to hexagonal, $\mathrm{I}-2$ to a $\mathrm{mm}$., edges rather thick, firm, even, pallid to pale-umbrinous.

Occasional throughout on dead wood. Abundant in some parts of southern Florida, where the hymenophores often reach a foot in diameter and are locally known as "spirit-cups."

\section{Favolus tenuiformis Murrill}

Pileus very thin, coriaceous, flexible, fan-shaped, applanate, narrowly attached, $2 \times 2.5 \times 0.1-0.2 \mathrm{~cm}$.; surface glabrous, shining, zonate, slightly radiate-rugose, umbrinous to palechestnut, margin very thin, entire, concolorous; context lightfulvous, membranous, as thin as paper; tubes less than I mm. long, avellaneous-umbrinous within, mouths circular, irregular, 6 to a mm., edges fuliginous, at first thick, becoming rather thin, firm, entire.

Collected once on dead wood in a dense jungle at Tecoman, near Colima, Mexico. This species resembles Favolus tenuis, but has much smaller tubes, the mouths being invisible to the unaided eye. 


\section{FLAVIPORUS Murrill}

Hymenium annual, of ten reviving, epixylous, sessile, dimidiate, imbricate; surface encrusted, glabrous; context thick, woody, brown; hymenium yellow to orange; tubes thin-walled, minute, regular; spores smooth, hyaline.

Hymenium pale-lemon-yellow.

I. F. rufoflavus.

Hymenium deep-orange-colored.

2. F. crocitinctus.

\section{Flaviporus rufoflavus (Berk. \& Curt.) Murrill}

Pileus dimidiate, $I \times 2-3 \times 0 . I-0.4 \mathrm{~cm}$.; surface rufous, thinly encrusted, finely pubescent to glabrous, polished, zonate; margin thin, undulate; context thin, pallid, less than I mm. thick; tubes slender, I-2 mm. long, yellowish, mouths very minute, angular, citrinous, 7-Io to a mm., edges thin, entire.

Occasional on decaying wood throughout most of tropical America; also in hothouses and mines in Europe.

\section{Flaviporus CROCitinctus (Berk. \& Curt.) Murrill}

Pileus hard, rigid, dimidiate, laterally connate, subimbricate, $3 \times 5 \times 0.3-0.5 \mathrm{~cm}$.; surface brown to black, thinly encrusted, sulcate, zonate, rugose, finely tomentose to glabrous, subshining; margin thin, finely tomentose, yellowish, lobed in type specimens; context thin, fibrous, flavous to luteous, scarcely I mm. thick; tubes slender, 2-4 mm. long, yellow to ferruginous, mouths circular to angular, stuffed when young, $4-5$ to a mm., edges thin, entire, flavous to luteous.

Found once on decaying wood in Cuba.

\section{POGONOMYCES Murrill}

Hymenophore annual, epixylous, dimidiate-sessile to flabelliform, thickly covered with rigid hairs; context dark-brown, punky to corky; tubes short, thick-walled, light-brown, mouths small, circular; spores smooth, hyaline.

\section{Pogonomyces hydnoides (Sw.) Murrill}

Pileus dimidiate, sessile, of ten imbricate, conchate, $3^{-5} \times 5^{-10}$ $\times 0.5^{-1} \mathrm{~cm}$.; surface zonate, tawny-bay to nearly black, conspicuously and thickly covered with rigid, branched fibers, which of ten more or less disappear with age; margin entire or undulate, pallid, acute, sterile below; context fulvous at maturity, zonate, punky to corky, 3-5 $\mathrm{mm}$. thick; tubes rather long, grayishumbrinous within, equaling the thickness of the context, mouths 
small, circular to somewhat angular, 3-4 to a mm., edges thick, entire, pallid to umbrinous; spores oblong, 8-10 $\times 3-4 \mu$.

Extremely common throughout on various forms of dead wood.

\section{NIGROPORUS Murrill}

Hymenium annual, epixylous, dimidiate-sessile to flabelliform, glabrous; context dark-brown, firm, homogeneous; tubes short, slender, thin-walled, black; spores smooth, hyaline.

\section{Nigroporus vinosus (Berk.) Murrill}

Pileus thin, dimidiate to reniform, imbricate, narrowly attached by a scutate disk or laterally connate and broadly decurrent, I-3 $\times 3-6 \times 0.1-0.3 \mathrm{~cm}$.; surface zonate, finely velvety to glabrous, obscurely vinous-brown; margin yellowish when young, thin, undulate, often inflexed on drying; context rigid, tough, chestnut-colored, I-2 mm. thick; tubes minute, short, scarcely a mm. in length, smoky-black, mouths regular, angular, 7-9 to a mm., edges thin, entire, vinous-brown, pruinose when young, blackish with age; spores allantoid, $3-4 \times \mathrm{I}-\mathrm{I} .5 \mu$.

Frequent on dead wood in Cuba, Santo Domingo, Guadeloupe, Martinique, and British Honduras.

\section{CYCLOPORELLUS Murrill}

Hymenophore annual, tough, epixylous, sessile, anoderm, zonate; context thin, fibrous, brown; tubes short, thin-walled, mouths polygonal, becoming concentrically elongate in some species by the splitting of the radial walls; spores ovoid, smooth, pale-ferruginous.

\section{Cycloporellus iodinus (Mont.) Murrill}

Pileus conchate, thin, umbonate-sessile, simple or imbricate, of ten radially-plicate, $2-4 \times 3-5 \times 0.1-0.2 \mathrm{~cm}$.; surface densely concentrically zonate, velvety, tawny-chestnut; margin thin, entire, ferruginous, deflexed in drying; context leathery, slightly flexible, fulvous; hymenium fuliginous, tubes I $\mathrm{mm}$. or less in length, 3-7 to a mm., isabelline within, polygonal and regular, often becoming concentrically confluent, edges thin, entire, often splitting with age; spores pale-ferruginous, $5 \times 3 \mu$; cystidia dark-brown, rather scanty, averaging $20 \times 5 \mu$.

Frequent throughout on dead wood of frondose trees. 


\section{INONOTUS P. Karst.}

Hymenophore annual, epixylous, sessile, dimidiate, simple or somewhat imbricate, variable in size; surface usually anoderm, brown, hairy or glabrous; context brown, thin and fibrous to spongy or corky; hymenium concolorous, usually covered with whitish powder in youth, tubes small, thin-walled; spores smooth, light- to dark-brown.

Surface conspicuously hairy.

Pileus very thin, $1-2 \mathrm{~mm}$.

I. 1 . pertenuis.

Pileus quite thick, 7-20 $\mathrm{mm}$.

2. I. fulvomelleus.

Surface not conspicuously hairy.

Spores deep-brown in color.

Pileus $3 \mathrm{~cm}$. or less broad; spores ferruginous.

Pileus 5-I2 cm. broad; spores fulvous.

Tubes minute, $2 \mathrm{~mm}$. long.

Tubes large, $1.5^{-2} \mathrm{~cm}$. long.

3. I. jamaicensis.

4. I. porrectus.

5. I. leprosus.

Spores faintly tinged with brown.

Tubes invisible to the unaided eye.

Pileus thick, azonate, margin obtuse; hymenium dull.

6. I. corrosus.

Pileus thin, zonate, margin very sharp; hymenium glistening.

Tubes visible to the unaided eye, although sometimes small.

Surface soft and spongy; hymenophores found on living shrubs, often encircling the twigs.

Surface not soft and spongy; hymenophores found on decaying trunks or branches.

Pileus very minute, $2 \mathrm{~mm}$. broad.

Pileus of medium size, $6 \rightarrow 9 \mathrm{~cm}$. broad.

9. I. pusillus.

I0. I. radiatus.

\section{INONOTUS PERTENUIS Murrill}

Pileus very thin, slightly flexible, fragile, sessile, densely imbricate, decurrent, laterally connate, conchate, 2-3 $\times 4-6 \times$ $0.1-0.2 \mathrm{~cm}$.; surface striate, hispid-squamulose, anoderm, spuriously zoned, fulvous; margin very thin, lobed, fimbriate, sharply decurved in dried specimens; context fulvous, corky, I $\mathrm{mm}$. or less thick; tubes ferruginous to fulvous, I-I.5 mm. long, mouths circular to angular, minute, 5-6 to a mm., edges thin, entire to lacerate, fragile, ferruginous to dark-fulvous; spores ovoid, smooth, pale-ferruginous, $5 \times 4 \mu$.

Found once on dead wood on El Yunque in Cuba and once in the mountains of Panama. 


\section{INoNotus FULVOMELleUS Murrill}

Pileus somewhat irregular, dimidiate to subcircular, compressed-ungulate, $3-5 \times 5^{-10} \times \mathrm{I}-3 \mathrm{~cm}$.; surface uneven, ferruginous to fulvous, slightly spuriously zonate, roughly hirsute or shaggy with dense, branched, fulvous hairs; margin usually thick, undulate, ochraceous-luteous, hispid; context radiatefibrous, luteous, shining, 3-7 mm. thick; tubes slender, 3-10 mm. long, dark-melleous within, mouths circular, 4-5 to a mm., regular, covered when young with a bright-yellow powder, edges thick, entire, flavous-melleous to melleous-fulvous; spores ovoid, thin-walled, pale-ferruginous, $8 \times 6 \mu$; cystidia dark-reddish-brown, slender, conic, hooked at the tip, Io $\mu$ thick at the base, $60 \mu$ or less in length.

Found a few times on dead wood at high elevations in the Blue Mountains, Jamaica.

\section{Inonotus JAMAICENSIS Murrill}

Pileus dimidiate to triquetrous, convex, sessile, attached by a broad base, simple or imbricate, $2 \times 3 \times 1-1.5 \mathrm{~cm}$.; surface encrusted, minutely rugose, cinereous behind, marked toward the margin with dark-brown or black zones; margin regular, of ten obtuse; context fibrous, fulvous, only a few millimeters thick; tubes I cm. long, 4 to a $\mathrm{mm}$., larger by confluence, fulvous, polygonal to irregular, edges thin, entire; spores ovoid, smooth, deep-ferruginous, I-2-guttulate, very copious, $7 \times 5 \mu$.

Found once on dead branches on the Mabess River, Jamaica.

\section{INONOTUS PORRECTUS Murrill, sp. nov.}

Pileus flabelliform, more or less imbricate, tapering behind or attached by a lateral stipitiform base, rarely slightly multiplex with a common stipe when growing on the top of the substratum, 6-8 $\times 8$-Io $\times 0.5^{-1.5} \mathrm{~cm}$.; surface uneven, zonate, radiaterugose, with rather prominent ridges near the center, glabrous, fulvous to bay; margin thin, undulate or lobed, pallid above, distinctly marked below by a narrow, yellow, sterile zone; context hard, fibrous, zonate, shining, bright-ochraceous-melleous, thin in front, reaching $I \mathrm{~cm}$. or more thick behind; tubes about $2 \mathrm{~mm}$. long, umbrinous within, mouths minute, angular, thinwalled, 5-6 to a mm., edges at first whitish or grayish, becoming umbrinous with age; spores copious, subglobose, smooth, fulvous, 4.5-5.5 $\mu$; cystidia none.

Type collected on a decaying stump on Caicos Islands, in the Bahamas, December 18, 1907, Percy Wilson 7748 (herb. N. Y. Bot. Gard.). Also collected in Louisiana by A. B. Langlois. 
5. InoNotus Leprosus (Fries) Murrill, comb. nov.

Pileus thick, pulvinate, narrowly attached and nearly circular to dimidiate, $5^{-12} \mathrm{~cm}$. broad, $3-5 \mathrm{~cm}$. thick; surface nearly smooth, azonate, short-tomentose in spots, finally glabrous, fulvous or chestnut in dried specimens; margin thin, concolorous, dark-colored when bruised; context soft, punky, isabelline to pale-umbrinous, $2 \mathrm{~mm}$. thick in front, about $\mathbf{I ~} \mathrm{cm}$. thick behind; tubes long and large, ochraceous when young, becoming reddish-fulvous when bruised, $1.5^{-2} \mathrm{~cm}$. in length, mouths alveolar, $0.5^{-2}$ to a $\mathrm{mm}$., sometimes becoming irregular and lacerate with age, edges thin, entire or at length deeply toothed; spores broadly ellipsoid, smooth, rather thick-walled, deep-fulvous, $6.5^{-7} \times 5^{-6 \mu}$; cystidia none.

Found twice in the island of St. John and once in Mexico, on dead wood. This species was formerly placed in Favolus, but recent collections show it to belong in Inonotus.

\section{INONOTUS CORROSUs Murrill}

Pileus conchate, clasping, simple or imbricate, $3 \times 5 \times 1-4$ $\mathrm{cm}$.; surface ferruginous to fulvous, furrowed and much corroded in age; margin entire, obtuse, tomentose, honey-yellow; context thick, spongy, fibrous, ferruginous, perforated by insects soon after maturity; tubes very short, only I mm. long each season, 8 to a mm., fulvous, subcylindric, edges entire, obtuse to acute; spores lenticular, smooth, pale-ferruginous, $4 \mu$ in diameter, I $\mu$ thick.

Common on decayed vines and trunks in southern Florida and the West Indies. Closely related to $I$. fruticum.

\section{Inonotus Wilsonir Murrill}

Pileus dimidiate, applanate, sessile, $2-3 \times 4-6 \times 0.5 \mathrm{~cm}$.; surface anoderm, velvety-tomentose, fulvous, marked with a few shallow, concentric furrows; margin thin, entire, concolorous, sulcate, deflexed in drying; context soft, punky, homogeneous, ferruginous-fulvous, $\mathrm{I}-3 \mathrm{~mm}$. thick, separated from the tubes by a very thin, black layer; hymenium ferruginous, glistening; tubes $\mathrm{I}-2 \mathrm{~mm}$. long, 6-9 to a $\mathrm{mm}$., isabelline within, mouths polygonal, regular, edges thin, entire; spores lenticular, smooth, pale-ferruginous, $3-4 \mu$ in diameter, I-I.5 $\mu$ thick.

Found once on dead logs in Honduras. 


\section{Inonotus FRuticum (Berk. \& Curt.) Murrill}

Pileus soft, spongy, thin, dimidiate or vertically attached, I $-2.5 \mathrm{~cm}$. broad, 2-5 $\mathrm{mm}$. thick; surface spongy-tomentose, azonate, flavous to fulvous; margin thin and undulate, becoming blackish when bruised; context flavous to ferruginous, spongy, I-3 mm. thick, with a very thin, firm, darker layer next to the tubes; tubes short, ferruginous to fulvous, about I mm. long, mouths angular to irregular, 3 to a mm., edges thin, toothed, ferruginous to fuliginous, hoary when young; spores subglobose, smooth, pale-ferruginous, $3-4 \mu$.

Occasional on living twigs of orange and oleander in Cuba.

\section{INONOTUS PUSILLUS Murrill}

Pileus sessile, convex, flabelliform, tapering to a narrow base, erumpent from lenticels, $2 \times 2 \times 0.5^{-1} \mathrm{~mm}$.; surface ferruginous to fulvous, silky-striate, subzonate, shining, margin pallid, acute, of ten depressed; context thin, fibrous, ferruginous; tubes umbrinous, comparatively large, $2-4$ to a $\mathrm{mm}$., polygonal, becoming irregular, much exceeding in length the thickness of the context, mouths at first whitish-pulverulent, dissepiments thin, entire; spores ovoid, $5 \times 3.5 \mu$, pale-ferruginous.

Found once on dead branches of Jacquinia at Manzanillo, Mexico.

\section{IO. InoNotus Radiatus (Sow.) P. Karst.}

Pileus corky to woody, imbricate, confluent, sessile, umbonate behind, especially when young, 3-5 $\times 6-9 \times 0.5^{-1} \mathrm{~cm}$.; surface radiate-rugose to very uneven, minutely velvety to glabrous, fulvous to ferruginous-fuscous or almost black behind; margin thin, pallid, undulate to lobed; context subzonate, ferruginous to dark-fulvous, I-3 mm. thick; tubes slender, grayish-umbrinous to fulvous, about $5 \mathrm{~mm}$. long, mouths angular, somewhat irregular, 3-5 to a mm., edges whitish at first, becoming darkfulvous with age, glistening, thin, fimbriate to lacerate; spores ellipsoid, luteolous, $4-6 \times 3^{-4} \mu$.

Reported twice from Cuba by Wright.

\section{PHAEOLUS Pat.}

Hymenophore large, irregular, annual, spongy to corky, epixylous; stipe simple, variously attached, wanting at times; surface of pileus anoderm, hispid; context ferruginous; tubes irregular, thin-walled; spores ellipsoid, smooth, hyaline; cystidia none. 


\section{Phaeolus sistotremoides (Alb. \& Schw.) Murrill}

Pileus spongy, circular, varying to dimidiate or irregular, 15$20 \mathrm{~cm}$. broad, $0.5^{-2} \mathrm{~cm}$. thick; surface setose-hispid to strigosetomentose and scrupose in zones, ochraceous-ferruginous to fulvous-castaneous or darker, quite uneven, somewhat sulcate, obscurely zonate; margin yellow, rather thick, sterile; context very soft and spongy, fragile when dry, sometimes indurate with age, flavous-ferruginous to fulvous, $0.3-0.7 \mathrm{~mm}$. thick; tubes short, 2-5 mm. long, flavous within, mouths large, irregular, averaging I mm. in diameter, edges thin, becoming lacerate, ochraceous-olivaceous to fuliginous, rose-tinted when young and fresh, quickly changing to dark-red when bruised; spores ellipsoid, $7-8 \times 3-4 \mu$; stipe central to lateral or obsolete, very irregular, tubercular or very short, resembling the pileus in surface and substance.

Occasional in Mexico and Cuba on trunks, stumps, and roots of various coniferous trees, causing a very serious reddish-brown rot of the roots and lower part of the trunk.

\section{COLTRICIELLA Murrill}

Hymenophore small, annual, tough, epixylous; stipe attached to the vertex of the pileus; surface of the pileus anoderm, zonate; context spongy, fibrous, ferruginous; tubes angular, one-layered, dissepiments thin; spores ellipsoid, smooth, ferruginous.

\section{Coltriciella Dependens (Berk. \& Curt.) Murrill}

Hymenophore gregarious or cespitose; pileus very small, conic, pendant, vertically attached, $\mathrm{I}-2 \mathrm{~cm}$. broad, about $\mathrm{I} \mathrm{cm}$. thick; surface cinnamon-colored, soft, elongate-striate, sericeous, subzonate; margin acute, fibrillose; context spongy, very thin, ferruginous-fulvous, $1-2 \mathrm{~mm}$. thick; tubes long, $5-8 \mathrm{~mm}$., fulvous, mouths large, angular, I-2 to a mm., smaller near the margin, edges thin, toothed, yellowish to fulvous; spores ellipsoid, smooth, ferruginous, $7-8 \times 3.5-4 \mu$; stipe central, attached at the vertex, cylindric, gradually enlarging as it approaches the pileus, about $\mathrm{I} \mathrm{cm}$. long, $\mathrm{I}-3 \mathrm{~mm}$. thick, resembling the pileus in surface and substance.

Occasional on decorticated pine wood in the Carolinas and reported on Liriodendron in Florida. It may not occur within our range but is well worth a careful search. 


\section{COLTRICIA (Micheli) S. F. Gray}

Hymenophore annual, terrestrial or humus-loving, simple, small to medium, usually circular and central-stemmed; surface anoderm, brown, zonate or azonate; context yellowish or brown, coriaceous to spongy; hymenium concolorous, covered with yellowish or whitish powder when young; tubes thin-walled, at length fimbriate; spores smooth, rounded, yellowish-brown; cystidia rarely present.

Pileus regularly circular in outline.

Pileus shining-cinnamon, strigose, striate, thin, flexible, slightly depressed, the margin often fimbriate or pseudociliate.

I. C. cinnamomea.

Pileus dull-rusty-cinnamon to hoary, velvety to glabrous, deeply depressed, the margin thicker and less fimbriate.

Tubes small, $0.5 \mathrm{~mm}$. or less in diameter.

Tubes large, I mm. in diameter.

2. C. perennis.

3. C. focicola.

Pileus irregular and variable in outline, dimidiate, reniform or spatulate, or separated into several lobes; confined to the tropics.

4. C. spathulata.

\section{Coltricia cinnamomea (Jacq.) Murrill}

Pileus coriaceous, thin, circular, umbilicate, sometimes deeply so, $\mathrm{I}-4 \mathrm{~cm}$. in diameter, $\mathrm{I}-2 \mathrm{~mm}$. thick; surface bright-cinnamon, cinereous, shining, strigose-striate, zonate; margin undulate to slightly lobed, fimbriate, concolorous; context membranous, concolorous, less than a mm. thick; tubes pale-umbrinous within, I-2 mm. long, slightly decurrent, mouths rather large, angular, ferruginous to fulvous, $2-3$ to a mm., edges thin, fimbriate-dentate, collapsing with age; spores ellipsoid, pale-yellowishbrown, smooth, 6-8 $\times 4-6 \mu$; stipe central, velvety, reddishfuscous, nearly equal, 2-4 cm. long, 3-5 $\mathrm{mm}$. thick.

Reported from Cuba by Wright.

\section{Coltricia perennis (L.) Murrill}

Pileus coriaceous, circular, infundibuliform, $3-6 \mathrm{~cm}$. broad, I.5-3 $\mathrm{mm}$. thick; surface zonate, short-tomentose, substriate, ferruginous to cinereous, the zones sometimes glabrous and chestnut-colored; margin very thin, entire to lacerate, inflexed when dry; context very thin, concolorous, scarcely a mm. thick; tubes short, grayish-umbrinous within, I-3 mm. long, mouths small, angular, 2-4 to a mm., whitish when young, becoming fulvous, edges thin, dentate to lacerate, soon collapsing; spores ovoid, smooth, pale-yellowish-brown, $4-6 \times 2-3.5 \mu$; stipe bulb- 
ous and often united with that of neighboring plants at the base, tapering upward, velvety, ferruginous to fulvous, solid, corky, 3-5 cm. long, 2-5 mm. thick.

Found once at Cuernavaca, Mexico.

\section{Coltricia focicola (Berk. \& Curt.) Murrill}

Pileus membranous, circular, umbilicate, $3-6 \mathrm{~cm}$. in diameter, $0.5^{-1} \mathrm{~cm}$. thick; surface velvety, cinnamon to cinereous, multizonate; margin thin, entire or undulate; context very thin, ferruginous to fulvous, scarcely I $\mathrm{mm}$. in thickness; tubes long, ample, ferruginous to fulvous within, 5-8 mm. long, mouths I-2 $\mathrm{mm}$. in diameter, angular, fulvous, edges thin, toothed, becoming lacerate and collapsed with age, causing the pores to appear much smaller than they really are; spores oblong-ellipsoid, smooth, pale-yellowish-brown, abundant, I-guttulate, $6 \times 3.5 \mu$; stipe central, cylindric, slightly enlarged at the base, velvety, ferruginous to fulvous, solid, corky, $2-3 \mathrm{~cm}$. long, 3-5 mm. thick.

Reported twice from Cuba by Wright, but the specimens representing one of these collections, at least, belong to a different species.

\section{Coltricia spathulata (Hook.) Murrill}

Pileus small, coriaceous, multiform, varying from dimidiate to reniform or spatulate, or separated into several distinct lobes, $0.5-\mathrm{I} .5 \times \mathrm{I}-2.5 \times 0 . \mathrm{I}-0.2 \mathrm{~cm}$.; surface narrowly zonate, silky, rarely depressed, flavous to ferruginous-fulvous with fulvous or chestnut-colored zones; margin flavous, very thin, undulate to lobed; context membranous, very thin, flavous, with a black line near the surface, scarcely a mm. thick; tubes very short, exceedingly minute, not decurrent, less than $1 \mathrm{~mm}$. long, mouths angular, stuffed when young, flavous to umbrinous, about to to a mm., edges thin, subentire; spores smooth, yellowish-brown; stipe lateral, eccentric or erect, irregular, of ten branched, velvety, solid, tough, ferruginous to fulvous, $2-5 \mathrm{~cm}$. long, 2-4 mm. thick.

Frequent throughout on soil or on dead wood.

\section{CRYPTOPORUS (Peck) Hubbard}

Hymenophore subglobose, sessile, epixylous; surface smooth, encrusted; context white, corky; tubes white, concealed at first by a volva, which is perforated at one or more points at maturity; mouths constricted, discolored; spores smooth, hyaline. 


\section{Cryptoporus volvatus (Peck) Hubbard}

Pileus simple, sessile, rarely spuriously stipitate, globose to ungulate, 2-6 cm. broad, $1.5-3 \mathrm{~cm}$. thick; surface white, sometimes slightly reddish-brown, smooth, slightly viscid or resinous when young, glabrous, marked with anastomosing depressed lines in larger specimens; margin very rounded, concolorous, smooth, produced into a volva covering the tubes, at length ruptured at I-3 points forming small rounded or irregular apertures; context soft-corky, homogeneous, white, $2-5 \mathrm{~mm}$. thick; tubes I-I.5 mm. long, isabelline to umbrinous, mouths angular, yellow with a tinge of cinnamon, 3 to a mm., edges thick, becoming thin, entire; spores oblong, hyaline or pale-flesh-colored, I I $-I_{3} \times 4-5 \mu$.

Occasional on decayed coniferous trunks in Mexico.

\section{FOMES Gill.}

Hymenophore sessile, ungulate or applanate, epixylous; surface anoderm or encrusted, sulcate, rarely zonate; context white, wood-colored, or flesh-colored, corky or woody, rarely punky; tubes cylindric, usually thick-walled, stratose; spores smooth, hyaline or subhyaline.

Context flesh-colored; light-brown in faded specimens.

Tubes I-2 mm. long each season; spores ellipsoid.

Tubes 3-5 mm. long each season; spores globose.

I. $F$, roseus.

2. F. Sagraeanus.

Context white or nearly so.

Pileus less than $3 \mathrm{~cm}$. broad.

Pileus ungulate; tubes 5-10 mm. long.

Pileus scutellate; tubes less than $2 \mathrm{~mm}$. long.

Pileus more than $3 \mathrm{~cm}$. broad.

Pileus encrusted; surface darker than the context.

Pileus thin, applanate.

Surface zonate.

Surface azonate.

Pileus thick and usually ungulate.

Marginal zone pallid to gray or brown.

Marginal zone pallid to yellowish or reddishchestnut.

3. F. turbinatus.

4. F. scutellatus.

5. F. annosus.

6. F. subferreus.

7. F. ligneus.

8. F. ungulatus.

Pileus rarely encrusted; surface concolorous with the context.

Tubes large, 2 to a mm., becoming reddish when bruised; annual strata separated by thick layers of context.

Tubes small, 5-7 to a mm., not changing to reddish when bruised.

Surface zonate.

Surface azonate.

Iо. F. Auberianus.

II. F. geotropus. 


\section{Fomes roseus (Alb. \& Schw.) Cooke}

Pileus woody, dimidiate, varying from conchate to ungulate, often imbricate and longitudinally effused, $2-4 \times 6-30 \times 0.5-3$ $\mathrm{cm}$.; surface rugose, subfasciate, slightly sulcate, rosy or fleshcolored, becoming gray or black with age; margin acute, becoming obtuse, sterile, pallid, often undulate; context floccose-fibrous to corky, rose-colored, $0.2-2 \mathrm{~cm}$. thick; tubes indistinctly stratose, I-2 mm. long each season, mouths circular, 3-4 to a mm., edges obtuse, concolorous; spores ellipsoid, smooth, thick-walled, subhyaline, $3.5 \times 6 \mu$.

Found once at Jalapa, Mexico, and a few times in the Bermudas; reported once from Cuba by Wright. It is easily confused with $F$. Sagraeanus.

\section{Fomes Sagraeanus (Mont.) Murrill}

Pileus corky to woody, dimidiate, sessile, conchate or applanate, often irregular, 4-6 $\times 5^{-10} \times 0.5^{-2} \mathrm{~cm}$.; surface anoderm, velvety, azonate, avellaneous, subsulcate, becoming glabrous, brown or black, and horny-encrusted with age; margin thin, concolorous; context corky, flesh-colored or light-brown, I-3 mm. thick; tubes indistinctly stratified, pale-rose-colored, 3-5 $\mathrm{mm}$. long each season, often found in one layer only, mouths circular, 5 to a mm., edges obtuse, entire, roseous, becoming discolored with age; spores globose, smooth, subhyaline or slightly smoky, $6 \mu$.

Frequent in many parts of the region on dead logs and stumps.

3. Fomes turbinatus (Pat.) Murrill, comb. nov.

Pileus usually conic, pendant, and vertically attached, but sometimes dimidiate-sessile and ungulate, $\mathrm{I}-2 \mathrm{~cm}$. broad and high; surface smooth or slightly sulcate, glabrous, pale-yellow to brownish-yellow, margin entire, white; context thin, softcorky, pale-isabelline; tubes very long, reaching I cm., not distinctly stratified, isabelline within, mouths minute, constricted, milk-white when young, the edges at first very obtuse; spores globose, smooth, hyaline, 4-6 $\mu$; conidia ovoid, smooth, hyaline, $12-14 \times 6-8 \mu$.

This species was originally described from Venezuela, occurring there on decayed branches. It has since been collected occasionally in Guadeloupe; at Castleton Gardens and Chester Vale, Jamaica; and on dead limbs and decayed standing trunks in western Cuba. It greatly resembles $F$. ohiensis in appearance and habit, but has much longer, smaller tubes. 


\section{Fomes scutellatus (Schw.) Cooke}

Pileus woody, dimidiate or scutellate, concave below, $0.5-0.7$ $\times$ I-I.5 $\times 0.3-0.5 \mathrm{~cm}$.; surface rugose, tuberculose, zonate, fuscous-black; margin acute, deflexed, pallid to light-brown; context woody, indistinctly zonate, isabelline, $2-3 \mathrm{~mm}$. thick; tubes indistinctly stratified, $\mathrm{I}-\mathrm{I} .5 \mathrm{~mm}$. long each season, isabelline within, mouths subcircular to rhomboid, 4 to a mm., edges rather thin, obtuse, entire, chalk-white, becoming avellaneous.

Occasional on dead deciduous wood at Jalapa, Mexico.

\section{Fomes annosus (Fries) Cooke}

Pileus woody, dimidiate, very irregular, conchate to applanate, I0-I $3 \times 5^{-8} \times 0.5^{-2} \mathrm{~cm}$.; surface at first velvety, rugose, anoderm, light-brown, becoming thinly encrusted, zonate, and finally black with age; margin pallid, acute, becoming thicker; context soft-corky to woody, white, $0.3-0.5 \mathrm{~cm}$. thick; tubes unevenly stratified, $2-8 \mathrm{~mm}$. long each season, white, mouths subcircular to irregular, $3-4$ to a $\mathrm{mm}$., edges rather thin, entire, firm, white, unchanging; spores subglobose or ellipsoid, smooth, hyaline, 5-6 $\times 4^{-5} \mu$.

Reported twice by Wright on pine logs in Cuba.

\section{Fomes SUBFERREUS Murrill}

Pileus corky to woody, conchate or applanate, imbricate, usually longitudinally effused, $3-9 \times 5^{-15} \times \mathrm{I}-2 \mathrm{~cm}$.; surface velvety, rugose-striate, slightly sulcate, black, with traces of purple, becoming glabrous and gray with age; margin subobtuse, pallid, marked with purplish-black blotches; context punky, isabelline, $0.5^{-I} \mathrm{~cm}$. thick; tubes unevenly stratified, $2-3 \mathrm{~mm}$. long each season, glistening-white, discolored in the older layers, mouths circular, 5 to a mm., edges obtuse, glistening, milk-white to avellaneous or isabelline; spores globose, smooth, hyaline, $3 \mu$.

Occasional on hardwood logs in Cuba, western Jamaica, Costa Rica, and Honduras.

\section{Fomes Ligneus (Berk.) Cooke}

Pileus very hard, applanate or ungulate, $5^{-10} \times$ I3-15 $^{-1} 4^{-8}$ cm.; surface smooth, sulcate, dark-brown or black, becoming horny-encrusted and shining with age; margin obtuse, pallid; context hard and woody, white to pale-umbrinous, $0.5^{-1} \mathrm{~cm}$. thick, extremely scanty in old specimens; tubes evenly stratified, 3-5 $\mathrm{mm}$. long each season, white, mouths minute, circular, 3-4 
to a mm., edges obtuse, white or slightly avellaneous; spores subglobose or ovoid, smooth, hyaline, $7 \times 6 \mu$.

Occasional on dead wood throughout most of tropical America.

\section{Fomes ungulatus (Schaeff.) Sacc.}

Pileus corky to woody, ungulate, 8-I5 $\times 12-40 \times 6-10 \mathrm{~cm}$.; surface glabrous, sulcate, reddish-brown to gray or black, often resinous; margin at first acute to tumid, pallid, becoming yellowish or reddish-chestnut; context woody, pallid, $0.5^{-1} \mathrm{~cm}$. thick; tubes distinctly stratified, $3-5 \mathrm{~mm}$. long each season, white to isabelline, mouths circular, $3-5$ to a mm., edges obtuse, white to cream-colored; spores ovoid, smooth, $6 \mu$.

Reported once by Wright on pine trunks in Cuba.

\section{Fomes RUBRITINCTUS Murrill}

A large perennial plant of many layers, the upper partly dead and discolored, the lower smooth, light-colored, and anoderm, with large pores, which become dark-red when bruised; pileus corky to woody, dimidiate, convex, $4^{-12} \times 5^{-15} \times 3^{-10} \mathrm{~cm}$.; surface anoderm, smooth, velvety to the touch, white or pallid, becoming dark-brown and roughened with age; margin obtuse, sterile, concolorous; context corky, becoming woody, pallid, $\mathbf{I} .5 \mathrm{~cm}$. thick; tubes unevenly stratified, the layers separated by unusually thick cushions of context, $0.5^{-1} \mathrm{~cm}$. long each season, 2 to a mm., isabelline within, becoming umbrinous in the older layers, mouths subcircular or polygonal, edges thin, isabelline, becoming dark-red when bruised; spores globose to ovoid, $5^{-7 \mu}$ long, the wall of medium thickness.

Collected once on dead trunks in Nicaragua.

\section{io. Fomes Auberianus (Mont.) Murrill}

Pileus woody, conchate to pulverulent, 4-10 $\times 7-15 \times 1-2$ $\mathrm{cm}$.; surface glabrous, rugose, radiate-striate, zonate, isabelline to light-fulvous; margin thin but usually obtuse, pallid, turning bay when bruised, ungulate to lobed, deflexed; context corky to woody, almost white, $0.5-1.5 \mathrm{~cm}$. thick; tubes distinctly stratose, 3-7 $\mathrm{mm}$. long each season, fulvous within, mouths circular to angular, minute, $6-7$ to a mm., edges thin, entire, light-bay, darker when bruised, subglistening; spores globose, $3 \mu$.

Common throughout on dead or wounded hardwood trunks. 


\section{Fomes geotropus Cooke}

Pileus woody, conchate, very thick behind, 6-12 $\times$ I0-20 $\times 2-4 \mathrm{~cm}$.; surface pruinose to glabrous, roughly rugose, anoderm, azonate, irregularly concentrically undulate, stramineous to cremeous; margin tumid, pallid, brownish when bruised, slightly undulate, deflexed; context corky, pallid to isabelline, $0.5^{-1.3} \mathrm{~cm}$. thick; tubes distinctly stratified, $0.5-1.5 \mathrm{~cm}$. long each season, pale-chestnut-colored, fading out in the older layers, mouths circular, minute, 5 to a $\mathrm{mm}$., edges thin, entire, rose-colored when fresh, becoming darker and discolored with age or when bruised.

Frequent on living and dead trunks in many parts of tropical America, causing serious decay.

\section{FOMITELLA Murrill}

Hymenophore sessile, at times semiresupinate, applanate, epixylous; surface glabrous, anoderm to encrusted, sulcate with age; context woody or slightly punky, brownish-olivaceous, rarely varying to pallid; tubes minute, cylindric, usually thickwalled, more or less stratose at maturity; spores smooth, hyaline.

Tubes 5 to a mm.

Tubes $\mathrm{I}-2$ to a mm.
I. F. supina.

2. F. fumoso-avellanea.

\section{Fomitella supina (Sw.) Murrill}

Pileus rigid, corky to woody, dimidiate, thin, sessile, imbricate, plane or concave below, 4-6 $\times 5$-10 $\times 0.5^{-1} \mathrm{~cm}$.; surface finely tomentose to glabrous, azonate, smooth or rugose, varying from white to umbrinous, often blotched with purple or entirely purplish-black behind; context corky, zonate, greenish-isabelline to olivaceous, 2-6 mm. thick; tubes normally perennial, annual in many specimens, indistinctly stratose, I-3 $\mathrm{mm}$. long each season, isabelline to grayish-umbrinous within, mouths circular, rarely elongate, 5 to a mm., edges rather thin at maturity, entire but slightly uneven, isabelline to umbrinous; spores globose, smooth, $4 \mu$.

Very common throughout on various forms of dead wood.

\section{Fomitella fumoso-avellanea (Romell) Murrill}

Pileus corky, sessile, imbricate, decurrent, applanate, convex below, triangular in section, $2-4 \times 5-10 \times \mathrm{I}-2.5 \mathrm{~cm}$.; surface anoderm, tomentose, uneven, azonate, opaque, dull-colored, avellaneous to umbrinous; margin usually thin, isabelline, rarely 
purplish-tinted, smooth, entire; context soft-corky, homogeneous, olivaceous-umbrinous, $5 \mathrm{~mm}$. thick; tubes indistinctly stratified, 3-5 $\mathrm{mm}$. long each season, avellaneous to umbrinous, mouths angular, somewhat irregular, $\mathrm{I}-2$ to a mm., edges thin, entire, umbrinous, purplish-tinted in some specimens; spores obliquely ellipsoid, smooth, 3.5-4 $\times 2 \mu$.

Occasional on dead trunks in Mexico and Central America.

\section{PYROPOLYPORUS Murrill}

Hymenophore large, perennial, epixylous, sessile, ungulate or applanate; surface sulcate, usually anoderm and often rough or rimose; context woody or punky, brown; tubes brown, cylindric, stratose, usually thick-walled; spores smooth, hyaline.

Pileus applanate.

Context red.

Context flavous to luteous.

I. $P$. albomarginatus.

Context ferruginous to fulvous.

Hymenium isabelline to fulvous.

Hymenium chestnut-brown.

Pileus ungulate.

Hymenium roseocinereous to smoky-gray.

2. P. Haematoxyli.

3. P. Baccharidis.

4. P. Robinsoniae.

Hymenium ferruginous to fulvous.

Context ferruginous; surface dark-brown, marked with narrow, black, concentric lines; tubes thinwalled, 5 to a $\mathrm{mm}$.

Context tawny; surface black, without lines and more sulcate; tubes smaller, thick-walled, 7 to a mm.

5. P. roseocinereus.

6. P. inflexibilis.

7. P. Calkinsii.

\section{Pyropolyporus albomarginatus (Lév.) Murrill}

Pileus corky to woody, dimidiate, sessile, thin and conchate or thick and applanate, $5^{-1} 5 \times 10-25 \times 1.5-5 \mathrm{~cm}$.; surface sulcatezonate, velvety or scabrous to glabrous, latericeous, paler red on the marginal zone and white on the immediate margin; context punky to corky, orange-red to cinnabar-red; tubes distinctly stratified in older specimens, minute, about 6 to a mm., whitish to dark-ferruginous, the edges entire.

This very conspicuous and beautiful species is widely distributed throughout the oriental tropics, having been found several times in the Philippine Islands. The only specimens obtained within our range were collected in British Honduras in I907. These represent the thin, conchate form of the species and are quite different from the type of Fomes lateritius Cooke, from South America, which is thick and applanate. For the 
synonymy of this species, see an article on Philippine Polyporaceae in the Bulletin of the Torrey Botanical Club for 1907.

\section{Pyropolyporus Haematoxyli Murrill}

Pileus dimidiate, thickest behind, $12 \times 14 \times 4 \mathrm{~cm}$.; surface glabrous, dark-brown, shallowly concentrically sulcate, marked with numerous darker concentric lines; margin fulvous, thin, rounded, slightly undulate; context corky to woody, indistinctly concentrically banded, honey-yellow, I cm. thick; tubes distinctly stratified, longer behind, $0.5^{-1} \mathrm{~cm}$. long each season, 6 to a mm., dull-brown, mouths polygonal, concolorous, edges obtuse, becoming thin; spores globose, rarely ovoid, thin-walled, smooth, hyaline, $3 \cdot 5^{-5} \mu$; cystidia none.

Collected once at the base of a living logwood tree at Paradise, Jamaica.

\section{Pyropolyporus Baccharidis (Pat.) Murrill}

Pileus corky-woody, turbinate-conic, vertically attached, pendulous, 3-6 $\times 3-6 \times \mathrm{I}-2 \mathrm{~cm}$.; surface densely pectinatesulcate, soft and velvety, flavous to cinnamon, becoming glabrous, blackish, and thinly encrusted; margin obtuse, flavous, tomentose, undulate; context corky, ferruginous-fulvous, 2-3 $\mathrm{mm}$. thick; tubes evenly stratified, $2-3 \mathrm{~mm}$. long each season, fulvous-cinnamon within, mouths circular, invisible to the unaided eye, ro to a mm., edges thin, entire, flavous-fuscous to chestnut-brown, slightly glistening; spores ovoid, smooth, hyaline, $6-7 \times 4-5 \mu$; cystidia none.

Rare on trunks of Baccharis in Guadeloupe and Ecuador.

\section{Pyropolyporus Robinsoniae Murrill}

Pileus dimidiate, applanate to compressed-ungulate, $5-7 \times$ 7-10 $\times$ I.5-3 cm.; surface velvety, slightly encrusted, many times sulcate, very rough, tubercular, ferruginous to fulvous, corroded and darker with age; margin obtuse, velvety, ferruginous, sterile; context woody, fulvous, $3-5 \mathrm{~mm}$. thick; tubes unevenly stratified, 3-5 mm. long each season, umbrinous within, mouths circular, minute, 4-5 to a mm., edges obtuse, entire, chestnut-brown, glistening; spores globose, smooth, hyaline, $4 \mu$; cystidia none.

Found once on the decaying roots of a tree on Monkey Hill, Jamaica. 


\section{Pyropolyporus Roseocinereus Murrill}

Pileus applanate to compressed-ungulate, often vertically attached, 5-8 $\times$ 7-14 $\times$ 2-4 cm.; surface tomentose, becoming glabrous, horny-encrusted, repeatedly zonate-sulcate, fulvous to reddish-brown; margin acute, undulate, ferruginous, tomentose; context woody, very thin, light-fulvous, I-2 mm. thick; tubes indistinctly stratified, I-2 mm. long each season, fulvous within, mouths circular, very minute, $5^{-6}$ to a $\mathrm{mm}$., edges obtuse, entire, roseocinereous to smoky-gray; spores globose to ovoid, smooth, hyaline, $5^{-6 \mu}$; cystidia none.

Found a few times on dead wood in Cuba, Jamaica, and Costa Rica.

\section{Pyropolyporus inflexibilis (Berk.) Murrill}

Pileus convex above, plane below, somewhat compressedungulate, $7 \times 12 \times 5 \mathrm{~cm}$.; surface glabrous, horny-encrusted, dark-brown, concentrically sulcate, marked with narrow black concentric lines; margin rounded, yellowish-brown, sterile; context hard, concentrically banded, ferruginous, $2 \mathrm{~cm}$. thick behind; tubes indistinctly stratified, $0.5^{-1} \mathrm{~cm}$. long each season, 5-6 to a $\mathrm{mm}$., drab-colored within, mouths polygonal, concolorous, edges thin, acute, entire; spores globose to ovoid, smooth, thin-walled, hyaline, 3.5-4 $\times 4 \mu$; cystidia none.

Known from a few collections on dead trunks in the mountains of Jamaica, as well as in Brazil.

\section{Pyropolyporus Calminsin Murrill}

Pileus woody throughout, ungulate, ro $\times$ 10 $\times$ ro $\mathrm{cm}$.; surface glabrous, dark-brown to black, marked with rather shallow concentric furrows, the crust thin, horny, never rimose; margin rounded, concolorous with the hymenium; context very hard, woody, fulvous, I cm. thick; tubes in many indistinct layers, slender, minute, 7 to a mm., fulvous, mouths nearly circular, edges obtuse, entire; spores ovoid, smooth, hyaline, 6-8 $\times 4-5 \mu$.

Known from a few collections on living trunks of live oak and black oak in Florida and North Carolina, and doubtless occurring on live oak in southern Florida. Fomitiporia dryophila may be a resupinate form.

\section{FULVIFOMES Murrill}

Hymenophore large, perennial, epixylous, sessile, ungulate or applanate; surface sulcate, usually anoderm and of ten rough or 
rimose; context woody or punky, brown, rarely dark-red; tubes brown, cylindric, stratose, usually thick-walled; spores smooth, ferruginous or fulvous.

Pileus thin or of moderate thickness, conchate to applanate, never truly ungulate.

Context ferruginous to fulvous.

Surface soon covered with a thick, glabrous, horny, dark crust.

Tube-layers very prominent, tubes stuffed with white mycelium.

Tube-layers distinct but not prominent, tubes not conspicuously stuffed.

Dissepiments thick; hymenium concolorous with the context; surface not rimose with age.

Dissepiments thin; hymenium at length darker than the context; surface rimose in age.

Surface anoderm or slightly encrusted, not becoming rimose.

Surface conspicuously tomentose, soft to the touch; tubes short, fulvous within.

Tubes $5^{-6}$ to a $\mathrm{mm}$.

Tubes very minute, 8-1o to a $\mathrm{mm}$.

Surface soon encrusted, hard.

Tubes avellaneous within, distinctly stratified.

Tubes fulvous within, not distinctly stratified.

Surface becoming blackish and very rimose.

Context flavous to luteous.

Surface conspicuously fibrose-lacerate.

Surface inconspicuously tomentose or glabrous.

Pileus very large, $15-30 \mathrm{~cm}$. broad; context marked with interlacing lines of a lighter color.

Pileus 8-12 cm. broad; context homogeneous.

Pileus thick, ungulate.

Surface not becoming conspicuously rimose.

Pileus 2 $-5 \mathrm{~cm}$. broad.

Pileus 8-I $5 \mathrm{~cm}$. broad.

Margin only slightly obtuse.

Margin very obtuse and rounded.

Context flavous-ferruginous.

Context fulvous.

Surface soon becoming very conspicuously rimose.

Pileus very large, $12 \mathrm{~cm}$. or more broad, margin rounded.

Pileus scarcely attaining $12 \mathrm{~cm}$. in width, margin angular.

Tubes large for the genus, 3-4 to a mm., thinwalled; spores $7 \times 9 \mu$; cystidia none.

Tubes 5-7 to a mm., thick-walled.

I. $F$. grenadensis.

2. F. pseudosenex.

3. F. jamaicensis.

4. F. linteus.

5. F. subpectinatus.

6. F. calcitratus.

7. F. melleicinctus.

8. F. yucatanensis.

9. F. sarcitus.

10. F. extensus.

II. F. sublinteus.

12. F. hydrophilus.

13. F. troyanus.

I4. F. Swieteniae.

15. F. cinchonensis.

16. F. Cedrelae.

17. F. Underwoodii.

I8. $F$. dependens. 
I. Fulvifomes grenadensis Murrill, comb. nov.

Pileus hard, applanate, nearly circular to dimidiate, narrowly attached and considerably thicker behind, 5-12 $\times 7-15 \times 1.5-4$ $\mathrm{cm}$.; surface rough, rugose, closely furrowed, tomentose, hornyencrusted, fulvous to dark-chestnut-brown or black; margin obtuse, rounded in young plants, tomentose, fulvous, rather thin in large specimens; context hard, fulvous, slightly lustrous, regularly zonate, 5-7 $\mathrm{mm}$. thick; tubes very evenly and very distinctly stratified, the strata separated by very thin layers of context, I-I.5 mm. long each season, fulvous, filled with white mycelium, mouths circular, minute, 5 to a mm., edges thick, entire, fulvous to fuliginous; spores globose, smooth, lightyellowish-brown, 3-4 $\mu$; cystidia none.

Found once on dead wood in Grenada.

2. Fulvifomes pSeudosenex Murrill, comb. nov.

Pileus very hard, dimidiate, applanate, usually concave below, I 2-20 $\times$ I5-30 $\times$ 2-3 cm.; surface glabrous, horny-encrusted, subshining, repeatedly slightly sulcate, chestnut-brown to nearly black; margin thin, subobtuse, undulate to lobed, ferruginous; context woody, ferruginous, 3-5 $\mathrm{mm}$. thick; tubes evenly stratified, at times separated by layers of context, 2-3 $\mathrm{mm}$. long each season, fulvous within, mouths circular, very minute, 6 to a mm., edges obtuse, entire, becoming dark-fulvous; spores globose, smooth, fulvous, $4-5 \mu$; cystidia none.

Occasional on decayed trunks in Mexico and Nicaragua.

\section{Fulvifomes Jamaicensis Murrill, comb. nov.}

A rather fan-shaped plant, the upper layers of which are dead and much cracked and roughened, while the layers added below are smaller each succeeding year; pileus applanate, much thicker behind, $8 \times \mathrm{I} 3 \times 0.5-5 \mathrm{~cm}$.; surface uneven, radiately rimose, dark-brown to black; margin ferruginous, velvety, acute, becoming black, spreading and lobed, projecting $\mathrm{I}-2 \mathrm{~cm}$. beyond the new layers; context woody, fulvous, $0.5^{-1} \mathrm{~cm}$. thick; tubes stratified, separated by thin annual layers of context, $0.2-0.7$ $\mathrm{cm}$. long each season, 7 to a mm., fulvous within, mouths rounded or polygonal, hoary when young, edges thin, acute, entire; spores globose or subglobose, thin-walled, smooth, pale-golden-yellow (probably darker in age), 5-7 $\mu$.

Found once on old stumps of Psidium near Port Antonio, Jamaica. 
4. Fulvifomes linteus (Berk. \& Curt.) Murrill, comb. nov.

Pileus hard, heavy, dimidiate to nearly circular, applanate, 7-10 $\times 8$ - I5 $\times$ 3-5 cm.; surface villose-tomentose, castaneous with a fulvous tint, densely sulcate, becoming darker and slightly rimose with age; margin obtuse, melleous to fulvous, tomentose; context corky to woody, melleous-ferruginous with silky luster, slightly zonate, bounded above by a dark, horny layer, 3-6 mm. thick; hymenium umbrinous with a grayish tint; tubes indistinctly stratified, 3-5 $\mathrm{mm}$. long each season, fulvous-chestnut within, mouths circular, minute, 6 to a mm., edges rather thin, entire, ferruginous to fulvous; spores subglobose, smooth, ferruginous, $3-4 \mu$; cystidia cuspidate, dark-fulvous, $60-120 \times 10-$ $20 \mu$.

Occasional on dead trunks of trees in Mexico and Nicaragua. An interesting feature of this species is the appearance under a pocket lens of numerous large, brown, pointed cystidia on the broken surfaces of the young context.

\section{Fulvifomes subpectinatus Murrill, comb. nov.}

Pileus thin, conchate, dimidiate to flabelliform, imbricate, laterally connate, of ten decurrent and effused behind, 2-4 $\times$ 4-8 $\times 0.3-0.5 \mathrm{~cm}$.; surface conspicuously tomentose, repeatedly slightly sulcate, longitudinally furrowed at times, chestnutbrown, with a thin black crust beneath a heavy coating of tomentum; margin subacute, sterile, tomentose, ferruginous, undulate to lobed; context very thin, ferruginous to fulvous, I-2 $\mathrm{mm}$. thick; tubes indistinctly stratified, I-2 $\mathrm{mm}$. long each season, fulvous within, mouths circular, extremely minute, 9-1o to a mm., edges thin, entire, glistening, dark-melleous to ferruginous; spores globose, smooth, light-brown, $2 \mu$; cystidia none.

Occasional on dead wood in Cuba, Jamaica, and Mexico.

6. Fulvifomes Calcitratus (Berk. \& Curt.) Murrill, comb. nov.

Pileus dimidiate, applanate to compressed-ungulate, 4-10 $\times$ 6-12 $\times$ I-3 cm.; surface tomentose, zonate, sulcate, umbrinous, becoming glabrous, darker and horny-encrusted with age; margin acute to subobtuse, sterile, ferruginous to fulvous; context woody, melleous to subferruginous with silky luster, 3-10 $\mathrm{mm}$. thick; tubes distinctly stratified, often separated by thin layers of context, 4-6 mm. long each season, avellaneous within, mouths subcircular, minute, 7 to a $\mathrm{mm}$., edges rather thin, melleous when 
young, becoming avellaneous; spores globose, smooth, brown, $7 \mu$; cystidia conic, ventricose, brown, scanty, $15^{-20} \times 5^{-6} \mu$.

Occasional on dead wood in Jamaica, Cuba, and Porto Rico.

7. Fulvifomes melleicinctus Murrill, comb. nov.

Pileus woody, dimidiate, sessile, convex above, plane below, $3 \times 5^{-6} \times 1.5 \mathrm{~cm}$.; surface subglabrous to glabrous, subzonate, black or dark-bay behind, melleous on the margin, nearly smooth, slightly rimose on drying or with age, margin entire, fertile, acute or slightly obtuse; context light-fulvous, shining, faintly zonate, woody, about I cm. thick; tubes slender, fulvous within, 3-5 $\mathrm{mm}$. long, not distinctly stratified, mouths small, subcircular, 3-4 to a mm., edges rather thick, obtuse, isabelline-umbrinous, glistening; spores globose to subglobose, smooth, fulvous, $4-6 \mu$; cystidia none.

Collected once on dead wood near Tehuantepec, Mexico.

8. Fulvifomes yucatanensis Murrill, comb. nov.

Pileus dimidiate, applanate, thickest behind, 7-9 $\times 9-12 \times$ 2-3 cm.; surface clothed at first with tawny tomentum, becoming very dark brown or black and uniformly tubercular and broken into small areas by numerous, shallow, concentric furrows and radial cracks; margin narrow, acute, velvety, fulvous; context hard, fulvous, $0.5 \mathrm{~cm}$. thick; tubes rather indistinctly stratified, $0.25 \mathrm{~cm}$. long each season, 7 to a mm., fulvous, mouths circular, punctate, edges thick, obtuse; spores globose or subglobose, smooth, pale-yellowish-brown, $3 \cdot 5-5 \mu$; cystidia thick at the base, pointed, I 7-35 $\mu$.

Occasional throughout most of the region on dead trunks and stumps.

9. Fulvifomes sarcitus (Fries) Murrill, comb. nov.

Pileus hard, thin, applanate, imbricate, narrowly attached, $8 \times$ I $4 \times 0.7 \mathrm{~cm}$.; surface slightly sulcate, umbrinous to fuliginous, fibrose-lacerate, subsquamose, becoming almost smooth, with black horny crust; margin subacute, umbrinous, hispid, undulate to lobed; context hard, ferruginous, I-2 mm. thick, becoming fulvous and streaked with white in age; tubes indistinctly stratified, $\mathrm{I}-2 \mathrm{~mm}$. long each season, ferruginous within, walls circular, 5-6 to a mm., edges obtuse, entire, melleous to pale-fulvous; spores globose, smooth, very light brown, $5 \mu$; cystidia none.

Collected once on the island of St. John, West Indies, growing on dead wood. 
10. Fulvifomes extensus (Lév.) Murrill, comb. nov.

Pileus corky to woody, dimidiate to flabelliform, applanate, 10-20 $\times$ 15-30 $\times$ I-3 cm.; surface velvety, thinly encrusted, rugose, zonate, sulcate, fulvous to castaneous, becoming dark and rimose with age; margin thin, acute to subobtuse, sterile, velvety, isabelline to fulvous, undulate to lobed; context corky to woody, yellow, slightly zonate, becoming darker, with dendroid markings, in age, $0.5^{-1} \mathrm{~cm}$. thick; tubes evenly and distinctly stratified, 2-4 mm. long each season, fulvous, mouths circular, minute, 5 to a mm., edges obtuse, entire, ochraceous to fulvous; spores globose, smooth, fulvous, 4-5 $\mu$; cystidia few, ventricose, fulvous, about $15 \times 7 \mu$.

Common throughout on decayed trunks of various trees. The type at Paris from Guadeloupe is well preserved, although the color of the context is not quite so bright as in fresh specimens.

II. Fulvifomes sublinteus Murrill, comb. nov.

Pileus corky to soft-woody, dimidiate, applanate, 6-8 $\times$ 7-10 $X I-2 \mathrm{~cm}$.; surface finely tomentose to glabrous, very thinly encrusted, smooth, almost free from markings of any kind, light-bay to almost black; margin thin, rarely lobed, tomentose, ferruginous; context corky, slightly zonate, melleous, 3-Io $\mathrm{mm}$. thick; tubes distinctly stratified, separated by layers of context, 2-6 mm. long each season, umbrinous within, mouths subcircular, 7 to a mm., edges thin, entire, becoming umbrinous; spores globose, smooth, brown, $5 \mu$; cystidia none.

Found once on decayed wood of mesquite at Tepolobampo, Mexico.

12. Fulvifomes HYDROPHILUS Murrill, comb. nov.

Pileus small, woody, ungulate, usually laterally attached, $2-3 \times 2-4.5 \times 2-3.5 \mathrm{~cm}$.; surface uneven, rugose, many times sulcate, bay to chestnut with fuliginous lines, finely tomentose to nearly glabrous, horny-encrusted; margin subobtuse, melleous, entire; context scarcely I cm. thick, woody-punky, deep-ferruginous to fulvous; hymenium glistening, melleous when looked at from the side, olivaceous-umbrinous when viewed perpendicularly; tubes indistinctly stratified, fulvous with a grayish tint, 2-3 mm. long each season, mouths circular, 4-5 to a mm., edges obtuse, entire; spores subglobose, smooth, fulvous, uniguttulate, copious, thick-walled, $3.5-4.5 \mu$; cystidia none.

Collected twice at Morce's Gap, Jamaica, on dead standing saplings. 
13. Fulvifomes troyanus Murrill, comb. nov.

Pileus woody, horny-encrusted, ungulate, rarely compressedungulate, usually plane below, sessile either by the vertex or laterally, 5-8 $\times$ 8-II $\times 3-5 \mathrm{~cm}$; surface many times concentrically sulcate, slightly rimose in very old specimens, bay to nearly black, glabrous even when young; margin slightly obtuse, entire or slightly undulate, ferruginous, sterile, slightly velvety; context woody, hard, about I cm. or less thick, fulvous, penetrated by dendroid markings of a black, horny appearance; tubes rather distinctly stratified, avellaneous-umbrinous, about $3 \mathrm{~mm}$. long each season, mouths minute, about 8 to a $\mathrm{mm}$., circular, fulvous, almost castaneous when young, edges obtuse, entire; spores globose, smooth, pale-yellowish, 3-4 $\mu$.

Collected twice in Troy and Tyre, Jamaica, on a dead log.

\section{Fulvifomes Swieteniae Murrill, sp. nov.}

Pileus woody, ungulate, ro $\times$ I5 $\times 8 \mathrm{~cm}$.; surface at first velvety, scarcely encrusted, becoming slightly rimose, rough, and uneven, fulvous to chestnut, margin very obtuse and rounded, ferruginous or isabelline, at length fulvous; context corky to woody, zonate, ferruginous, becoming darker with age; tubes indistinctly stratified, 5-10 $\mathrm{mm}$. long each season, fulvous within, mouths circular to slightly angular, thin-walled, 5-6 to a mm., the edges fulvous when viewed from the side and chestnut-colored when viewed perpendicularly; spores globose, smooth, fulvous, about $4 \mu$ in diameter; cystidia none.

Type collected on a mahogany stump in a dense virgin forest in Alto Cedro, Cuba, March 19-20, 1905, F. S. Earle \& $W . A$. Murrill 545 (herb. N. Y. Bot. Gard.). Also collected at Gold Rock, Acklin's Island, Bahamas, in the winter of $1905-6, L . J$. K. Brace 4389 . Better specimens are desired.

\section{I5. Fulvifomes cinchonensis Murrill, comb. nov.}

Pileus woody, triquetrous, very thick and broadly attached behind, convex, 5-7 $\times 8-12 \times 7-10 \mathrm{~cm}$.; surface horny-encrusted, glabrous, smooth, obscurely zonate, slightly sulcate, umbrinous to bay; margin very obtuse, ferruginous, slightly undulate, sterile; context rather hard, fulvous, zonate in recent layers, $0.5^{-2} \mathrm{~cm}$. thick; tubes indistinctly stratified, avellaneous when young, becoming avellaneous-umbrinous, rather long and slender, sometimes $5 \mathrm{~mm}$. or more long during a season, mouths minute, stuffed when young, circular, 5 to a mm., avellaneousumbrinous, edges obtuse, entire; spores subglobose, smooth, 
pale-ferruginous, uniguttulate, $4 \mu$; cystidia ferruginous-fulvous, pointed, ventricose, scanty, about $20 \mu$ long and 5-7 $\mu$ thick at the base.

Collected once at Cinchona, Jamaica, on dead standing hardwood trunks in a dense virgin forest.

\section{i6. Fulvifomes Cedrelae Murrill, comb. nov.}

Pileus compressed-ungulate, broadly attached, plane below, 7-12 $\times 8-25 \times 3-7 \mathrm{~cm}$.; surface tomentose, deeply sulcate, fulvous, becoming very rough, rimose, and black with age; margin obtuse, ferruginous to fulvous, tomentose, smooth; context corky to woody, 5-10 mm. thick, fulvous; tubes evenly stratified, $2-3 \mathrm{~mm}$. long each season, fulvous within, mouths circular, invisible to the unaided eye, $5^{-6}$ to a mm., edges obtuse, entire, ferruginous to fulvous; spores globose, smooth, goldenbrown, 5-6 $\mu$.

Found once on a living trunk of Cedrela odorata in Jamacia.

17. Fulvifomes Underwoodir Murrill, comb. nov.

Pileus broadly ungulate, attached by a narrow base, concave below, $7 \times 14 \times$ I $\mathrm{cm}$.; surface many times concentrically furrowed, rimose, uniformly dark-brown to black; margin fulvous, acute or somewhat obtuse, velvety, undulate, marked with narrow zones; context hard, fulvous to dark-brown, very thin, less than $0.5 \mathrm{~cm}$; t tubes distinctly stratified, $0.5-1.5 \mathrm{~cm}$. long each season, 3-4 to a mm., brown within, mouths darker, circular or polygonal, edges acute, entire; spores ellipsoid, smooth, thinwalled, light-yellowish-brown, 8-9 $\times 5.5-7 \mu$; cystidia none.

Found twice on dead wood in Porto Rico.

\section{Fulvifomes Dependens Murrill, comb. nov.}

Pileus very hard, ungulate, attenuate and vertically affixed behind, $5^{-10} \times$ IO-I $^{-10} \times 5^{-10} \mathrm{~cm}$.; surface black, very rough and rimose, horny-encrusted, repeatedly slightly sulcate; margin abruptly acute, rarely rounded, velvety, ferruginous, undulate; context hard, woody, fulvous, very thin and inconspicuous; tubes indistinctly stratified, 2-3 $\mathrm{mm}$. long each season, ferruginous within, becoming dark-fulvous in the older layers, mouths circular, minute, 5-6 to a mm., edges obtuse, entire, fulvous to chestnut-brown, glistening; spores subglobose, smooth, subfulvous, 4-7 $\mu$ long; cystidia none.

Common in the Bahamas, Cuba, and Porto Rico on living and 
dead trunks of Guaiacum, Acacia, and certain other hardwood trees, doubtless causing a serious heart-rot.

\section{I. PORODAEDALEA Murrill}

Hymenophore large, perennial, epixylous, sessile, conchate to ungulate; surface anoderm, sulcate, usually rough; context brown and woody; tubes concolorous, rarely in distinct layers, the hymenium varying from porose to daedaleoid; spores smooth, hyaline at maturity, becoming brownish with age; cystidia conspicuous.

\section{Porodaedalea Pini (Thore) Murrill}

Pileus hard, typically ungulate, conchate or effused-reflexed in varieties, often imbricate, $5^{-8} \times 7-12 \times 5^{-8} \mathrm{~cm}$., smaller in varieties; surface very rough, deeply sulcate, tomentose, tawnybrown, becoming rimose and almost black with age; margin rounded or acute, tomentose, ferruginous to tawny-cinnamon, entire, sterile in large specimens; context soft-corky to indurate, ferruginous, 5-10 $\mathrm{mm}$. thick, thinner in small specimens; tubes stratified, white to avellaneous within, becoming ferruginous at maturity and in the older layers, $5 \mathrm{~mm}$. long each season, much shorter in thin specimens, mouths irregular, circular or daedaleoid, often radially elongate, averaging $\mathrm{I}$ to a mm., edges ferruginous to grayish-umbrinous, glistening when young, rather thin, entire; spores subglobose, smooth, hyaline at maturity, becoming brownish with age, 5-6 $\times 3-4 \mu$; cystidia abundant, short, 25-35 $\times 4-6 \mu$.

This species attacks living trunks of conifers in temperate regions, causing a very serious heart-rot. It may be expected to occur in the pine forests of tropical America, especially at high elevations.

\section{NIGROFOMES Murrill}

Hymenophore large, perennial, epixylous, sessile; context woody, purple; tubes cylindric, stratose, thick-walled, black; spores ovoid, smooth, hyaline.

\section{Nigrofomes melanoporus (Mont.) Murrill}

Pileus very hard, heavy, conchate to applanate, dimidiate, decurrent, 6-Io $\times$ I0-I5 $\times$ I-2 cm.; surface tomentose, nearly smooth, fuliginous to black, becoming glabrous, shining, several times sulcate and often rough and tuberculose with age; margin usually deflexed, at least when dry, sterile, rounded, rarely acute, 
finely tomentose, castaneous-umbrinous, becoming bay when bruised; context hard, fibrous, zonate, lustrous, dark-reddishviolet to chestnut-colored, $5 \mathrm{~mm}$. thick, much thinner in old specimens; tubes distinctly stratified, 3-4 mm. long each season, blackish to black the first year, avellaneous in the older layers, mouths circular, minute, $5^{-6}$ to a mm., edges thick, entire, umbrinous, becoming angular, thin, and dark-smoky-black with age; spores ovoid, smooth, hyaline.

Occasional throughout on dead or diseased trunks of trees.

\section{ELFVINGIELLA Murrill}

Hymenophore large, epixylous, sessile, applanate or ungulate; surface sulcate, horny-encrusted; context brown, punky; tubes brown, cylindric, stratose, thick-walled; spores smooth, hyaline or subhyaline.

I. Elfvingiella fasciata (Sw.) Murrill, comb. nov.

Pileus hard, dimidiate, applanate to ungulate, convex above, 7-10 $\times 8$-15 $\times 2-6 \mathrm{~cm}$.; surface finely tomentose, at length glabrous, concentrically sulcate, at first mole-colored, changing to umbrinous, and finally avellaneous with black fasciations; margin acute to obtuse, isabelline, sterile, undulate or entire; context punky, thin, ferruginous to fulvous, zonate, 3-5 $\mathrm{mm}$. thick; tubes indistinctly stratified, 5-10 $\mathrm{mm}$. long each season, avellaneous within, mouths circular, minute, 4-5 to a mm., edges obtuse, avellaneous to umbrinous, becoming darker when bruised; spores subglobose, smooth, light-brown, $5^{-7} \mu$.

Common throughout on dead trunks of various trees. The validity of the specific name used is doubtful.

\section{ELFVINGIA P. Karst.}

Hymenophore large, epixylous, sessile, applanate or ungulate; surface sulcate, horny-encrusted; context brown, punky; tubes brown, cylindric, stratose, thick-walled, mouths whitish or yellowish when young; spores brown; conidia present in most species on or near the surface of the pileus.

Pileus thick at maturity, crust smooth and very hard; hymenium plane; margin truncate, very smooth, often laccate.

I. E. tornata.

Pileus thin, crust radially wrinkled, thinner; hymenium concave; margin undulate, never laccate.

2. E. Lionnetii. 


\section{Elfvingia tornata (Pers.) Murrill}

Pileus hard, dimidiate, sessile or spuriously stipitate, applanate, thin, very large, plane below, $10-20 \times 15-30 \times \mathrm{I}-5 \mathrm{~cm}$.; surface horny-encrusted, sulcate, glabrous, slightly tuberculose, conidiabearing, opaque to subshining, of ten fasciate with black bands, subspadiceous to fulvous; margin smooth, sterile, of ten laccate, subacute, of ten becoming truncate; context floccose with harder fibers, zonate, fulvous to bay, with whitish markings in old specimens, 5-10 mm. thick, very thin in large specimens; tubes indistinctly stratified, $5^{-8} \mathrm{~mm}$. long each season, not separated by layers of context, dark-umbrinous within, mouths circular, not stuffed when young, often covered near the margin with resin, 4 to a mm., edges obtuse to acute, entire, pallid to umbrinous; spores broadly ellipsoid, truncate, very dark yellowish-brown, abundantly and roughly echinulate, $7-8 \times 5^{-6} \mu$.

Extremely common throughout the tropics on decayed logs and stumps.

\section{Elfvingia Lionnetil (Rolland) Murrill}

Pileus thin, applanate, $8-\mathrm{I} 2 \times \mathrm{I} 5-20 \times \mathrm{I}-3 \mathrm{~cm}$; surface horny-encrusted, profoundly radiate-rugose, zonate-fasciate, tuberculose, spadiceous-umbrinous to fuliginous; margin pallid, sterile, undulate, obtuse, reddish-brown when bruised; context floccose, hard, tawny-bay, 3-5 mm. thick; tubes indistinctly stratified, 6-10 $\mathrm{mm}$. long each season, not separated by layers of context, chestnut-colored within, umbrinous-tinted, especially in the older layers, mouths circular to polygonal, whitish-stuffed when young, 4-5 to a mm., edges obtuse, very thin, denticulate, white or cream-colored to umbrinous or bay, chestnut-colored when bruised; spores ovoid, smooth, fulvous, truncate at the base, $8 \times 5 \mu$.

Occasional on tree trunks in Nicaragua and Panama.

\section{AMAURODERMA Murrill}

Hymenophore large, epixylous, stipitate, the stipe often much elongate; surface smooth, encrusted, not varnished; context pallid or brown, punky to fibrous; tubes cylindric, concolorous, the mouths usually light-colored at first; spores ovoid or globose, brown.

Pileus $3 \mathrm{~cm}$. or less in breadth; stipe slender, less than $5 \mathrm{~mm}$. thick.

Context bay.

I. A. renatum.

Context white to avellaneous. 
Stipe $2 \mathrm{~cm}$. long; pileus orbicular, nodding.

Stipe $10 \mathrm{~cm}$. long; pileus reniform, erect.

Pileus over $3 \mathrm{~cm}$. broad; stipe over $5 \mathrm{~mm}$. thick.

Stipe lateral, ascending.

Stipe less than $5 \mathrm{~cm}$. long.

Pileus $10 \mathrm{~cm}$. or more broad; tubes 5 to a mm.; plants cespitose.

Pileus $5 \mathrm{~cm}$. or less broad; tubes 8 to a mm.; plants simple.

Stipe $15 \mathrm{~cm}$. long.

Stipe central, erect.

Hymenium white to avellaneous; stipe $15 \mathrm{~cm}$. long.

Hymenium rich-yellow; stipe less than ro $\mathrm{cm}$. long.

Pileus $30-50 \mathrm{~cm}$. broad; stipe over $5 \mathrm{~cm}$. thick.
2. A. nutans.

3. A. subrenatum.

\section{Amauroderma renatum (Berk.) Murrill}

Pileus lateral, very thin, rigid when dry, fan-shaped to reniform, attenuate behind, $\mathrm{I}-2 \times \mathrm{I} .5-3 \times 0 . \mathrm{I}-0.2 \mathrm{~cm}$.; surface silky, zonate, slightly rugose, castaneous, becoming glabrous and avellaneous-isabelline with age; margin very thin, pallid, entire to undulate or lobed; context punky, bay, homogeneous, $0.5^{-1} \mathrm{~mm}$. thick; tubes not stratified, though apparently reviving, I-I.5 mm. long, murinous to umbrinous within, mouths hexagonal, pure-white at first, becoming umbrinous with age, 5 to a mm., edges at first very thick, becoming thinner, entire; stipe very long, erect or somewhat flexuose, laterally attached, slightly expanding above, frequently branched either near the middle or at the apex, avellaneous to umbrinous, opaque, finely tomentose, fulvous and floccose within, 10-20 cm. long, $\mathrm{I}-3 \mathrm{~mm}$. thick.

Occasional in Nicaragua and Jamaica, usually attached to buried dead wood.

\section{Amauroderma nutans (Fries) Murrill}

A small plant with slender recurved stipe, having the habit of Hydnum auriscalpium; pileus corky, orbicular, convex above, concave below, $2 \times 2 \times 0.3-0.5 \mathrm{~cm}$.; surface smooth, pallid to bay-black, dull-avellaneous in type specimens, abruptly sulcate, radiate-rugose; margin thin, acute, pallid, entire, sterile; context floccose, becoming indurate, very thin, white to avellaneous, scarcely a mm. thick; tubes annual, $2-3 \mathrm{~mm}$. long, pallid to avellaneous within, young and but partially developed in type specimens, mouths angular, minute, $8-9$ to a mm., pallid to avellaneous, edges thin, entire; stipe lateral, umbonate-affixed, long, slender, flexuose, twisted as though twining, recurved at the 
apex, pallid to chestnut, encrusted, dull-avellaneous in type specimens, $2 \times 0.4-0.8 \mathrm{~cm}$.

Found once on dead trunks at San José, Costa Rica.

\section{Amauroderma subrenatum Murrill}

Pileus lateral, reniform, convex above, concave below, rigid when dry, $2 \times 3 \times 0.2 \mathrm{~cm}$.; surface glabrous, rugose, slightly zonate, abruptly shallowly concentrically sulcate, avellaneous to umbrinous, depressed behind; margin finely tomentose, zonate, pallid, rather thick; context white, thin, fibrous, $1 \mathrm{~mm}$. thick; tubes not stratified, pale-isabelline when dry, I-I.5 mm. long, mouths circular to hexagonal, regular, 4-5 to a mm., edges thick, entire, pale-avellaneous; stipe long, slender, slightly flexuose at the base, erect above, cylindric, equal, laterally attached, simple, umbrinous, opaque, firm and white within, Io $\mathrm{cm}$. long, $2 \mathrm{~mm}$. thick.

Found once on the ground in British Honduras.

\section{Amauroderma coffeatum (Berk.) Murrill}

Pileus solid, corky, suborbicular, oblique, cespitose, $18-20 \mathrm{~cm}$. broad; surface smooth, thinly encrusted, opaque, reddish-brown; margin pallid to brown, obtuse; context corky, pallid, paleisabelline in dried specimens; tubes decurrent, pallid, mouths subcircular, 5 to a mm., darker than the context, edges obtuse, entire; stipe laterally attached, elongate, cylindric, subequal, obscure-brown, mixed with coffee-color, pruinose, pallid and soft-corky, becoming fistulose, $3.5 \mathrm{~cm}$. long, I cm. thick.

Found once on decaying trunks in the island of St. Vincent, West Indies.

\section{Amauroderma regulicolor (Cooke) Murrill}

Pileus simple, corky, reniform, convex above, $2 \times 2.5-4 \times 0.3^{-}$ I cm.; surface silky, subzonate, smooth, purplish-fuscous; margin subacute, more obscure; context fulvous, punky, homogeneous, I-3 mm. thick; tubes not stratified, 3-5 mm. long, grayishumbrinous within, mouths hexagonal at maturity, 8 to a mm., grayish-fuscous, lighter when young, edges thin, entire; stipe lateral, ascending, contorted, fuscous, slightly tomentose, opaque, 4-8 cm. long, 8-12 mm. thick.

Found a few times on decaying roots of deciduous trees in Cuba. 


\section{Amauroderma avellaneum Murrill}

Pileus rather thick, flabelliform, laterally attached, $6 \times 6 \times \mathrm{I}$ $\mathrm{cm}$.; surface pulverulent to glabrous, very rugose, colliculose behind, slightly sulcate, thinly encrusted, opaque, avellaneous with faint reddish-brown zones; margin pallid, acute, lobed, pulverulent; context corky with horny radiations, pale-isabelline when dry, 3-4 mm. thick; hymenium free; tubes long and slender, not stratified, $1 \mathrm{~cm}$. long behind, shorter in front, white within, mouths circular, 4 to a mm., edges obtuse, entire, white; stipe long, ascending, subcylindric with several short aborted branches, straight, laterally attached, umbrinous, opaque, horny-encrusted, firm and nearly white within, $15 \mathrm{~cm}$. long, $1 \mathrm{~cm}$. thick.

Found once on decayed wood in Nicaragua.

\section{Amauroderma Chaperi (Pat.) Murrill}

Pileus corky to woody, regularly orbicular, plane, slightly depressed at the center, $8 \times 8 \times 1.5 \mathrm{~cm}$.; surface opaque, brownish-black, fulvous-fasciate, abundantly shallowly concentrically sulcate, with fragile crust; margin plicate-rugulose, obtuse, fertile, pallid; context punky, pale-fulvous, homogeneous, 4-5 mm. thick; hymenium free; tubes not stratified, avellaneousisabelline, $8-12 \mathrm{~mm}$. long, mouths circular or angular, regular, minute, 4-5 to a mm., edges thick, entire, avellaneous; spores exactly spheric, fulvous, echinulate; stipe central, cylindric, equal, very long, probably rooting, opaque, brownish-cinereous, palefulvous and stuffed within, $15 \mathrm{~cm}$. long, $8 \mathrm{~mm}$. thick.

Found once on dead wood in Cuba and once in Porto Rico.

\section{AMaURoderma flaviporum Murrill}

Pileus hard, rigid, circular, depressed at the center, concave below, 8-12 $\times 8-12 \times 1 \mathrm{~cm}$.; surface opaque, velvety, umbrinous with numerous bay-black zones, radiate-rugose, not sulcate; margin paler, velvety, subzonate, deflexed, at least when dry, acute, undulate to lobed; context white, corky, homogeneous, $5 \mathrm{~mm}$. thick; hymenium adnate; tubes very pale avellaneous, not stratified, 2-4 $\mathrm{mm}$. long, mouths angular, 4-5 to a $\mathrm{mm}$., stuffed when young, edges thin, entire, flavous when fresh, cremeous-melleous in dried specimens; spores ovoid, truncate, yellowish-brown, roughly echinulate, $6-7 \times 4-5 \mu$; stipe thick, central, slightly flattened, equal or expanding above, erect, opaque, horny-encrusted, velvety, dark-melleous-luteous, white and punky within, 6-8 cm. long, I.5-2 cm. thick. 
Found once on Melicocca in Jamaica and once at Santa Isabel, Porto Rico.

\section{Amauroderma Brittonil Murrill}

Pileus circular in outline, nearly plane, rather soft when fresh, becoming rigid and fragile when dry, over $30 \mathrm{~cm}$. broad in a dried condition and probably $50 \mathrm{~cm}$. when fresh; surface uneven, somewhat sulcate, castaneous to fuliginous, pelliculose, pruinose, opaque, becoming very much wrinkled and uneven on drying; context punky when dry, avellaneous, homogeneous, bounded above and below by a thin, dark layer about $2 \mathrm{~mm}$. thick; hymenium grayish when fresh, becoming brownish on drying and blackening where bruised; tubes minute, irregular, very short, less than $5 \mathrm{~mm}$., much darker than the context, being dark-fumosous in dried specimens, mouths slightly angular, 2-3 to a mm., stuffed when young, edges thin, entire, rigid; spores subglobose, slightly fuscous, finely asperulate, $7-8 \mu$; stipe subcylindric, short, central, slightly enlarged above and below, harder and more rigid than the pileus, encrusted, avellaneous, pruinose, glabrous, uneven, $10 \mathrm{~cm}$. long, $6 \mathrm{~cm}$. thick, no doubt larger in fresh specimens.

Collected once at Bachelor's Hall, Parish of St. Thomas, Jamaica, on a rotten log.

\section{GANODERMA P. Karst.}

Hymenophore large, sessile or stipitate, perennial or annual, epixylous; surface sulcate, covered with reddish-brown varnish; context punky, brown or pallid; tubes cylindric, concolorous; spores ovoid, brown.

Hymenophore stipitate, rarely varying to sessile.

Stipe very long and slender, ro- $15 \mathrm{~cm}$.

I. G. praelongum.

Stipe short to medium.

Surface of pileus narrowly multizonate.

Pileus and stipe chestnut-colored; context isabelline.

Pileus and stipe almost black; context darkfulvous.

Surface of pileus not narrowly multizonate, although more or less sulcate.

Pileus very large, $20-50 \mathrm{~cm}$. broad.

Pileus of medium size, not exceeding $10 \mathrm{~cm}$. in breadth.

Context soon indurate, very hard; margin of pileus usually lobed.

2. G. perzonatum.

3. G. subfornicatum.

4. G. pulverulentum.

5. G. stipitatum. 
Context soft and punky; margin not lobed.

Temperate species, occurring only at high elevations in the tropics.

Tropical species.

6. G. Curtisii.

Surface shining, yellowish-red to bay or dark-brown; tubes 3-5 mm. long.

7. G. subincrustatum.

Surface not shining, avellaneous to dull-castaneous; tubes 5-10 $\mathrm{mm}$. long.

8. G. argillaceum.

Hymenophore sessile, never truly stipitate.

Pileus small, less than $3 \mathrm{~cm}$. broad.

9. G. parvulum.

Pileus medium to large, $6-30 \mathrm{~cm}$. broad.

Surface scaly and glistening like mica from the excess of resin.

Surface normally laccate.

Pileus $8 \mathrm{~cm}$. thick and of very light weight.

Pileus not over $4 \mathrm{~cm}$. thick.

Surface tuberculose; hymenium cremeous when young.

Surface not tuberculose; hymenium white with a greenish tint when young.

I0. G. nitidum.

II. G. areolatum.

12. G. tuberculosum.

13. G. sessiliforme.

\section{Ganoderma PRAelongum Murrill}

Pileus corky, orbicular to fan-shaped, plane above, convex to plane below, prominently umbonate behind when young, 4-6 $\times$ 5-8 $\times$ r.5-3 cm.; surface glabrous, at length rugose and slightly sulcate, laccate, lustrous, castaneous to bay; margin ochraceous, thin, sterile, sometimes proliferous; context punky, homogeneous, isabelline with a fulvous line adjoining the tubes, $\mathbf{I}-2 \mathrm{~cm}$. thick; tubes annual, I cm. long, umbrinous within, mouths angular, 3-4 to a mm., edges thin, uneven, white to avellaneous, becoming umbrinous when bruised; spores ovoid, dark-brown, $8 \times 5 \mu$; stipe lateral, ascending, cylindric, enlarged above, 10-15 $\times 1-2$ $\mathrm{cm}$., glabrous, laccate, castaneous to nearly black, with substance like that of the pileus except for lines of white mycelium at the center.

Found once on dead wood at Alto Cedro, Cuba, and once in St. Croix.

\section{Ganoderma perzonatum Murrill}

Pileus rigid, corky, fan-shaped, thin, concave below, with a prominent umbo behind, $5^{-9} \times 4^{-8} \times 0.5^{-1} \mathrm{~cm}$.; surface at length glabrous, laccate, subshining, thinly encrusted, 2-3 times shallowly sulcate, densely zonate, the zones conspicuously marked with white and bay; margin subobtuse, free behind, undulate to lobed, shining-latericeous to bay, often forming a border beneath 
encircling the hymenium; context soft-corky, multizonate, avellaneous, fulvous next to the tubes, 3-5 $\mathrm{mm}$. thick; tubes indistinctly stratified, 3-5 mm. long each season, avellaneous within, becoming grayish in the older layers because of the white mycelium that fills them, mouths circular to slightly angular, whitish-stuffed when young, regular, 3-4 to a mm., edges nearly white to grayish-avellaneous, finally umbrinous with age; spores ovoid, brown, $8 \times 6 \mu$; stipe tubercular, umbonate-affixed, often branched, slightly darker than the pileus, $0.5^{-\mathrm{I}} \times 0.5^{-1.5} \mathrm{~cm}$.

Found once on a mango log near Santiago de las Vegas, Cuba, and once in Porto Rico.

\section{Ganoderma subfornicatum Murrill}

Pileus subcircular, rigid, nearly plane above and below, $8 \times$ 9-I I $\times$ 2-3 cm.; surface glabrous, uneven, many times concentrically sulcate, finally zonate, radiate-rugose, dark-baybrown, laccate, shining; margin truncate, concolorous, free behind; context thin, with horny radiations, slightly zonate, fulvous, 5-10 $\mathrm{mm}$. thick; tubes perennial, long and slender, $\mathrm{I} \mathrm{cm}$. in length each season, smoky-fuliginous, mouths circular, 4 to a mm., stuffed when young, edges obtuse to acute, entire, fuliginous; spores globose, smooth, brown, $4 \mu$; stipe sublateral, umbonateaffixed, scutate at the base, compressed, 3-7 $\times 1.5-2.5 \mathrm{~cm}$., expanding into the pileus, which it resembles in color, surface, and context.

Occasional on dead wood in British Honduras.

\section{Ganoderma pulverulentum Murrill}

Pileus rigid, corky, dimidiate to fan-shaped, applanate, subimbricate, usually very large, 10-20 $\times$ I $5-30 \times 2-5 \mathrm{~cm}$.; surface glabrous, laccate, lustrous, latericeous to bay, becoming dullbrown with age, broadly sulcate, azonate, uneven, at times proliferous; margin cremeous, pulverulent, subacute, rarely rounded, smooth, undulate to lobed; context punky, zonate, isabelline to umbrinous above, dark-fulvous to bay below, 5-10 $\mathrm{mm}$. thick; tubes perennial, 5-10 $\mathrm{mm}$. long each season, avellaneous within, mouths angular, 4 to a mm., citrinous-melleous, becoming darker with age, umbrinous when bruised, entirely covered at first with a creamy-white powder, which easily rubs off on the fingers, edges acute, entire; spores ovoid, brown, $9 \times 5 \mu$; stipe short, often obsolete, subcylindric, eccentric to lateral, $2-3 \times 2-3 \mathrm{~cm}$., expanding above, laccate, glabrous, bay 
to nearly black, darker than the pileus, punky and dark-fulvous within.

Occasional on dead wood in the Bahamas, Cuba, St. Thomas, and Grenada.

\section{Ganoderma stipitatum Murrill}

Pileus exceedingly hard and horny, flat and triangular or somewhat circular when young, becoming dimidiate with ungulate center and thin spreading margin, which is usually lobed and often deeply cleft, normally plane below, but frequently convex because of the upturned margin, $5 \times$ ro $\times 3 \mathrm{~cm}$.; surface glabrous, varnished, reddish-chestnut, with numerous shallow, concentric furrows, becoming shining-black and at length dull-smoky-brown; margin broad, flat, glabrous, obtuse, $3 \mathrm{~mm}$. thick, crenate to cleft, sterile portion $0.5 \mathrm{~cm}$. wide, white; context hard, woody, 0.5-I cm. thick, pale-ochraceous, with strands of dark-brown, shining, horny substance extending from the stipe to the margin in a tree-like fashion; tubes plainly though unevenly stratified, 2-4 $\mathrm{mm}$. long each season, 4-5 to a $\mathrm{mm}$., grayish-brown within, mouths circular, white or very pale yellow, edges thick, obtuse, entire; spores broadly ovoid with attenuate base, light-yellowish-brown, smooth, $3.5 \times 5 \mu$; stipe lateral, I $X$ I cm., cylindric, equal, glabrous, shining-black to dullbrown, with substance like the context, apparently absorbed or overgrown as the pileus enlarges.

Occasional on dead wood in Central America and Grenada.

\section{Ganoderma Curtisil (Berk.) Murrill}

Pileus corky to woody, reniform, convex above, concave below, 5-10 $\times 8$-I5 $\times$ I-2 cm.; surface glabrous, ochraceous to latericeous or bay, at first laccate, the varnish soon disappearing, broadly sulcate; margin obtuse to truncate, sulcate, ochraceous, entire, glabrous; context soft-corky, zonate, ochraceous above, fulvous below, $5 \mathrm{~mm}$. thick; tubes perennial, indistinctly stratified, 5-8 $\mathrm{mm}$. long each season, avellaneous-umbrinous within, mouths circular to slightly angular, $3-5$ to a mm., edges entire, white or cremeous, becoming umbrinous; spores ovoid, attenuate and truncate at the apex, yellowish-brown, 9-II $\times 5^{-8} \mu$; stipe usually eccentric or lateral, erect or ascending, equal or slightly enlarged above, cylindric, bay, laccate, the substance similar to the context and darker at the center, 5-10 $\times 2-3 \mathrm{~cm}$.

Found in the Tepeite Valley, Mexico, at an elevation of 2,000 m. 


\section{Ganoderma subincrustatum Murrill}

Pileus corky, circular to fan-shaped, plane above, $4-8 \times 6-8 \times$ I-2 cm.; surface glabrous, shallowly sulcate, slightly zonate, laccate, lustrous, yellowish-red to bay or dark-brown; margin cream-colored, sterile, finely tomentose, obtuse to subacute, undulate to lobed; context punky to soft-corky, slightly zonate, homogeneous, isabelline, slightly darker next to the tubes, fulvous with age, I cm. thick; tubes annual, 3-5 mm. long, avellaneous within, mouths circular to slightly angular, whitish-stuffed, 4 to a mm., edges obtuse, entire, stramineous, becoming thinner and very pale avellaneous; spores ellipsoid, dark-brown, $8 \times 4 \mu$; stipe central to lateral, cylindric, subequal, $\mathrm{I}-5 \mathrm{~cm}$. long, $\mathrm{I}-2 \mathrm{~cm}$. thick, resembling the pileus in surface and context, but almost black in color.

Common on dead wood throughout.

\section{GaNODERMa ARgILlaCeum Murrill}

Pileus corky, dimidiate to circular, plane above, usually convex below, 5-10 $\times 8-12 \times 2-4 \mathrm{~cm}$.; surface glabrous, rugose, not conspicuously marked, laccate but not lustrous, avellaneous to dullchestnut-colored; margin cream-colored, broad, subobtuse to acute, straight, entire; context punky, concentrically banded, isabelline above, fulvous below, 5-10 $\mathrm{mm}$. thick; tubes annual, 5-Io mm. long, avellaneous within, mouths angular, 3-4 to a mm., edges thin, subentire, white to avellaneous, reddish-brown when bruised; spores ovoid, dark-brown, I $2 \times 7 \mu$; stipe central to lateral, short, of ten wanting, smooth, glabrous, laccate, lustrous, bay to black, I-2 X I cm., substance corky, otherwise like that of the pileus.

Occasional on dead trunks in Mexico, Cuba, St. Croix, and Grenada.

\section{Ganoderma parvulum Murrill}

A very small sessile fungus, shining-bay above and honeyyellow below; pileus woody, nearly circular in outline, attached by a point, convex above, plane or convex below, thickest behind, $2 \times 2.5 \times$ I cm.; surface glabrous, laccate, azonate, slightly tubercular, very lightly marked with a few concentric furrows, margin acute; context soft-woody, pale-ochraceous, $0.5 \mathrm{~cm}$. thick, with dark, horny radiations from the point of attachment; tubes not stratified, $3 \mathrm{~mm}$. long, 5 to a $\mathrm{mm}$., umbrinous within, mouths polygonal, honey-yellow, edges entire, obtuse; spores subglobose, smooth, pale-yellowish-brown, $5 \times 4 \mu$.

Found once on decayed wood in Nicaragua. 


\section{IO. GANODERMa NITIDUM Murrill}

Pileus corky to woody, dimidiate or nearly circular, applanate, rarely ungulate or columnar by accretion with age, convex above, slightly concave below, $6-10 \times 8-16 \times 1.5-7 \mathrm{~cm}$.; surface glabrous, abundantly sulcate, laccate, shining, bay to brownish-black, flaky from the excess of resin exuded; margin ochraceous, subobtuse, undulate to lobed, the new growth of ten forming a broad lustrous band bordering the hymenium; context very thin, punky, bay, homogeneous, I $-3 \mathrm{~mm}$. thick; tubes stratified, very long and slender, $1.5-2.5 \mathrm{~cm}$., umbrinous to fuliginous within, mouths circular to slightly angular, stuffed with whitish mycelium when young, edges obtuse to very thin and uneven, white to umbrinous.

Frequent on dead trunks in Honduras.

\section{Ganoderma AREOlatum Murrill}

Pileus very large and thick, of light weight, dimidiate, sessile, convex above, plane to slightly convex below, $13 \times 20 \times 8 \mathrm{~cm}$.; surface azonate, glabrous, somewhat uneven, becoming areolate by the cracking of the thin cuticle, fuliginous-bay to blackish-bay, pallid in the cracks, margin acute, undulate or slightly lobed, finely tomentose, grayish-brown; context soft-corky, somewhat zonate, $\mathbf{I}-3 \mathrm{~cm}$. thick; tubes minute, corky to woody, isabelline within and without, not distinctly stratified, $3-5 \mathrm{~cm}$. long, mouths subangular to irregular, 2-3 to a mm., edges thin, entire; spores ellipsoid, smooth, hyaline or very pale yellowish, usually pointed at one end, copious, II $\times 5^{-6} \mu$.

Collected once on the dead trunk of a silk-cotton tree near Colima, Mexico.

\section{I2. Ganoderma tuberculosum Murrill}

Pileus rigid, dimidiate to reniform, applanate, 7-I5 $\times$ I2-30 $\times 2-4 \mathrm{~cm}$.; surface glabrous, laccate, shining, many times shallowly sulcate, radiate-rugose, roughly tuberculose, especially in large specimens, usually umbonate behind, bay to dull-brown with age; margin cremeous to fulvous, glabrous, slightly undulate, subobtuse to truncate with age; context punky to softcorky, isabelline above, fulvous below, castaneous with age, homogeneous, 5-10 $\mathrm{mm}$. thick; tubes perennial, indistinctly stratified, $\mathrm{I}-2.5 \mathrm{~cm}$. long, avellaneous within, slightly darker with age, mouths circular, 4 to a mm., not stuffed when young, edges obtuse, cream-colored, at length thin and darker in color; spores ovoid, dark-brown, $8 \times 6 \mu$.

Occasional on dead wood in British Honduras, Panama, western Jamaica, and Cuba. 


\section{I3. Ganoderma SEsSILIForme Murrill}

Pileus corky to woody, dimidiate, sessile or very slightly stipitate, slightly conchate to fan-shaped, thickest behind, thin at the margin, 3-5 $\times 6-9 \times 1.5^{-2} \mathrm{~cm}$.; surface laccate in places but mostly covered with powdery conidia, rugose, slightly concentrically sulcate, reddish-chestnut or dull-brownish, margin pallid, rather thin, sterile, slightly undulate; context corky, radiatefibrous, isabelline to isabelline-fulvous, thinner than the length of the tubes; tubes about $7 \mathrm{~mm}$. long, slender, 4-6 to a mm., avellaneous within; mouths circular, edges entire, rather thick, becoming thinner with age, pallid with a greenish-white tint; spores oblong-ovoid, pointed at one end, minutely roughened, thick-walled, umbrinous under a microscope, copious, Io-I I $\times 5 \mu$.

Collected once on dead wood near Cuernavaca, Mexico.

\section{CERRENA (Micheli) S. F. Gray}

Hymenophore small, epixylous, sessile, conchate, annual; surface anoderm, hairy or subglabrous, zonate or sulcate; context thin, white, fibrous, flexible; hymenium at first labyrinthiform, soon becoming irpiciform from the splitting of the dissepiments; spores smooth, hyaline.

\section{Cerrena stereoides (Fries) Murrill}

Pileus coriaceous, flexible, dimidiate or flabelliform, umbonateaffixed, applanate, $2 \times 2-3 \times 0.1-0.2 \mathrm{~cm}$.; surface pale-isabelline, concentrically lineate or slightly grooved, fibrillose to glabrous; margin very acute, straight, pallid, undulate or slightly lobed; context thin, white to pallid, membranous; tubes I-I.5 $\mathrm{mm}$. long, white to pallid within, soon becoming irpiciform, mouths I-2 to a mm., irregular, at first daedaleoid, soon splitting into sharp, slender, forked teeth, which are at first white or yellowish, becoming avellaneous-isabelline and glistening.

Found once on dead trunks at San José, Costa Rica.

\section{DAEDALEA Pers.}

Hymenophore epixylous, usually large and annual, sessile, applanate to ungulate; surface anoderm, glabrous, often zonate; context white or wood-colored, rigid, woody or punky; hymenium normally labyrinthiform, but varying to lamellate and porose in some species; spores smooth, hyaline.

Pileus 5-10 $\mathrm{cm}$. broad.

Furrows 5-10 mm. deep.

I. D. confragosa.

Furrows I-2 mm. deep.

2. D. favoloides. 
Pileus ro- $30 \mathrm{~cm}$. broad.

Furrows less than $0.5 \mathrm{~mm}$. in transverse diameter; surface white or yellowish.

Context white.

Context isabelline to umbrinous.

3. D. amanitoides.

4. D. microsticta.

Furrows 2-3 mm. in transverse diameter; surface brownish.

5. D. Sprucei.

\section{Daedalea confragosa (Bolt.) Pers.}

Pileus corky to woody, imbricate, sessile, dimidiate, convex or plane above, variable in size, $2-7 \times 3-10 \times 0.5-1.5 \mathrm{~cm}$.; surface multizonate, rugose, scrupose, often tuberculose, becoming glabrous, isabelline or avellaneous to latericeous-fuscous; margin thin, entire to lobed, pallid, fertile, dark-brown when bruised; context corky to woody, white to avellaneous, zonate, 3-1o $\mathrm{mm}$. thick; tubes very variable, porose or labyrinthiform, often becoming lamellate with age, $0.5^{-1.5} \mathrm{~mm}$. broad, 5-10 $\mathrm{mm}$. deep, white or avellaneous within, mouths grayish-pruinose when young, becoming umbrinous or reddish-fuscous, edges thin, becoming lacerate-dentate and often fimbriate, turning at once to yellowish-brown when bruised; spores smooth, hyaline, cylindric to ellipsoid, $5^{-8} \times 2-3 \mu$.

Found once on dead wood in Mexico.

\section{Daedalea favoloides Murrill}

Pileus coriaceous, reniform, applanate or subconchate, narrowly attached, 5-6 $\times 8$-10 $\times 0.3 \mathrm{~cm}$.; surface glabrous, somewhat rugose, narrowly, concentrically zonate-sulcate, avellaneous or avellaneous-umbrinous tinged with bay, margin thin, lobed; context very thin, punky, pallid to slightly yellowish-brown, about $2 \mathrm{~mm}$. thick; furrows $\mathrm{I}-2 \mathrm{~mm}$. deep, labyrinthiform, varying to circular or sublamellate in places, very narrow, about 2 to a $\mathrm{mm}$., avellaneous, becoming umbrinous with age, edges thin, dentate to subirpiciform.

Collected once on a fallen $\log$ in a moist virgin forest near Jalapa, Mexico. Very similar to Favolus tenuis above, but with the hymenium partly tubular and partly furrowed.

\section{Daedalea amanitoides Beauv.}

Pileus very thin and large, flexible, corky-woody, subsessile, usually attached by an orbicular peltate base, explanate, umbilicate, circular to fan-shaped, variable, $8-20 \times 15-30 \times 0.2-$ $0.5 \mathrm{~cm}$.; surface varying from entirely azonate to zonate, and from milk-white to partly avellaneous, glabrous, slightly sulcate, sometimes blackening behind with age; margin thin, white, 
undulate to lobed; context milk-white, floccose, homogeneous, 2-4 $\mathrm{mm}$. thick; tubes very variable in shape, circular to labyrinthiform and lamelloid, narrow, hardly exceeding $0.5 \mathrm{~mm}$. in breadth, 2-3 $\mathrm{mm}$. deep, white within, edges white to discolored, thin, uneven, dentate with age; spores oblong, smooth, hyaline, 5-8 $\times 2-3 \mu$.

Extremely common throughout on dead wood of various kinds.

\section{Daedalea microsticta Cooke}

Pileus corky, applanate, IO-I5 $\mathrm{cm}$. broad and $3 \mathrm{~cm}$. thick behind; surface glabrous, papillose, pale-wood-colored, margin acute, lineate-fuscous; context isabelline to umbrinous, punky to corky; hymenium varying from poroid to very narrowly labyrinthiform, the edges of the furrows being at first pallid and later becoming fuscous and lacerate.

Frequent on dead wood in Mexico and Central America. Described from Rio de Janeiro, Brazil.

\section{Daedalea Sprucei Berk.}

Pileus corky, flexible, very large, applanate, concave below, dimidiate to fan-shaped, thicker behind, 10-20 $\times$ I5-30 $\times$ I-2.5 $\mathrm{cm}$.; surface multizonate, slightly furrowed, finely tomentose to glabrous, rugose, sometimes tuberculose, dark-avellaneous or umbrinous, becoming blackish behind; margin thin, zonate, pallid; context zonate, soft-corky, isabelline, nearly fulvous when dry, 3-5 $\mathrm{mm}$. thick; tubes daedaleoid, becoming irpiciform, white within, unctuous to the touch, $2-3 \mathrm{~mm}$. broad, $\mathrm{I}-2 \mathrm{~cm}$. deep, edges thick, firm, soon splitting into flattened teeth, isabelline to fulvous or fuliginous; spores globose, smooth, brownish, $4-6 \mu$.

Occasional on dead trunks in Mexico, as well as in British Guiana and Brazil. This species or a closely related one has been found several times recently in Cuba, sometimes on living trunks.

\section{LENZITES Fries}

Hymenophore small, annual, epixylous, sessile, conchate; surface anoderm, usually zonate and tomentose; context white, coriaceous, flexible; hymenium lamellate, the radiating gill-like dissepiments connected transversely at times, especially in youth; spores smooth, hyaline. 
Surface of the pileus conspicuously tomentose.

Furrows $\mathrm{I}-2 \mathrm{~mm}$. broad, 3-10 mm. deep.

r. L. betulina.

Furrows $0.5-1 \mathrm{~mm}$. broad, 2-3 mm. deep.

Furrows frequently anastomosing; edges ochraceous.

Furrows rarely anastomosing; edges cremeous and much thinner.

2. L. betuliniformis.

3. L. subbetulina.

Surface of the pileus finely pubescent.

4. L. cubensis.

Surface of the pileus glabrous.

5. L. Earlei.

\section{Lenzites betulina (L.) Fries}

Pileus thin, coriaceous, sessile, dimidiate to flabelliform, imbricate, conchate, 3-4 $\times 4-7 \times 0.3-1 \mathrm{~cm}$.; surface conspicuously tomentose, velvety, multizonate, somewhat uneven, often radiate-rugose to plicate, avellaneous with latericeous zones, becoming olivaceous with age; margin thin, undulate to lobed at times; context very thin, white, membranous, scarcely a $\mathrm{mm}$. thick; furrows slightly anastomosing when very young, $\mathrm{I}-2 \mathrm{~mm}$. broad, 3-10 $\mathrm{mm}$. deep, edges thin, entire to undulate, slightly notched with age, cremeous within, ochroleucous to sordidochraceous without; spores globose, smooth, hyaline, $6 \mu$.

Occasional on dead deciduous wood at Jalapa and in the Tepeite Valley, Mexico.

\section{LENZITES BETULINIFORMIS Murrill}

Pileus thin, coriaceous to woody, sessile, dimidiate, imbricate, conchate, $2-3 \times 3-5 \times 0.3-0.5 \mathrm{~cm}$.; surface velvety-tomentose, narrowly multizonate, smooth, isabelline to avellaneous, some of the zones slightly darker; margin thin, fertile, rarely lobed; context white, membranous, scarcely a mm. thick; furrows ochraceous within, frequently anastomosing both in front and behind and interrupted by pores and bifurcations, $0.5^{-1} \mathrm{~mm}$. broad, $2-3 \mathrm{~mm}$. deep, edges thin, undulate to lacerate, often porose.

Found once on dead wood at Jalapa, Mexico.

\section{Lenzites subbetulina Murrill}

Pileus thin, coriaceous, flexible, reniform, narrowly attached, somewhat conchate, $2.5 \times 4 \times 0.3-0.6 \mathrm{~cm}$.; surface velvety, tomentose or finely hirsute, narrowly concentrically sulcatezonate, cremeous to greenish-avellaneous, the green color probably being due to algae, margin thin, entire or slightly undulate; context thin, white; hymenium cremeous, furrows very narrow, rarely anastomosing, about $0.5 \mathrm{~mm}$. broad and $1.5-2.5 \mathrm{~mm}$. deep; edges thin, undulate to slightly lacerate, coriaceous, cremeous within. 
Collected once on dead wood in a coffee plantation at Orizaba, Mexico.

\section{Lenzites cubensis Berk. \& Curt.}

Pileus rigid, corky to woody, dimidiate to flabelliform, applanate or convex, $4 \times 6 \times 0.3-0.5 \mathrm{~cm}$.; surface subvelvety, zonate, rugose, finely tomentose, isabelline; margin thin, ochraceous, fertile; context soft-corky to membranous, $\mathrm{x}-2 \mathrm{~mm}$. thick, isabelline in type specimens; furrows repeatedly forked, I $\mathrm{mm}$. broad, edges crenate or crisped, broad, concolorous.

Found once on dead wood in Cuba.

\section{Lenzites Earlei Murrill}

Pileus thin, dry, membranous, flexible, dimidiate, $3 \times 5 \times 0.5$ $\mathrm{cm}$.; surface glabrous, smooth, finely zonate, white; margin very thin, uneven, reddish-brown, multizonate, pellucid on the extreme border; context thin, white, scarcely a mm. thick; furrows not anastomosing at the base, very narrow and long, porose or forked at the margin, $0.3-0.5 \mathrm{~mm}$. broad, 2-5 mm. deep, edges white, crisped, very thin, lacerate-fimbriate with age.

Found once on a fence post at Port Antonio, Jamaica.

\section{5o. GLOEOPHYLLUM P. Karst.}

Hymenophore small, annual, epixylous, sessile; surface hairy or glabrous, anoderm, often zonate; context tough, brown; hymenium normally lamelloid or daedaleoid, but frequently poroid in some species; spores smooth, hyaline.

Hymenium lamelloid from the first; pileus very thin, dry, flexible.

I. G. striatum.

Hymenium not lamelloid from the first, but often becoming so with age; pileus thicker and usually rigid.

Context rosy-incarnate; furrows about $0.5 \mathrm{~mm}$. broad.

Context ferruginous to castaneous; furrows about $1 \mathrm{~mm}$. broad.

Surface hirsute.

Surface finely tomentose to glabrous.

2. G. trabeiforme.

\section{Gloeophyllum striatum (Sw.) Murrill}

Pileus membranous, flexible, dry, dimidiate to flabelliform, conchate, sessile, sometimes spuriously stipitate, imbricate, laterally connate and broadly attached or umbonate-affixed according to its position on the substratum, $2-6 \times 5^{-8} \times 0.3^{-}$ $0.5 \mathrm{~cm}$.; surface anoderm, distinctly tomentose, zonate, opaque, 
isabelline to umbrinous or cinereous; margin very thin, fertile, undulate, eroded with age; context very thin, punky, scarcely a $\mathrm{mm}$. thick in most specimens, umbrinous; tubes lamelloid from the first, avellaneous to umbrinous, furrows $I-1.5 \mathrm{~mm}$. broad, 2-4 mm. deep, edges thin, entire to irregularly notched and splitting with age, especially behind; spores oblong, smooth, hyaline, 6-8 $\times 3-4 \mu$.

Extremely common throughout on dead wood of various kinds.

\section{Gloeophyllum trabeiforme Murrill}

Pileus corky, flabelliform, narrowly attached by a stipitiform base, plane or slightly depressed above, convex below, 2.5-3.5 $\times$ $3-5.5 \times 0.5^{-1} \mathrm{~cm}$.; surface glabrous, slightly roughened with radiating raised lines resembling appressed fibers, somewhat shining, slightly zonate or sulcate, pale-fulvous with a chestnut tint, marked with two or more narrow, irregular, pale-chestnut zones, margin thin, entire, concolorous; context punky, thin, rosy-incarnate; furrows $0.5 \mathrm{~mm}$. broad, 2-4 mm. deep, fuliginouschestnut within and without; mouths irregular, varying from nearly circular to daedaleoid or radially elongate, frequently interrupted; edges thin, uneven or lacerate with age.

Collected once on dead wood near Xuchiles, Mexico.

\section{Gloeophyllum hirsutum (Schaeff.) Murrill}

Pileus hard, corky to woody, slightly flexible, imbricate, sessile, laterally connate, often decurrent, oblong-dimidiate to flabelliform, conchate, $2-3 \times 4-8 \times 0.3-1 \mathrm{~cm}$.; surface zonate, strigosetomentose, anoderm, rather uneven, reddish-fulvous to fuliginous or umbrinous; margin rather thick, sterile, isabelline, undulate, finely tomentose, becoming acute and darker in age; context soft-corky, homogeneous, fulvous, about $2 \mathrm{~mm}$. thick; tubes usually lamelloid, anastomosing when young, ochraceous to grayish-umbrinous, $0.5-1 \mathrm{~mm}$. broad, 2-5 $\mathrm{mm}$. deep, edges thin, undulate; in a poroid variety, tubes circular, regular, 2 to a mm., edges thick, firm, entire; spores ellipsoid, smooth, hyaline, 8-12 $\times 3-4 \mu$.

Occasional in southern Florida, Jamaica, and Mexico on dead wood. More frequent in the mountains.

\section{Gloeophyllum Berkeleyi (Sacc.) Murrill}

Pileus corky, subrigid, dimidiate, sessile, imbricate, laterally connate, plane above, usually convex below, $3^{-5} \times 5^{-10} \times 0.8-2$ $\mathrm{cm}$.; surface finely tomentose to glabrous and subshining, zonate, 
slightly sulcate, radiately furrowed, cremeous or isabelline to fulvous or chestnut-colored and finally black behind; margin obtuse, tomentose, white or cream-colored to ochraceous-fulvous; context punky to soft-corky, homogeneous, 2-4 mm. thick, fulvous, tinged with rhubarb when very young; tubes irregular, daedaleoid, branched or forked, becoming much elongate radially, 5-I5 mm. deep, about I mm. broad, wider with age, edges white or ochraceous to fulvous, thin, rigid, sinuate, rarely splitting even with age.

Frequent in southern Florida, the Bahamas, Cuba, Jamaica, Mexico, and certain other parts of tropical America on pine railway ties and other forms of dead coniferous wood. 


\section{INDEX TO GENERA WITH SPECIES}

Abortiporus, 50

distortus (Schw.) Murrill, 50

tropicalis Murrill, 50

Amauroderma, 9I avellaneum Murrill, 94

Brittonii Murrill, 95

Chaperi (Pat.) Murrill, 94 coffeatum (Berk.) Murrill, 93

flaviporum Murrill, 94

nutans (Fries) Murrill, 92

regulicolor (Cooke) Murrill, 93

renatum (Berk.) Murrill, 92

subrenatum Murrill, 93

\section{Bjerkandera, 25}

adusta (Willd.) P. Karst., 26

albostygia (Berk. \& Curt.) Murrill, 26

subsimulans Murrill, 27

terebrans (Berk. \& Curt.) Murrill, 26

Cerrena, I0I

stereoides (Fries) Murrill, Ior

Cerrenella, 53

farinacea (Fries) Murrill, 54

Ravenelii (Berk.) Murrill, 54

subcoriacea Murrill, 54

Coltricia, 72

cinnamomea (Jacq.) Murrill, 72

focicola (Berk. \& Curt.) Murrill, 73

perennis (L.) Murrill, 72

spathulata (Hook.) Murrill, 73

Coltriciella, $7 I$

dependens (Berk. \& Curt.) Murrill, $7 I$

Coriolellus, I 7

Sepium (Berk.) Murrill, I7

Coriolopsis, 54

caperata (Berk.) Murrill, 60

caperatiformis Murrill, 59

cirrifer (Berk. \& Curt.) Murrill, 57

crocata (Fries) Murrill, 55

crocatiformis Murrill, 57

fulvocinerea Murrill, 56

fumosa Murrill, 58

nigrocinerea Murrill, 59

occidentalis (Klotzsch) Murrill, 56

rigida (Berk. \& Mont.) Murrill, 56

sarcitiformis Murrill, 58

subglabrescens Murrill, 60

Taylori Murrill, 57

vibratilis (Berk. \& Curt.) Murrill, 59 vittata (Ellis \& Macbr.) Murrill, 59

Coriolus, 5
Coriolus abietinus (Dicks.) Quél., I 7 armenicolor (Berk. \& Curt.) Pat., Io brachypus (Lév.) Murrill, to concavus Murrill, 1o cyphelloides (Fries) Murrill, I6 delectans Murrill, 9

depauperatus (Pat.) Murrill, 8 effusus Murrill, I5

fulvo-umbrinus Murrill, I3

haedinus (Berk.) Pat., II

Hollickii Murrill, I2

hondurensis Murrill, II

irpiciformis Murrill, I6 leiodermus (Mont.) Murrill, I3 maximus (Mont.) Murrill, I6 membranaceus (Sw.) Pat., I3 nigromarginatus (Schw.) Murrill, I3 ochrotinctellus Murrill, I2 orizabensis Murrill, 15 pallidofulvellus Murrill, 9 pavonius (Hook.) Murrill, I5 pertenuis Murrill, I2 pinsitus (Fries) Pat., I4 prolificans (Fries) Murrill, I7 scutatus Murrill, I4 sector (Ehrenb.) Pat., 8 sericeohirsutus (Klotzsch) Murrill, I4 sobrius (Berk. \& Curt.) Murrill, I6 subpavonius Murrill, 8 substipitatus Murrill, II tepeitensis Murrill, 9 versicolor (L.) Quél., 7 xuchilensis Murrill, 9

Cryptoporus, 73 volvatus (Peck) Hubbard, 74

Cycloporellus, 66 iodinus (Mont.) Murrill, 66

Daedalea, Ior amanitoides Beauv., I02 confragosa (Bolt.) Pers., I02 favoloides Murrill, 102 microsticta Cooke, IO3 Sprucei Berk., I03

Earliella, 30 corrugata (Pers.) Murrill, 30

Elf vingia, 90 Lionnetii (Rolland) Murrill, 9I tornata (Pers.) Murrill, gI

Elf vingiella, 90 fasciata (Sw.) Murrill, 90

Favolus, 63 
Favolus tenuiformis Murrill, 64 tenuis (Hook.) Murrill, 64 variegatus (Berk.) Murrill, 64

Flaviporellus, 52 Splitgerberi (Mont.) Murrill, 52

Flaviporus, 65 crocitinctus (Berk. \& Curt.) Murrill, 65 rufoflavus (Berk. \& Curt.) Murrill, 65

Fomes, 74

annosus (Fries) Cooke, 76

Auberianus (Mont.) Murrill, 77

geotropus Cooke, 78

ligneus (Berk.) Cooke, 76

roseus (Alb. \& Schw.) Cooke, 75

rubritinctus Murrill, 77

Sagraeanus (Mont.) Murrill, 75

scutellatus (Schw.) Cooke, 76

subferreus Murrill, 76

turbinatus (Pat.) Murrill, 75

ungulatus (Schaeff.) Sacc., 77

Fomitella, 78

fumoso-avellanea (Romell) Murrill, 78

supina (Sw.) Murrill, 78

Fulvifomes, $8 \mathrm{I}$

calcitratus (Berk. \& Curt.) Murrill, 84

Cedrelae Murrill, 88

cinchonensis Murrill, 87

dependens Murrill, 88

extensus (Lév.) Murrill, 86

grenadensis Murrill, 83

hydrophilus Murrill, 86

jamaicensis Murrill, 83

linteus (Berk. \& Curt.) Murrill, 84

melleicinctus Murrill, 85

pseudosenex Murrill, 83

sarcitus (Fries) Murrill, 85

sublinteus Murrill, 86

subpectinatus Murrill, 84

Swieteniae Murrill, 87

troyanus Murrill, 87

Underwoodii Murrill, 88

yucatanensis Murrill, 85

Funalia, 6o

aculeifer (Berk. \& Curt.) Murrill, 62 cladotricha (Berk. \& Curt.) Murrill, 6 I

hispidula (Berk. \& Curt.) Murrill, 6I versatilis (Berk.) Murrill, 6I

Ganoderma, 95

areolatum Murrill, 100 argillaceum Murrill, 99

Curtisii (Berk.) Murrill, 98

nitidum Murrill, roo

parvulum Murrill, 99

perzonatum Murrill, 96

praelongum Murrill, 96

pulverulentum Murrill, 97

sessiliforme Murrill, I or
Ganoderma stipitatum Murrill, 98 subfornicatum Murrill, 97 subincrustatum Murrill, 99 tuberculosum Murrill, I00

Gloeophyllum, I05 Berkeleyi (Sacc.) Murrill, I06 hirsutum (Schaeff.) Murrill, I06 striatum (Sw.) Murrill, I05 trabeiforme Murrill, 106

Grifola, 5 I lentifrondosa Murrill, 5I

Hapalopilus, 62 gilvus (Schw.) Murrill, 63 licnoides (Mont.) Murrill, 63

Hexagona, 33 brunneola (Berk. \& Curt.) Murrill, 39 caperata (Pat.) Murrill, 37 cucullata (Mont.) Murrill, 39 daedalea (Link) Murrill, 35 daedaleiformis Murrill, 35 floridana Murrill, 34 fragilis Murrill, 36 hondurensis Murrill, 38 indurata (Berk.) Murrill, 39 Maxoni Murrill, 34 motzorongensis Murrill, 37 portoricensis Murrill, 38 princeps (Berk. \& Curt.) Murrill, 34 pseudoprinceps Murrill, 34 purpurascens (Berk. \& Curt.) Murrill, 38

reniformis Murrill, 36

subcaperata Murrill, 36

subpurpurascens Murrill, 38

sulfurea Murrill, 37

tessellatula Murrill, 35

Inonotus, 67

corrosus Murrill, 69

fruticum (Berk. \& Curt.) Murrill, 7o

fulvomelleus Murrill, 68

jamaicensis Murrill, 68

leprosus (Fries) Murrill, 69

pertenuis Murrill, 67

porrectus Murrill, 68

pusillus Murrill, 70

radiatus (Sow.) P. Karst., 70

Wilsonii Murrill, 69

Irpiciporus, 4 cubensis (Berk. \& Curt.) Murrill, 5 lacteus (Fries) Murrill, 5

Laetiporus, $\mathbf{5 2}^{2}$ speciosus (Batt.) Murrill, 52

Lenzites, 103 betulina (L.) Fries, I04 betuliniformis Murrill, 104 cubensis Berk. \& Curt., I05 Earlei Murrill, I05 subbetulina Murrill, I04 
Microporellus, 39

dealbatus (Berk. \& Curt.) Murrill, 40 holotephrus (Berk. \& Curt.) Murrill, 40 porphyritis (Berk.) Murrill, 40 unguicularis (Fries) Murrill, 40

Nigrofomes, 89 melanoporus (Mont.) Murrill, 89

Nigroporus, 66 vinosus (Berk.) Murrill, 66

Phaeolopsis, 53

Verae-crucis (Berk.) Murrill, 53

Phaeolus, 70 sistotremoides (Alb. \& Schw.) Murrill, 7 I

Pogonomyces, 65 hydnoides (Sw.) Murrill, 65

Polyporus, 4I

Acicula Berk. \& Curt., 44 aemulans Berk. \& Curt., 45 arcularius (Batsch) Fries, 45 Blanchetianus Berk. \& Mont., 49 Cowellii Murrill, 46 cyathiformis Lév., 44 diabolicus Berk., 48 discoideus Berk. \& Curt., 44 elegans (Bull.) Fries, 47 hydniceps Berk. \& Curt., 42 maculosus Murrill, 46 marasmioides (Pat.) Murrill, 47

Marbleae Murrill, 48 obolus Ellis \& Macbr., 44 praeguttulatus Murrill, 49 scabellus (Pat.) Murrill, 49 scabriceps Berk. \& Curt., 43 subelegans Murrill, 48 Tricholoma Mont., 46 Tuba Berk. \& Curt., 43 variiporus Murrill, 45 virgatus Berk. \& Curt., 43 Wrightii Murrill, 47

Porodaedalea, 89 Pini (Thore) Murrill, 89

Porodisculus, 32 pendulus (Schw.) Murrill, 32

Pycnoporus, 5I sanguineus (L.) Murrill, 5I

Pyropolyporus, 79 albomarginatus (Lév.) Murrill, 79 Baccharidis (Pat.) Murrill, 80 Calkinsii Murrill, 8 I Haematoxyli Murrill, 80 inflexibilis (Berk.) Murrill, 81
Pyropolyporus Robinsoniae Murrill, 80 roseocinereus Murrill, 8I

Rigidoporus, 30 contrarius (Cooke) Murrill, 32 evolutus (Berk. \& Curt.) Murrill, 3 I Liebmanni (Fries) Murrill, 3 I microstomus (Berk. \& Curt.) Murrill, 31 substereinus Murrill, 3 I surinamensis (Miq.) Murrill, 32

Spongipellis, $24^{\prime}$ hydrophilus (Berk. \& Curt.) Murrill, 25

luridescens Murrill, 25

substuppeus (Berk. \& Curt.) Murrill, 25

Spongiporus, 18 altocedronensis Murrill, 18

Tomophagus, 18 Colossus (Fries) Murrill, 18

Trametes, 27 cubensis (Mont.) Sacc., 28 havannensis (Berk. \& Curt.) Murrill, 28

jalapensis Murrill, 29

jamaicensis Murrill, 28

lignea Murrill, 29

nivosa (Berk.) Murrill, 27

rutilantiformis Murrill, 29

submurina Murrill, 28

subscutellatus Murrill, 29

Trichaptum, 62

trichomallum (Berk. \& Mont.) Murrill, 62

Tyromyces, I9

albogilvus (Berk. \& Curt.) Murrill, 24 caesius (Schrad.) Murrill, 22

cinchonensis Murrill, 23

duracinus (Pat.) Murrill, 24

fulvitinctus (Berk. \& Curt.) Murrill, 24

graminicola Murrill, 2 I

lacteus (Fries) Murrill, 23

leucomallus (Berk. \& Curt.) Murrill, 23

nivosellus Murrill, 20

Palmarum Murrill, 20

palustris (Berk. \& Curt.) Murrill, 20 semipileatus (Peck) Murrill, 22 semisupiniformis Murrill, 22

Smallii Murrill, 2 I versicutis (Berk. \& Curt.) Murrill, 2 I 


\section{INDEX TO SPECIES}

adusta (Bjerkandera), 26

abietinus (Coriolus), 17

Acicula (Polyporus), 44

aculeifer (Funalia), 62

aemulans (Polyporus), 45

albogilvus (Tyromyces), 24

albomarginatus (Pyropolyporus), 79

albostygia (Bjerkandera), 26

altocedronensis (Spongiporus), I 8

amanitoides (Daedalea), 102

annosus (Fomes), 76

arcularius (Polyporus), 45

areolatum (Ganoderma), roo

argillaceum (Ganoderma), 99

armenicolor (Coriolus), ro

Auberianus (Fomes), 77

avellaneum (Amauroderma), 94

Baccharidis (Pyropolyporus), 80

Berkeleyi (Gloeophyllum), ro6

betulina (Lenzites), 104

betuliniformis (Lenzites), 104

Blanchetianus (Polyporus), 49

brachypus (Coriolus), ro

Brittonii (Amauroderma), 95

brunneola (Hexagona), 39

caesius (Tyromyces), 22

calcitratus (Fulvifomes), 84

Calkinsii (Pyropolyporus), 8I

caperata (Coriolopsis), 60

caperata (Hexagona), 37

caperatiformis (Coriolopsis), 59

Cedrelae (Fulvifomes), 88

Chaperi (Amauroderma), 94

cinchonensis (Fulvifomes), 87

cinchonensis (Tyromyces), 23

cinnabarinus (Pycnoporus), 52

cinnamomea (Coltricia), 72

cirrifer (Coriolopsis), 57

cladotricha (Funalia), $6 \mathrm{I}$

coffeatum (Amauroderma), 93

Colossus (Tomophagus), I 8

concavus (Coriolus), Io

confragosa (Daedalea), I02

contrarius (Rigidoporus), 32

corrosus (Inonotus), 69

corrugata (Earliella), 30

Cowellii (Polyporus), 46

craterellus (Polyporus), 44

crocata (Coriolopsis), 55

crocatiformis (Coriolopsis), 57

crocitinctus (Flaviporus), 65

cubensis (Irpiciporus), 5

cubensis (Lenzites), 105

cubensis (Trametes), 28 cucullata (Hexagona), 39

Curtisii (Ganoderma), 98

cyathiformis (Polyporus), 44

cyphelloides (Coriolus), I6

daedalea (Hexagona), 35

daedaleiformis (Hexagona), 35

dealbatus (Microporellus), 40

delectans (Coriolus), 9

depauperatus (Coriolus), 8

dependens (Coltriciella), 7 I

dependens (Fulvifomes), 88

diabolicus (Polyporus), 48

dictyoporus (Leucoporus), 45

discoideus (Polyporus), 44

distortus (Abortiporus), 50

dryophila (Fomitiporia), 8I

duracinus (Tyromyces), 24

Earlei (Lenzites), I05

effusus (Coriolus), 15

elegans (Polyporus), 47

evolutus (Rigidoporus), 3 I

extensus (Fulvifomes), 86

farinacea (Cerrenella), 54

fasciata (Elfvingiella), 90

favoloides (Daedalea), ro2

flaviporum (Amauroderma), 94

floridana (Hexagona), 34

focicola (Coltricia), 73

fragilis (Hexagona), 36

fruticum (Inonotus), 70

fulvitinctus (Tyromyces), 24

fulvocinerea (Coriolopsis), 56

fulvomelleus (Inonotus), 68

fulvo-umbrinus (Coriolus), 13

fumosa (Coriolopsis), 58

fumoso-avellanea (Fomitella), 78

geotropus (Fomes), 78

gilvus (Hapalopilus), 63

graminicola (Tyromyces), 21

grenadensis (Fulvifomes), 83

haedinus (Coriolus), II

Haematoxyli (Pyropolyporus), 80

havannensis (Trametes), 28

hirsutum (Gloeophyllum), 106

hispidula (Funalia), 6r

Hollickii (Coriolus), 12

holotephrus (Microporellus), 40

hondurensis (Coriolus), II

hondurensis (Hexagona), 38

hydniceps (Polyporus), 42

hydnoides (Pogonomyces), 65

hydrophilus (Fulvifomes), 86

hydrophilus (Spongipellis), 25

indurata (Hexagona), 39 
inflexibilis (Pyropolyporus), 81 iodinus (Cycloporellus), 66 irpiciformis (Coriolus), 16 jalapensis (Trametes), 29 jamaicensis (Fulvifomes), 83 jamaicensis (Inonotus), 68 jamaicensis (Trametes), 28 lacteus (Irpiciporus), 5 lacteus (Tyromyces), 23 lateritius (Fomes), 79 leiodermus (Coriolus), I3 lentifrondosa (Grifola), 5 I leprosus (Inonotus), 69 leucomallus (Tyromyces), 23 licnoides (Hapalopilus), 63 Liebmanni (Rigidoporus), 31 lignea (Trametes), 29 ligneus (Fomes), 76 linteus (Fulvifomes), 84 Lionnetii (Elfvingia), 9I luridescens (Spongipellis), 25 maculosus (Polyporus), 46 marasmioides (Polyporus), 47 Marbleae (Polyporus), 48 maximus (Coriolus), I6 Maxoni (Hexagona), 34 melanoporus (Nigrofomes), 89 melleicinctus (Fulvifomes), 85 membranaceus (Coriolus), I3 microsticta (Daedalea), I03 microstomus (Rigidoporus), 3 I motzorongensis (Hexagona), 37 mutabilis (Polyporus), 40 nigrocinerea (Coriolopsis), 59 nigromarginatus (Coriolus), 13 nitidum (Ganoderma), 100 nivosa (Trametes), 27 nivosellus (Tyromyces), 20 nutans (Amauroderma), 92 obolus (Polyporus), 44 occidentalis (Coriolopsis), 56 ochrotinctellus (Coriolus), 12 ohiensis (Fomes), 75 orizabensis (Coriolus), I5 pallidofulvellus (Coriolus), 9 Palmarum (Tyromyces), 20 palustris (Tyromyces), 20 parvulum (Ganoderma), 99 pavonius (Coriolus), 15 pendulus (Porodisculus), 32 perennis (Coltricia), 72 pertenuis (Coriolus), 12 pertenuis (Inonotus), 67 perzonatum (Ganoderma), 96 Pini (Porodaedalea), 89 pinsitus (Coriolus), 14, $6 \mathrm{I}$ porphyritis (Microporellus), 40 porrectus (Inonotus), 68 portoricensis (Hexagona), 38 praeguttulatus (Polyporus), 49 praelongum (Ganoderma), 96 princeps (Hexagona), 34 prolificans (Coriolus), I7 pseudoprinceps (Hexagona), 34 pseudosenex (Fulvifomes), 83 pulverulentum (Ganoderma), 97 purpurascens (Hexagona), 38 pusillus (Inonotus), 70 radiatus (Inonotus), 70 Ravenelii (Cerrenella), 54 regulicolor (Amauroderma), 93 renatum (Amauroderma), 92 reniformis (Hexagona), 36 rigida (Coriolopsis), 56 Robinsoniae (Pyropolyporus), 80 roseocinereus (Pyropolyporus), $8 \mathrm{I}$ roseus (Fomes), 75 rubritinctus (Fomes), 77 rufoflavus (Flaviporus), 65 rutilantiformis (Trametes), 29 Sagraeanus (Fomes), 75 sanguineus (Pycnoporus), 51 sarcitiformis (Coriolopsis), $5^{8}$ sarcitus (Fulvifomes), 85 scabellus (Polyporus), 49 scabriceps (Polyporus), 43 scutatus (Coriolus), I4 scutellatus (Fomes), 76 sector (Coriolus), 8 semipileatus (Tyromyces), 22 semisupiniformis (Tyromyces), 22 Sepium (Coriolellus), I7 sericeohirsutus (Coriolus), I4 sessiliforme (Ganoderma), I or sistotremoides (Phaeolus), 7 I Smallii (Tyromyces), 2 I sobrius (Coriolus), I6 spathulata (Coltricia), 73 speciosus (Laetiporus), 52 Splitgerberi (Flaviporellus), 52 Sprucei (Daedalea), I03 stereoides (Cerrena), I or stipitatum (Ganoderma), 98 striatum (Gloeophyllum), 105 subbetulina (Lenzites), 104 subcaperata (Hexagona), 36 subcoriacea (Cerrenella), 54 subelegans (Polyporus), 48 subferreus (Fomes), 76 subfornicatum (Ganoderma), 97 subglabrescens (Coriolopsis), 60 subincrustatum (Ganoderma), 99 sublinteus (Fulvifomes), 86 submurina (Trametes), 28 subpavonius (Coriolus), 8 subpectinatus (Fulvifomes), 84 subpurpurascens (Hexagona), $3^{8}$ subrenatum (Amauroderma), 93 subscutellatus (Trametes), 29 subsimulans (Bjerkandera), 26 substereinus (Rigidoporus), 3 I substipitatus (Coriolus), I I substuppeus (Spongipellis), 25 sulfurea (Hexagona), 37 
supina (Fomitella), 78

surinamensis (Rigidoporus), 32

Swieteniae (Fulvifomes), 87

Taylori (Coriolopsis), 57

tenuiformis (Favolus), 64

tenuis (Favolus), 64

tepeitensis (Coriolus), 9

terebrans (Bjerkandera), 26

tessellatula (Hexagona), 35

tornata (Elfvingia), 91

trabeiforme (Gloeophyllum), 106

Tricholoma (Polyporus), 46

trichomallum (Trichaptum), 62

tropicalis (Abortiporus), 50

troyanus (Fulvifomes), 87

Tuba (Polyporus), 43

tuberculosum (Ganoderma), 100

turbinatus (Fomes), 75

Underwoodii (Fulvifomes), 88 unguicularis (Microporellus), 40

ungulatus (Fomes), 77

variegatus (Favolus), 64

variiporus (Polyporus), 45

Verae-crucis (Phaeolopsis), 53

versatilis (Funalia), 6I

versicolor (Coriolus), 7

versicutis (Tyromyces), 2 I

vibratilis (Coriolopsis), 59

villosa (Funalia), $6 \mathrm{I}$

vinosus (Nigroporus), 66

virgatus (Polyporus), 43

vittata (Coriolopsis), 59

volvatus (Cryptoporus), 74

Wilsonii (Inonotus), 69

Wrightii (Polyporus), 47

xuchilensis (Coriolus), 9

yucatanensis (Fulvifomes), 85 


\section{Manuals of Polypores and Boletes}

By William A. Murrill, A.M., Ph.D., Assistant Director of the New York Botanical Garden, Editor of Mycologia, and Associate Editor of North American Flora.

Northern Polypores, issued December 8, 1914 . \$1.00 Including species found in Canada and the United States south to Virginia and west to the Rockies.

Southern Polypores, issued January 30, 1915 \$ \$1.00

Including species found in the United States from

North Carolina to Florida and west to Texas.

Western Polypores, issued March 25, 1915 . . .\$1.00

Including species found in the states on the Pacific coast from California to Alaska.

Tropical Polypores, issued in June, I9I5 . . . \$1.50

Including species found in Mexico, Central America, southern Florida, the West Indies, and other islands between North America and South America.

American Boletes, issued December 8, 1914 . . \$1.00

Including all the species found in temperate and tropical North America, both on the mainland and on the islands, south to South America.

The above prices include prepaid postage, even to foreign countries. No reduction is made to any one, dealers included. The author regrets that, owing to the small editions, no copies can be distributed for examination, but a free desk copy will be supplied, if requested, with an order for ten copies of the same book sent to one address.

Remit by Postoffice or Express Money Order, or, if by Check, please add Exchange.

\section{W. A. MURRILL}

Bronxwood Park

NEW YORK CITY 



UNIVERSITY OF CALIFORNIA LIBRARY

Los Angeles

This book is DUE on the last date stamped below. 
UC SOUTHERN REGIONAL LIBRARY FACILITY

AA 0009272220

SOUTHERN BRANCH, UNIVERSITY OF CALIFORNIA. LIBRARY,

LOS ANGELES, ĆALAr, 
